\title{
Confocal Imaging of Calcium Signal and Exocytosis at Individual Hair Cell Synapses
}

\author{
PhD Thesis \\ in partial fulfilment of the requirements \\ for the degree "Dr. rer. nat." \\ in the Neuroscience Program \\ at the Georg August University Göttingen, \\ Faculty of Biology
}

submitted by

Aaron Benson Wong

born in

Hong Kong

Göttingen, 2013 


\section{Declaration}

I hereby declare that this thesis has been written independently and with no other sources and aids than quoted.

Aaron Benson Wong

Göttingen, $28^{\text {th }}$ March, 2013 


\section{Table of Contents}

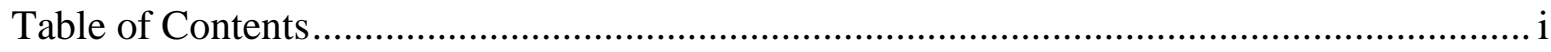

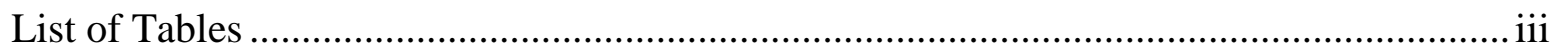

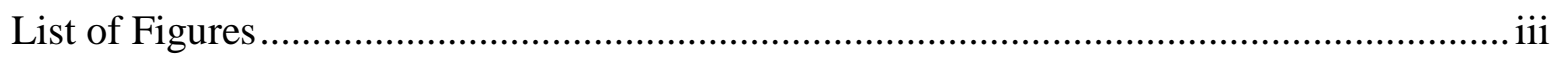

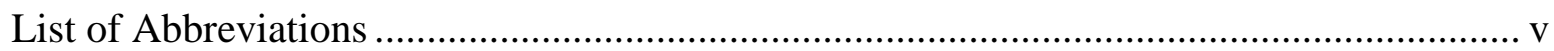

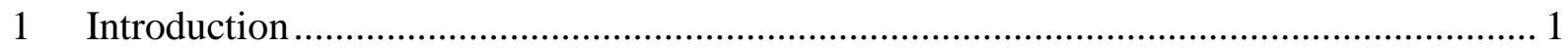

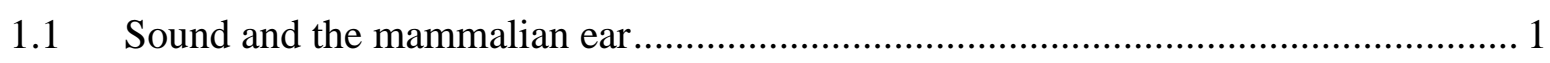

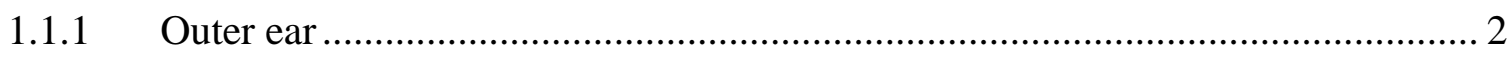

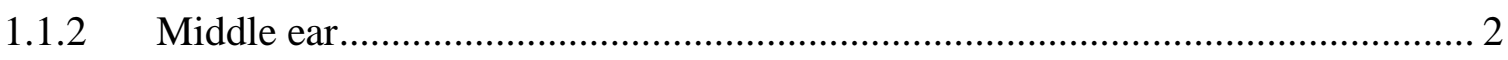

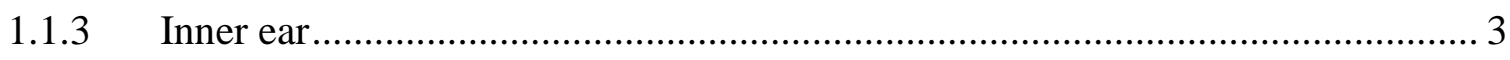

1.1.4 Sensory transduction at the organ of Corti ..................................................... 5



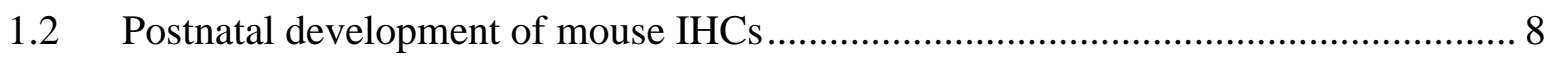

1.3 Diversity of spiral ganglion neuron (SGN) response and possible mechanisms ........ 9

1.3.1 Postsynaptic and efferent mechanisms.................................................... 10

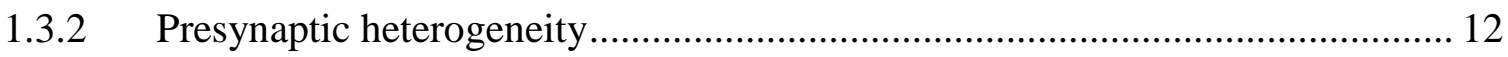

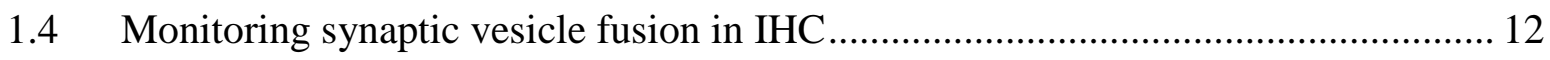

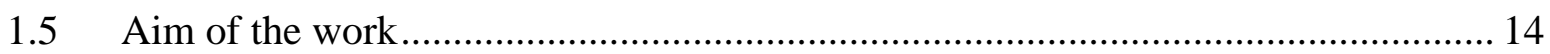

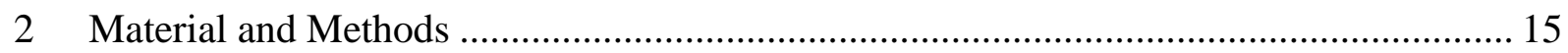

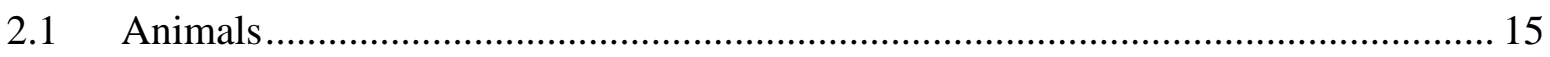

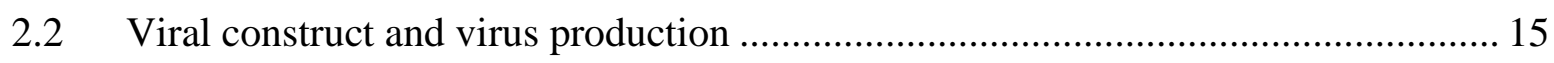

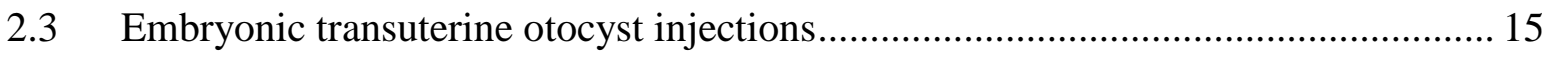

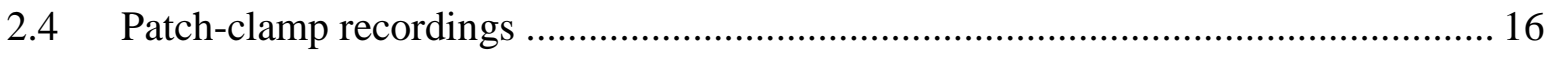

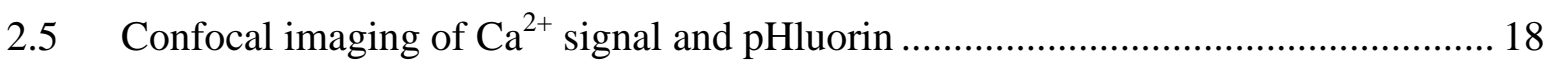

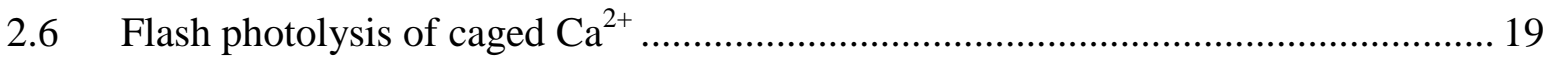

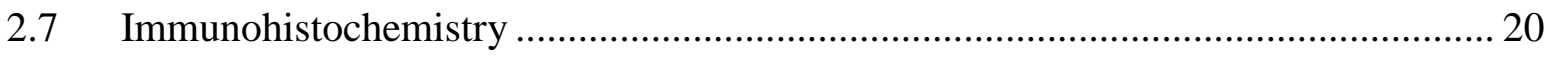

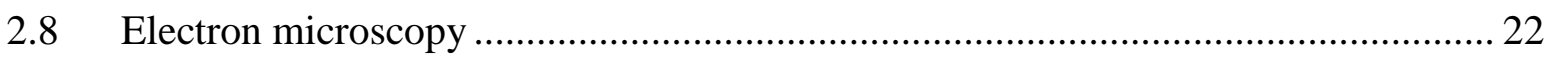

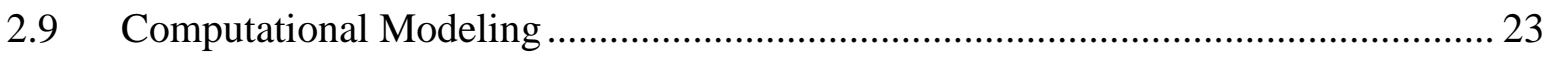

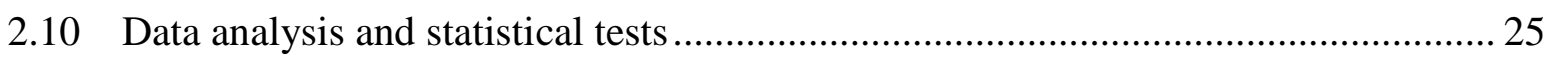



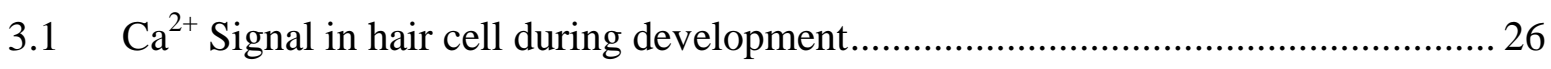

3.1.1 Naturalistic electrical activities and underlying calcium signals ....................... 26

3.1.2 Biophysical properties of calcium current....................................................... 34

3.1.3 Subcellular distribution of calcium channels .................................................. 36

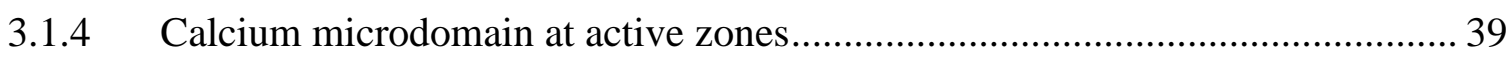

3.1.5 Concomitant changes in synaptic morphology ............................................. 44 
3.2 $\mathrm{Ca}^{2+}$ dependence of exocytosis in developing IHC ............................................. 48

3.2.1 Probing intrinsic $\mathrm{Ca}^{2+}$-dependence of exocytosis through $\mathrm{Ca}^{2+}$ uncaging ......... 48

3.2.2 Mathematical analysis of binding kinetics ....................................................... 51

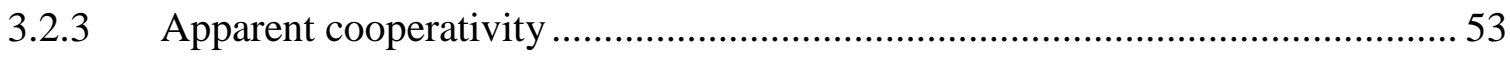

3.3 Characterization of vglut $1-\mathrm{pHluorin}(\mathrm{vGpH})$ Reporter in IHCs.............................. 55

3.3.1 Transduction efficiency, expression level and subcellular localization ............. 55

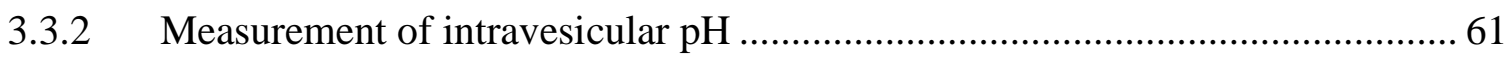



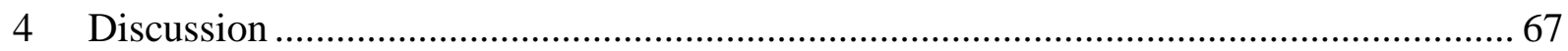

4.1 Release machinery of IHC ribbon synapse during postnatal development ............... 67

4.1.1 Developmental changes of in flash responses ............................................... 67

4.1.2 $\mathrm{Ca}^{2+}$ dependence of release in IHC and molecular candidates for $\mathrm{Ca}^{2+}$ sensor.. 70

4.2 From microdomain to nanodomain control of vesicle fusion................................... 71

4.2.1 Developmental decrease in apparent $\mathrm{Ca}^{2+}$ cooperativity of release ................... 71

4.2.2 Possible mechanisms for switch from microdomain to nanodomain................. 74

4.3 Refinement of synaptic scaffold and postsynaptic density during development....... 76

4.4 The synaptic $\mathrm{Ca}^{2+}$ signal and its developmental increase in heterogeneity ............... 77

4.4.1 AP-associated $\mathrm{Ca}^{2+}$ transients and their potential functions ............................. 77

4.4.2 Ability of mature synaptic $\mathrm{Ca}^{2+}$ signaling to follow high frequency ................. 78

4.4.3 Quantitative measurement of synaptic $\mathrm{Ca}^{2+}$ signal and possible errors ............. 81

4.4.4 Presynaptic heterogeneity as a mechanism to decompose auditory information 82

4.4.5 Possible mechanisms mediating presynaptic heterogeneity ............................. 84

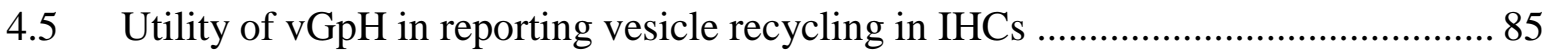

4.5.1 Extensive subcellular localization of $\mathrm{vGpH}$ in transduced IHCs ....................... 85

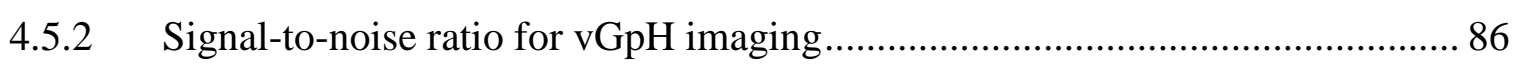

4.5.3 Alternative optical reporters for single AZ release ........................................ 86

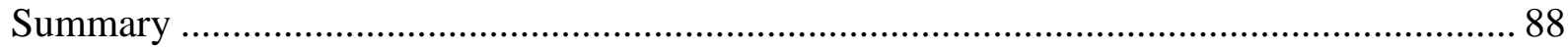

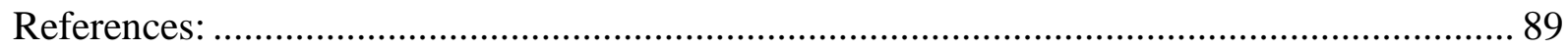

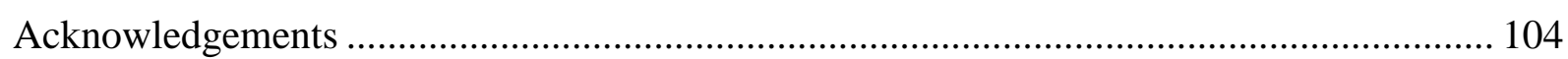

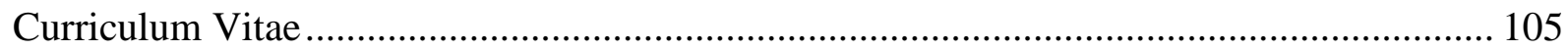

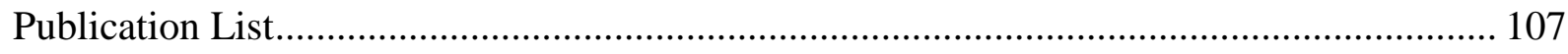




\section{List of Tables}

Table 1: Cutoff frequency of IHC voltage response to mechanotransduction-like current ..... 32

Table 2: Quantification of ultrastructural properties of developing IHC ribbon synapses 48

\section{List of Figures}

Figure 1.1: Gross anatomy of a human ear. 2

Figure 1.2: Detailed anatomy of the cochlea and the organ of Corti..................................... 3

Figure 1.3: Frequency separation by the cochlea............................................................ 4

Figure 1.4: Schematic illustration of a pre-hearing and a mature IHC . ................................ 9

Figure 1.5: Diverse SGN response properties and examples of possible mechanisms ............ 10

Figure 1.6: Use of pHluorin to monitor vesicle recycling ...................................................... 13

Figure 3.1: $\mathrm{Ca}^{2+}$ signal in IHC of prehearing animal. ......................................................... 27

Figure 3.2: Current template resembling transduction current............................................. 28

Figure 3.3: Response of IHCs to current injections mimicking natural mechano-electrical

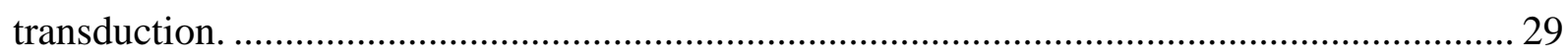

Figure 3.4: Peak-to-peak and steady-state depolarization of IHC voltage responses ............. 31

Figure 3.5: Synaptic calcium signal of mature IHCs with oscillating potential...................... 33

Figure 3.6: Unaltered biophysical properties of $\mathrm{Ca}^{2+}$ current during development. ................ 35

Figure 3.7: Non-stationary fluctuation analysis reveals channel number as major change in development 35

Figure 3.8: Maturational confinement of $\mathrm{Ca}^{2+}$ channel and $\mathrm{Ca}^{2+}$ signal towards active zone revealed by $\mathrm{Ca}^{2+}$ imaging and immunohistochemistry. 37

Figure 3.9: Estimation of density of membrane-associated vs ribbon-associated Cav1.3 ....... 39

Figure 3.10: Developmental emergence of ribbon-occupied AZs with intense $\mathrm{Ca}^{2+}$ influx .... 41

Figure 3.11: Correlation among parameters of $\mathrm{Ca}^{2+}$ microdomain..... 43

Figure 3.12: Distribution of $\mathrm{Ca}^{2+}$ microdomain amplitude compared with published datasets.

Figure 3.13: Developmental changes in morphology of hair cell afferent synapses .............. 46

Figure 3.14: Ultrastructural changes of IHC ribbon synapses in development....................... 47

Figure 3.15: Capacitance response of IHC upon $\mathrm{Ca}^{2+}$ uncaging before and after onset of

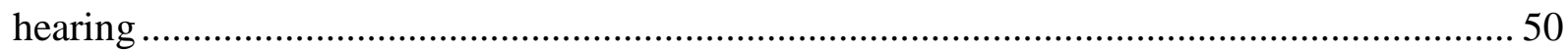

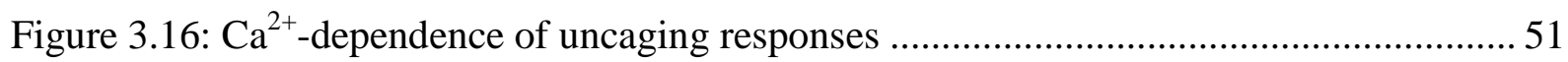


Figure 3.17: Re-implementation of a minimal kinetic model. .......................................... 52

Figure 3.18: Developmental change of apparent $\mathrm{Ca}^{2+}$ cooperativity in IHCs......................... 54

Figure 3.19: Spectrum of viral transduction in organ of Corti through embryonic injection .. 57 Figure 3.20: Expression of $\mathrm{vGpH}$ does not affect expression, and resembles the expression

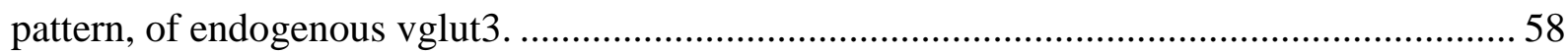

Figure 3.21: Bouton-like vglut1 immunoreactivity appears to be afferent terminals. ............. 59

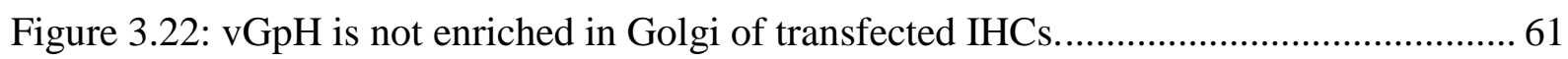

Figure 3.23: Calibrating $\mathrm{pH}$ of intracellular compartment by bath application of protonophore

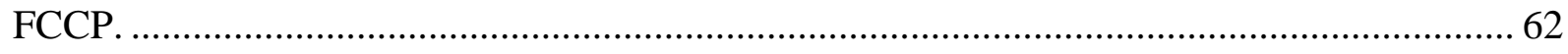

Figure 3.24: Depolarization of $\mathrm{vGpH}$ expressing IHCs reveals hotspots of vesicle fusion..... 64

Figure 3.25: Assessment of bleaching rate and signal-to-noise ratio of pHluorin imaging ..... 65

Figure 4.1: Properties of $\mathrm{Ca}^{2+}$ uncaging responses versus pre-flash $\left[\mathrm{Ca}^{2+}\right]_{\mathrm{i}} \ldots \ldots \ldots \ldots \ldots \ldots \ldots \ldots . . . . . . . . . . . .69$

Figure 4.2: How different $\mathrm{Ca}^{2+}$ influx manipulation affect release in nanodomain and

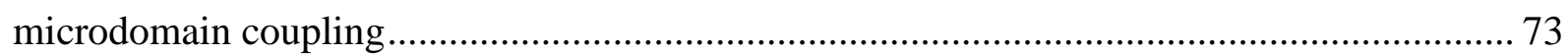

Figure 4.3: Proposed biological relevance in linearization of release cooperativity in IHC.... 75

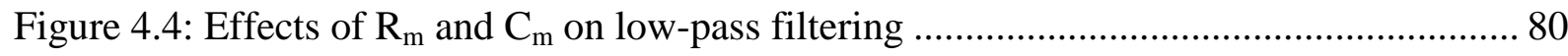

Figure 4.5: Comparison of RIBEYE-binding peptide intensity and baseline fluorescence of Fluo-5N 82 


\section{List of Abbreviations}

$\left[\mathrm{Ca}^{2+}\right]_{\text {post }}$

$\left[\mathrm{Ca}^{2+}\right]_{\text {pre }}$

a.c.

AMPA

AP

ATP

AZ

BAPTA

BDNF

cDNA

$\mathrm{C}_{\mathrm{m}}$

$\Delta \mathrm{C}_{\mathrm{m}}$

$\mathrm{CtBP} 2$

$\mathrm{CV}$

d.c.

DPTA

EGTA

EPSC

EPSP

$\mathrm{F}_{0}$

$\Delta \mathrm{F}$

$\Delta \mathrm{F} / \mathrm{F}_{0}$

$\mathrm{f}_{0}$

FCCP

FWHM

$\mathrm{g}_{\max }$

GSDB

GTP

HEPES

HSL

$\mathrm{I}_{\mathrm{Ca}}$

$\mathrm{i}_{\mathrm{Ca}}$

IHC

$\mathrm{K}_{\mathrm{d}}$

$\mathrm{k}_{\text {off }}$

$\mathrm{k}_{\mathrm{on}}$ post-flash $\left[\mathrm{Ca}^{2+}\right] / \mathrm{Ca}^{2+}$ concentration after flash photolysis

pre-flash $\left[\mathrm{Ca}^{2+}\right] / \mathrm{Ca}^{2+}$ concentration before flash photolysis

alternate current

$\alpha$-amino-3-hydroxy-5-methyl-4-isoxazolepropionic acid

action potential

adenosine triphosphate

active zone

1,2-bis(2-aminophenoxy)ethane-N,N,N',N'-tetraacetatic acid brain-derived neurotrophic factor

complementary deoxyribonucleic acid

membrane capacitance

change in membrane capacitance

C-terminal binding protein 2

coefficient of variation

direct current

1,3-diaminopropan-2-ol-tetraacetic acid

ethylene glycol-bis(2-amioethylether)-N,N,N',N'-tetraacetic acid

excitatory postsynaptic current

excitatory postsynaptic potential

baseline fluorescence

change in fluorescence intensity

normalized change in fluorescence intensity

cutoff frequency / corner frequency

Carbonyl cyanide 4-(trifluoromethoxy)phenylhydrazone

full-width at half maximum

maximum chord conductance

goat serum diluted buffer

guanosine triphosphate

4-(2-hydroxyethyl)-1-piperazineethanesulfonic acid

hue-saturation-luminescence

calcium current

unitary calcium current / single-channel calcium current

inner hair cell

dissociation constant

off rate / kinetic rate constant for $\mathrm{Ca}^{2+}$ unbinding

on rate / kinetic rate constant for $\mathrm{Ca}^{2+}$ binding 
MES

NA

NT-3

$\mathrm{OHC}$

PBS

PFA

$\mathrm{P}_{\mathrm{o}}$

PSF

$\mathrm{Q}_{\mathrm{Ca}}$

RGB

RIM

$\mathrm{R}_{\mathrm{m}}$

RNA

$\mathrm{R}_{\mathrm{p}}$

RRP

$\mathrm{R}_{\mathrm{s}}$

s.d.

s.e.m

SGN

SNARE

SNR

Syt1, Syt2, Syt 4

TEA

ttpr

$\mathrm{V}_{\mathrm{cmd}}$

vglut1, vglut3

vGpH

$\mathrm{V}_{\mathrm{m}}$
2-(N-morpholino)ethanesulfonic acid

numerical aperture

neurotrophin-3

outer hair cell

phosphate buffered saline

paraformaldehyde

opening probability (of channel)

point spread function

charge integral of calcium current

red-green-blue

Rab3-interacting molecule

membrane resistance

ribonucleic acid

parallel resistance

readily releasable pool

series resistance / access resistance

standard deviation

standard error of mean

spiral ganglion neuron

soluble N-ethylmaleimide-sensitive factor attachment protein receptors

signal-to-noise ratio

synaptotagmins I, II and IV

tetraethylammonium

time to peak release

command potential (voltage clamp)

vesicular glutamate transporters $1 \& 3$

vglut1-pHluorin fusion protein

membrane potential 


\section{Introduction}

\subsection{Sound and the mammalian ear}

Sound is an important means of communication in both human and other animals. Many animals also use the perception of sound as a tool in the evasion of predator, location of prey and even echolocation.

A sound is produced when a vibrating object periodically pushes against a medium, e.g air or water. This creates a series of compression and rarefaction, which propagates longitudinally in three-dimension. When one considers a sound wave at a particular location over time, it can be analyzed in terms of its frequency, amplitude, phase and waveform. Frequency and amplitude roughly correspond to the percept of pitch and loudness, while the waveform determines the "texture" or "quality" of the sound. Although sounds with pure sinusoidal waveform rarely occur in nature, a complex waveform can be mathematically represented as a combination of many sine waves of different frequencies and amplitudes through the process of Fourier transformation. Remarkably, a similar frequency analysis process occurs at the level of the inner ear, through a combination of passive and active mechanisms.

The frequency range of human sound perception ranges $20-20,000 \mathrm{~Hz}$. This range varies among mammalian species with a strong negative correlation to functional head size (Heffner and Heffner, 2008). High frequency hearing above $10 \mathrm{kHz}$ in mammals is a rather unique trait among vertebrates, with birds having an upper limit of $8-12 \mathrm{kHz}$ and reptiles, amphibians and most fish not hearing above $5 \mathrm{kHz}$ (Heffner and Heffner, 2008). The sound intensity the human auditory system can encode for ranges from 0 to $120 \mathrm{~dB}$ sound pressure level (SPL), an energy difference by 6 orders of magnitude. Moreover, it is able to extract a single sound source even when the signal to noise level is below $0 \mathrm{~dB}$, i.e. when the noise level is higher than the signal, which is commonly known as the "cocktail party effect". The precise mechanism by which our auditory system is able to achieve such remarkable feat is yet to be elucidated. We first begin from what is known about the mammalian ear. 


\subsubsection{Outer ear}

The mammalian outer ear consists of the pinna (auricula) and the ear canal (acoustic meatus) (Figure 3.1). The pinna focuses airborne sound into the opening of the ear canal, which conducts the sound wave towards the tympanic membrane located at the end of the canal. The pinna also produces spectral filtering effects to the sound wave, which was found to be important for sound source localization along the azimuth in humans (Batteau, 1967; LopezPoveda and Meddis, 1996).

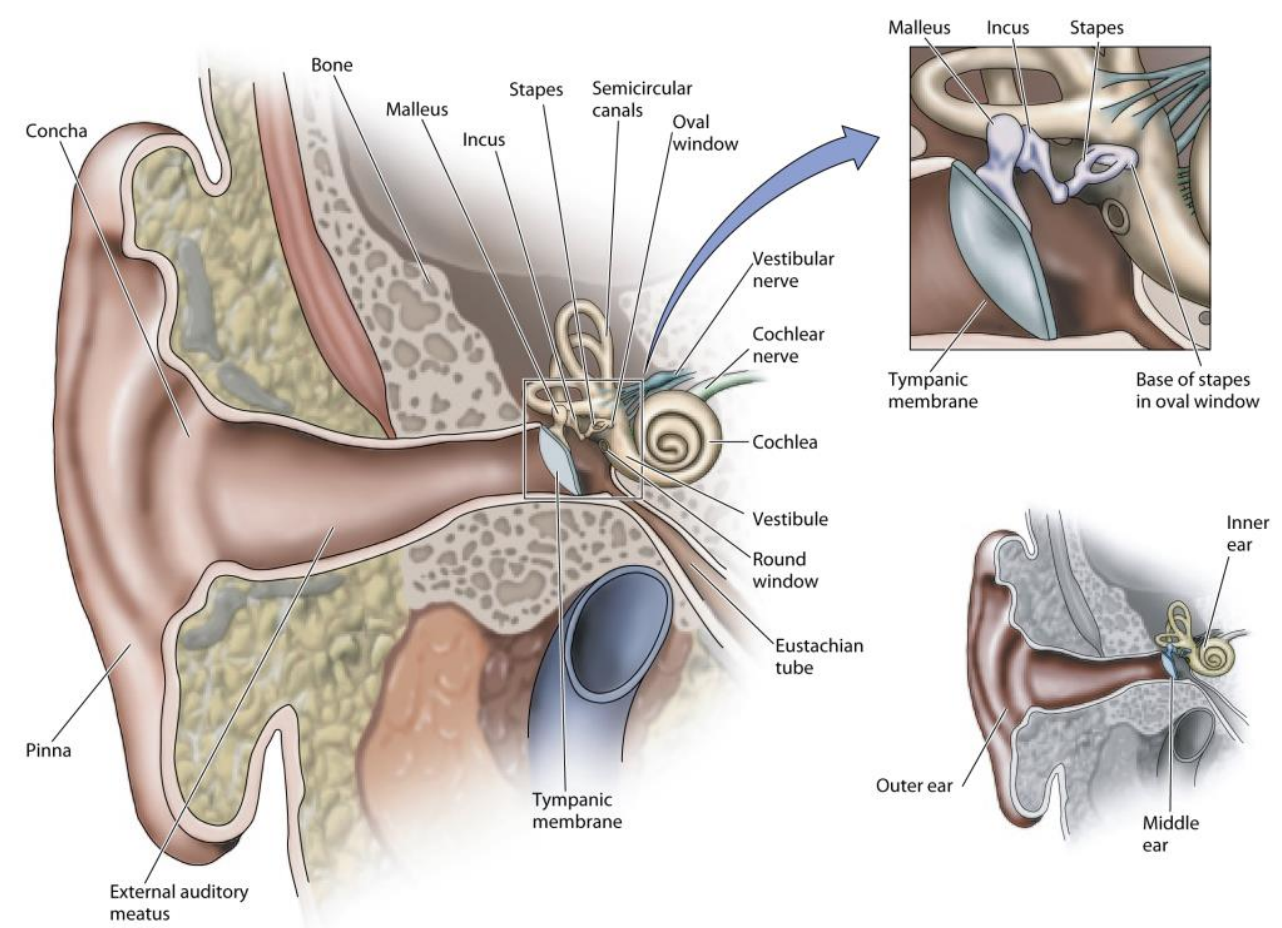

\section{Figure 1.1: Gross anatomy of a human ear.}

(illustration taken from Purves, 2004)

\subsubsection{Middle ear}

The tympanic membrane anatomically separates the outer and the middle ear, receives vibration collected by the outer ear, and transmits it to the cochlea through three ossicles in the middle ear - the malleus (hammer), incus (anvil), and stapes (stirrup). The main function of this arrangement is "impedance matching", because when sound wave travelling in medium with low mechanical impedance such as air to a high-impedance medium like water, most of the acoustic energy will be reflected. Through mechanical leverage of the ossicles and the 
difference in diameter between the tympanic membrane and the oval window, the middle ear increase the pressure by around 200-fold, ensuring the successful transmission of sound through the air-fluid boundary (Purves, 2004).

\subsubsection{Inner ear}

The inner ear, also called labyrinth due to its complexity in shape, consists of three major parts: vestibule, semicircular canals and cochlea (Gray, 1918), all of which are developed from the embryonic otic placode. The vestibule and the semicircular canals house the sensory organs of the vestibular system, which is responsible for the detection of gravity and motion.

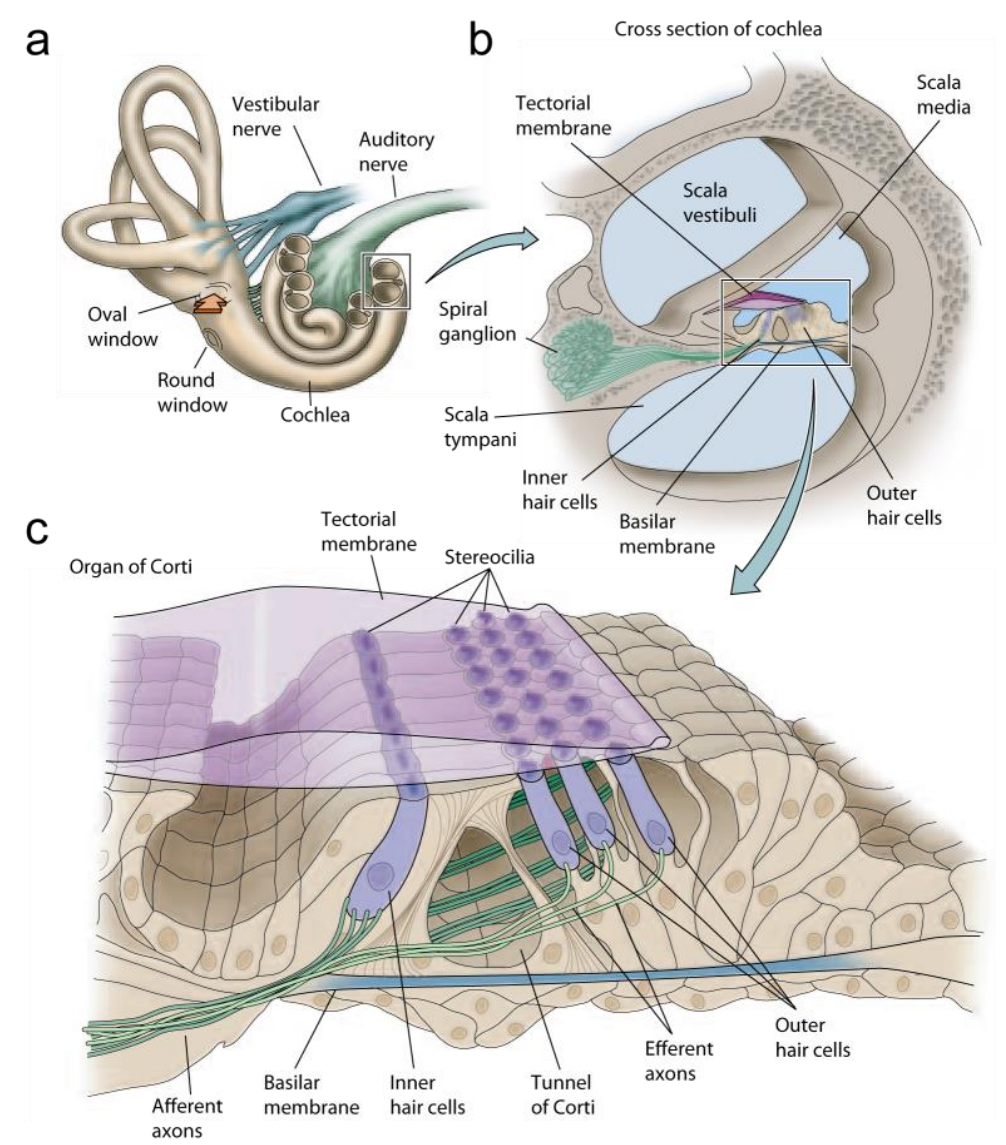

\section{Figure 1.2: Detailed anatomy of the cochlea and the organ of Corti.}

(illustration taken from Purves, 2004)

The snail-shaped cochlea, on the other hand, houses the sensory end organ of sound: the organ of Corti. Anatomically, the cochlea is a coiled structure with 2.5 turns in human. The number of turns varies with species, with mice having 1.75 turns and guinea pigs having 4 . In addition, 
the physical properties of the cochlea perform as a frequency analyzer, and greatly contribute to the tuning characteristics of auditory neurons.

The cochlea can be divided into three fluidic compartments: the scala vestibuli, scala media and scala tympani, are clearly seen in a transverse section of the cochlea (Figure 1.2b). The scala vestibuli and scala tympani are filled with a fluid called perilymph, and are connected at the apex of the cochlea at a structure called the helicotrema (illustrated in Figure 1.3). The scala media is, in contrast, filled with a potassium rich fluid called endolymph. At the border between the scala media and scala tympani is the organ of Corti. The organ of Corti lies between two acellular membranes the tectorial membrane and the basilar membrane (Figure $1.2 c)$.

When a sound wave arrives at the oval window, fluid in the scala vestibuli is vibrated and the pressure wave propagates along the length of the cochlea. A travelling wave is created on the basilar membrane, which is stiffer and narrower at the cochlear base but wider and more flexible towards the apex. Due to this arrangement, the travelling wave increases in amplitude and decreases in velocity along the cochlea, until a maximum point is reached (Figure 1.3). The position of maximal amplitude is related to the frequency of the stimulus, with higher frequency mapping to the base and lower frequency to the apex.

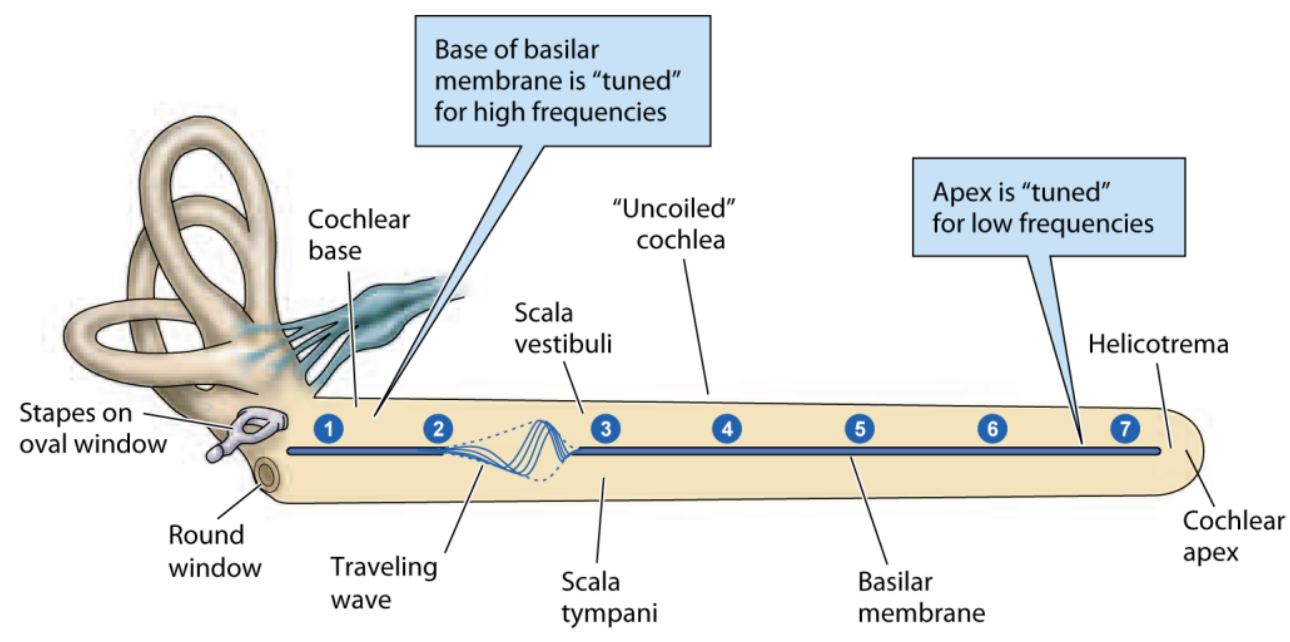

Figure 1.3: Frequency separation by the cochlea.

(illustration taken from Purves, 2004) 


\subsubsection{Sensory transduction at the organ of Corti}

The organ of Corti contains receptor hair cells. Along the length of the basilar membrane, there are one row of inner hair cells (IHCs) and three rows of outer hair cells (OHCs). The function of $\mathrm{OHCs}$ is the amplification of sound. The $\mathrm{OHC}$ specializes in its function through a negative stiffness in its hair bundle (Russell et al., 1992), and electromotility endowed by the protein prestin at its basolateral membrane (Zheng et al., 2000). This amplification process increases the sharpness of frequency tuning in individual auditory neurons. Only $5 \%$ of afferent innervation contacts the OHCs. In this regard, with more than $90 \%$ of the afferent innervation, the IHCs are the true sensory hair cell in hearing. Since different frequency components of sound are separated along the cochlea (also called tonotopy), each frequency is then detected by only one or a few adjacent IHC in the single row of IHC. IHCs are contacted by type I spiral ganglion neurons (SGNs). In the mature auditory system, each type I SGN only contacts a single active zone (AZ) of a single IHC, although each IHC is innervated by multiple SGNs.

On top of each sensory hair cell is the hair bundle, which consists of several rows of stereocilia arranged in a staircase fashion. Towards the top of the hair bundle, fibrous structures called tip-links connect stereocilia of adjacent rows. The vertical movement of the basilar membrane, through a pivotal action, is translated into a shearing motion between the tectorial membrane and the basilar membrane. This deflects the hair bundles of the sensory hair cells. Mechanoelectrical transduction channels mechanically coupled to the tip-links are opened and allow the influx of cations (mainly $\mathrm{K}^{+}$ion) into the hair cells which depolarizes the cell.

The depolarization of IHCs opens $\mathrm{Ca}_{\mathrm{V}} 1.3$ channels at the AZ of the basolateral plasma membrane, triggering exocytosis of glutamate-containing synaptic vesicles. Each $\mathrm{AZ}$ is innervated by a bouton-like terminal of the afferent SGN, containing a ring-like patch of $\alpha$ amino-3-hydroxy-5-methyl-4-isoxazolepropionic acid (AMPA) receptors (Meyer et al., 2009). The release of glutamate depolarizes the postsynaptic SGN and eventually elicits an action potential (AP), which is then transmitted to the auditory brainstem through the vestibulocochlear (VIII) nerve, conveying auditory information. Recently, it was 
demonstrated that most of the spontaneous excitatory post-synaptic potential (EPSP) in mouse SGNs can trigger an AP in vitro (Rutherford et al., 2012).

When studying the responses of SGN to sound stimuli in vivo by extracellular recording at the auditory nerves of anesthetized animals a great heterogeneity can be observed (Liberman, 1978; Sachs et al., 1989; Winter et al., 1990; Yates et al., 1990; Taberner and Liberman, 2005). In general, SGNs fire at a higher rate when a stimulus stronger than a certain threshold is presented. The frequency for which the neuron has the lowest threshold is called the characteristic frequency $(\mathrm{CF})$. For some neurons, the firing rate saturates at one to few tens of $\mathrm{dB}$ above the threshold at $\mathrm{CF}$, while the rate continues to increase in others (typically those with higher threshold). The spontaneous rate, defined as the firing rate in the absence of sound stimulus, also varies greatly among neurons (for mouse: 0 - 120 spikes/s, Taberner and Liberman, 2005), and was shown to be inversely related to the threshold of the neuron (Liberman, 1978; Winter et al., 1990; Taberner and Liberman, 2005). Interestingly, the dynamic range of individual SGN $(<43 \mathrm{~dB}$, Taberner and Liberman, 2005) is comparatively small to the range of sound intensity discernible by the organism $(\sim 120 \mathrm{~dB})$. The cohort of SGNs with different thresholds is believed to be responsible for encoding the full audible dynamic range of sound. Since SGNs with both high and low threshold exist at the same CF, a single IHC is thought to be innervated by SGNs of different response properties. This leads to the question about the origin of such heterogeneity, which will be discussed below (see section 1.3, p.9).

\subsubsection{The inner hair cell (IHC) ribbon synapse}

The synapses in IHCs have a specialized electron-dense structure called the synaptic ribbon, which is present also in other sensory cells performing high, sustained rates of neurotransmitter release, including photoreceptors and bipolar cells in the retina, mechanosensory hair cells in vestibular and auditory system and lateral line in fish, and electroreceptive cells (reviewed in Matthews and Fuchs, 2010). In addition, synaptic ribbons are also observed in the pineal gland (Hopsu and Arstila, 1965; Jastrow et al., 1997). The exact function of the ribbons remains elusive, but several non-exclusive hypotheses exists: (1) to organize and stabilize $\mathrm{Ca}^{2+}$ channels and vesicle release site at the active zone (Khimich et al., 2005; Hull et al., 2006; Frank et al., 2010; Sheets et al., 2011), (2) to support the rapid 
replenishment of readily releasable vesicles (von Gersdorff et al., 1996; LoGiudice et al., 2008; Frank et al., 2010; Snellman et al., 2011), (3) to prevent depletion of vesicle pool by restricting diffusion (Jackman et al., 2009), or (4) to promote synchronous multi-vesicular release or compound fusion of vesicles (Heidelberger et al., 1994; Fuchs, 2005; Matthews and Sterling, 2008; Graydon et al., 2011; Mehta et al., 2013).

More than $90 \%$ of the voltage-dependent $\mathrm{Ca}^{2+}$ influx in mouse IHCs are carried by $\mathrm{Ca} 1.3$ channels (Platzer et al., 2000; Brandt et al., 2003), which are observed to cluster at synaptic active zones (Brandt et al., 2005). $\mathrm{Ca}^{2+}$ influx through these channels are essential for triggering exocytosis. In addition, the IHC synapse contain active zone scaffolds, such as Bassoon and Piccolo (Khimich et al., 2005), and RIBEYE, the major constituent of the synaptic ribbon (Schmitz et al., 2000). Bassoon seems to perform additional function in anchoring the ribbon, as the loss of synapse-anchored ribbon was observed in photoreceptors and hair cells in a truncated Bassoon mutant (Dick et al., 2003; Khimich et al., 2005).

Other than sharing scaffold proteins such as Bassoon and Piccolo, the IHC ribbon synapse seems to bear little resemblance to neuronal synapses. For example, a recent study by Nouvian and colleagues (2011) has demonstrated that the IHC synapse seems to operate independently of the classical neuronal soluble N-ethylmaleimide-sensitive factor attachment protein receptors (SNAREs) SNAP-25, syntaxin-1, and synaptobrevin-1 or synaptobrevin-2. Moreover, while glutamatergic neurons mainly utilizes vesicular glutamate transporter isoforms vglut1 or vglut2, IHCs express vglut3 (Ruel et al., 2008; Seal et al., 2008), which is also found in glial cells and non-glutamatergic neurons (for a review on glutamate transporters, see Benarroch, 2010). Messenger RNA of synaptophysin, a synaptic vesicle marker, was not detected in the mature organ of Corti (Safieddine and Wenthold, 1999). The major $\mathrm{Ca}^{2+}$ sensors involved in synchronous transmitter release in neurons, synaptotagmins I and II (Syt1, Syt2), are apparently also missing in mature IHCs (Safieddine and Wenthold, 1999; Reisinger et al., 2011), although not without controversy (Beurg et al., 2010; Johnson et al., 2010). Putting all together, caution must be taken when relating synaptic transmission in neurons to the IHC ribbon synapse. 


\subsection{Postnatal development of mouse IHCs}

Mice are altricial animal and are born deaf until around the end of the second postnatal week (Mikaelian and Ruben, 1965; Mikaelian, 1979). During this period of postnatal development, IHCs undergo a dramatic switch in electric activity and synaptic properties. Prehearing IHCs fire $\mathrm{Ca}^{2+}$-mediated APs up to around postnatal day 11 (p11) (Kros et al., 1998), leading to robust glutamate release and drives bursting activity of the SGN (Tritsch et al., 2010) and downstream neurons in the auditory brainstem (Sonntag et al., 2009; Tritsch et al., 2010). Probably mediated through waves of ATP released by supporting cells (Tritsch et al., 2007), synchronous discharge of the SGN innervating IHCs in close proximity is thought to help the proper wiring and the preservation of tonotopy in the central auditory system. Similar patterned spontaneous activities were also observed in the developing retina, spinal cord, hippocampus and cerebellum, and are believed to be important in development of neural circuits (reviewed in Blankenship and Feller, 2010).

The density of voltage-dependent $\mathrm{Ca}^{2+}$ current in IHCs increases during the first postnatal week, and gradually decreases after p6 until around the onset of hearing ( p12) (Beutner and Moser, 2001; Brandt et al., 2003; Marcotti et al., 2003; Johnson et al., 2005). Early postnatal IHCs also express voltage-gated $\mathrm{Na}^{+}$conductance, which, together with the $\mathrm{Ca}^{2+}$ current, shapes and modulate the frequency of APs (Marcotti et al., 2003).

Small-conductance $\mathrm{Ca}^{2+}$-activated $\mathrm{K}^{+}(\mathrm{SK})$ channel SK2 is transiently expressed in the prehearing IHCs. The presence of SK channels is vital for the repolarization of $\mathrm{Ca}^{2+} \mathrm{AP}$ (Marcotti et al., 2004). Moreover, the SK channels are tightly coupled to the $\alpha 9 \alpha 10$ heteromeric nicotinic acetylcholine receptor (nAChR) and constitute part of the inhibitory efferent system originating from the medial superior olivery complex (Glowatzki and Fuchs, 2000; Brandt et al., 2003). During postnatal maturation, the efferent innervation of the IHCs is lost and instead the SGN afferents gain axo-dendritic efferent innervation from lateral olivery complex. 

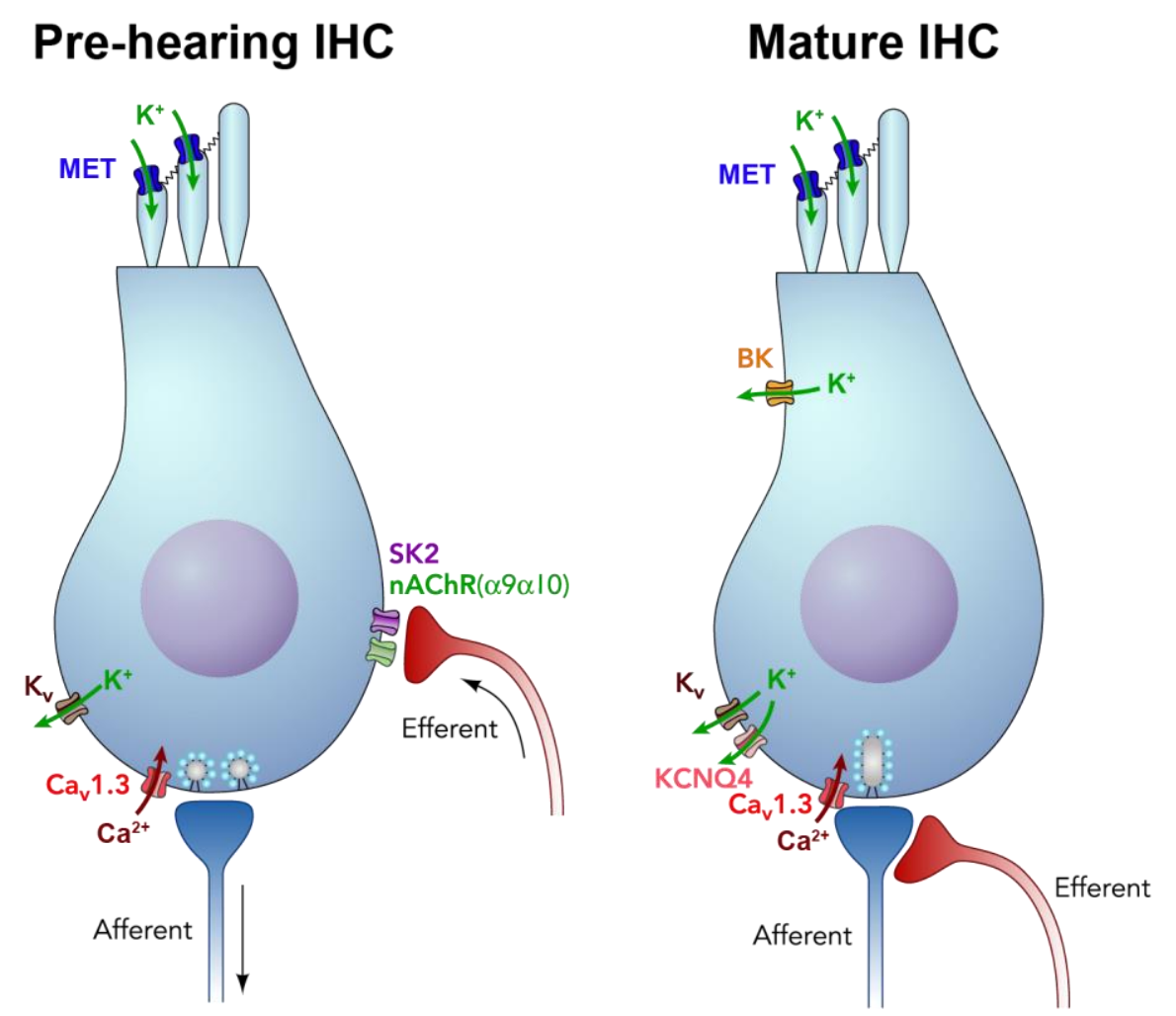

Figure 1.4: Schematic illustration of a pre-hearing and a mature IHC.

Illustration of major channels and receptors responsible for ionic conductances on the IHC, before and after the onset of hearing. (modified from Bulankina and Moser, 2012).

\subsection{Diversity of spiral ganglion neuron (SGN) response and possible mechanisms}

As mentioned above, the threshold and dynamic range of SGN in response to sound varies greatly, even among neurons showing similar $\mathrm{CF}$, a hint that they are innervating the same IHC or ones in close proximity. The spontaneous rate, measured as the firing rate in the absence of sound, showed negative relationship with response threshold in cat (Liberman, 1978), guinea pig (Winter et al., 1990), gerbil (Ohlemiller and Echteler, 1990), rat (El Barbary, 1991) and mouse (Taberner and Liberman, 2005). Liberman (1978) found that, in cat, SGN can be roughly divided into three groups based on their spontaneous rate (high, medium, low), each corresponding to a different sensitivity. Later, Liberman (1982) observed that the medium- and low-spontaneous rate (higher threshold) neurons preferentially innervated the neural (facing incoming afferent fibers, see Figure 1.2) side of IHCs, while high spontaneous 
rate neurons innervated the abneural (facing OHCs) side. This finding suggests the existence of a segregation of neural responses properties at the synaptic level. In the follow section, possible mechanisms will be discussed.
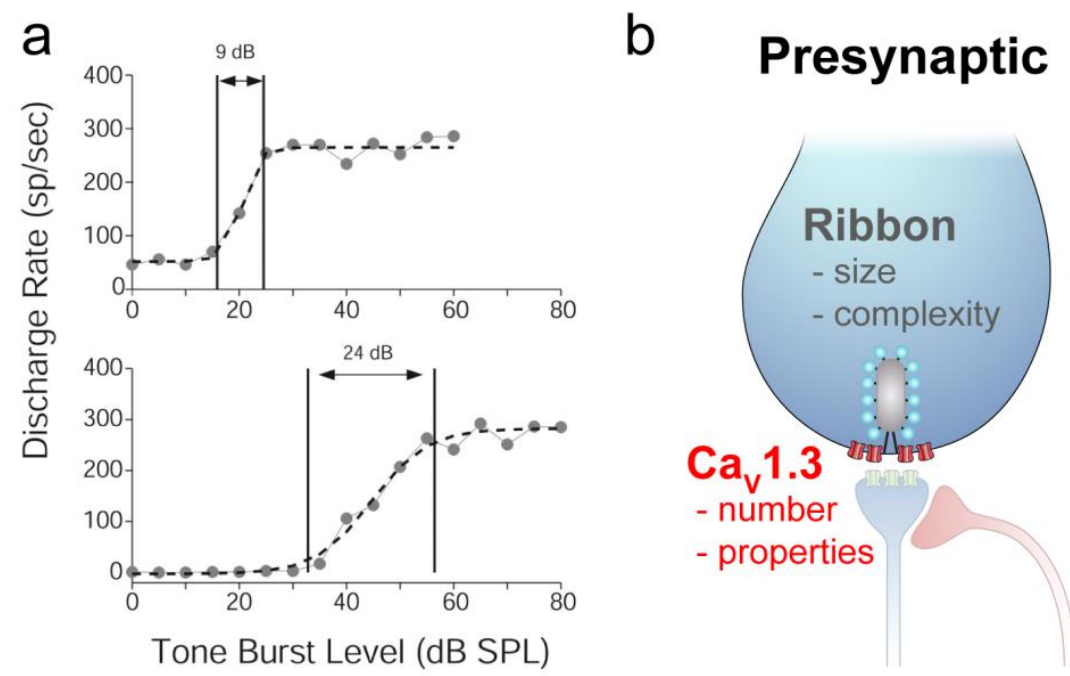

\section{Postsynaptic \& Efferent}

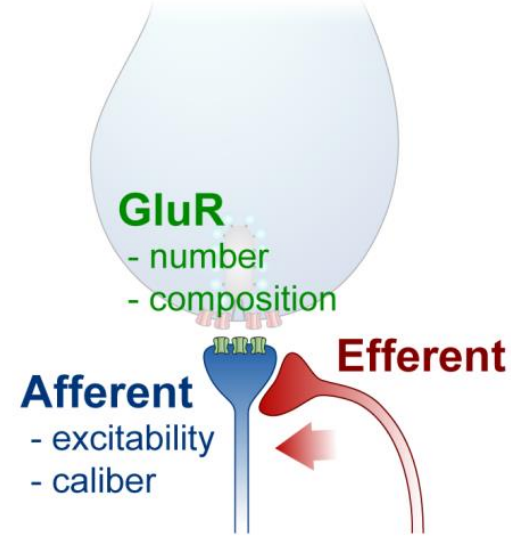

Figure 1.5: Diverse SGN response properties and examples of possible mechanisms

(a) Firing rate of two SGN in response to sound stimuli, demonstrating differences in spontaneous rate, threshold and dynamic range (marked by black lines) (Image taken from Taberner and Liberman, 2005). (b) Examples of possible mechanisms at the IHC-SGN synapse mediating the differences in SGN response properties. sp/sec: spike per second; GluR: glutamate receptor; $\mathrm{Ca}_{\mathrm{v}} 1.3$ : voltage-gated calcium channel of $\mathrm{Ca}_{\mathrm{v}} 1.3$ type

\subsubsection{Postsynaptic and efferent mechanisms}

The postsynaptic membrane of the IHC ribbon synapse contains mainly AMPA-type glutamate receptors (Glowatzki and Fuchs, 2002; Grant et al., 2010). Juxtaposed to each AZ in an IHC is a bouton-like terminal of the SGN that contains a single patch of glutamate receptor of a few hundred nanometers in diameter (Meyer et al., 2009). It is conceivable that variability in the number of AMPA receptor channels could lead to a heterogeneous size distribution in excitatory postsynaptic currents (EPSCs), and thus the ability of individual postsynaptic event in triggering an AP. A recent study (Liberman et al., 2011) indicated a spatial gradient of glutamate receptor cluster size using an antibody against AMPA-receptor subunits 2 and 3 (GluA2 \& GluA3; GluR2 \&GluR3 in earlier nomenclature, see Collingridge et al., 2009). Based on the non-rectifying and linear voltage dependence of EPSC amplitude (Glowatzki and Fuchs, 2002; Grant et al., 2010), it can be inferred that the AMPA receptors 
on SGN boutons contain GluA2 subunit (Cull-Candy et al., 2006). The expression of GluA3 and GluA4 in rat SGNs (Kuriyama et al., 1994; Knipper et al., 1996; Eybalin et al., 2004) and GluA4 in mouse (Huang et al., 2012) were also demonstrated.

In fact, postsynaptic recordings by Grant and colleagues (2010) have revealed that the average EPSC size in SGNs are highly variable in hearing (p19-21) rats (median amplitude from 97 to $473 \mathrm{pA}$ ), even when the presynaptic IHC were unstimulated. The authors attribute the heterogeneity in size distribution to the difference in number of vesicle released mediating each postsynaptic event (i.e. presynaptic mechanism, see below) while assuming a constant uniquantal EPSC size ( 50 pA). Nevertheless, difference in uniquantal EPSC size (and thus the size distribution) due to receptor composition and/or number could be a possible postsynaptic mechanism in regulating heterogeneous response properties of SGN.

On the other hand, the excitability of SGN is another possible mechanism leading to heterogeneous response properties. It was shown in the cat that low and medium spontaneous rate fibers have a smaller diameter than high spontaneous rate fibers (Liberman, 1982). The difference in surface to volume ratio would influence the length constant of electrotonic spread along the neurite, and thus affecting excitability. In vitro measurements from rat SGN afferent boutons, however, showed a small spike threshold $(-46 \pm 11 \mathrm{pA}, \mathrm{n}=8)$ and reliable spikes triggering $(>80 \%, \mathrm{n}=2)$ by spontaneous excitatory postsynaptic potentials (EPSPs; Rutherford et al., 2012).

Efferent activity, which would be disrupted in the in vitro preparation, can also modulate excitability of SGN afferents. Dopamine and its antagonists have been shown to modulate the spontaneous rate and threshold of SGN fibers in vivo, suggesting a tonic inhibition by dopaminergic LOC efferent system (Ruel et al., 2001). The whole LOC efferent function is probably more complex as the presence of other neurotransmitters, including acetylcholine, GABA and neuropeptides, have been implicated in the LOC neurons (Safieddine and Eybalin, 1992; Safieddine et al., 1996).

Both the difference in EPSC size and excitability will lead to a scaling of discharge rates (viz. change of success rate of events in triggering AP). This, together with saturation of evoked 
rates due to e.g. refractoriness of $\mathrm{AP}$, can produce a shift in spontaneous rate and dynamic range even if presynaptic release is assumed to be homogenous.

\subsubsection{Presynaptic heterogeneity}

Presynaptic mechanisms were also proposed to contribute to diverse response properties in SGNs. Labeling of single SGNs in cat (Merchan-Perez and Liberman, 1996) suggests a difference in ultrastructure of the $\mathrm{AZ}$ with respect to the spontaneous rate and threshold of the neuron. They found that high-spontaneous rate fibers tend to contact AZs with smaller ribbons, while low spontaneous rate fibers tend to contact larger ribbons. The degree of synchronous multivesicular release in hair cells, indicated by presence and proportion of multiphasic EPSCs, was also suggested to be a modulatory mechanism for the behavior of postsynaptic SGN (Grant et al., 2010).

Frank and colleagues (2009; Meyer et al., 2009) performed confocal $\mathrm{Ca}^{2+}$ imaging in IHCs, with which the $\mathrm{Ca}^{2+}$ signal mediated by voltage-dependent influx at spatially segregated AZs can be individually discerned. They found pronounced diversity in the amplitude and voltage dependence of these $\mathrm{AZ} \mathrm{Ca}{ }^{2+}$ signal. Even within a single $\mathrm{IHC}$, the amplitude of the $\mathrm{Ca}^{2+}$ signal could vary up to ten-fold. This suggests individual AZ within an IHC may differ in the number or voltage dependence of $\mathrm{Ca}^{2+}$ channels, which may underlie the heterogeneity in SGN response properties.

Unlike a neuron, which branches its axon and produce many bouton-like presynapses, the IHC is a small isopotential cell without apparent compartmentalization. How an IHC manages to differentially distribute $\mathrm{Ca}^{2+}$ channel and other synaptic components to individual AZs is a fascinating question. Therefore, it would be interesting to see how the properties of the synaptic $\mathrm{Ca}^{2+}$ signals develop in an IHC.

\subsection{Monitoring synaptic vesicle fusion in IHC}

The heterogeneity of the presynaptic $\mathrm{Ca}^{2+}$ signal is a promising candidate mechanism for explaining the diverse response characteristics of SGN to sound. The obvious next step would be to figure out whether or how heterogeneous presynaptic $\mathrm{Ca}^{2+}$ influx translates into differences in the actual release of neurotransmitter. However, traditional whole-cell capacitance measurements (e.g. Parsons et al., 1994; Moser and Beutner, 2000) cannot 
distinguish among fusion events at different AZs. More advanced electrophysiolgical methods such as on-cell capacitance measurements (Sheng et al., 2012) or recording of SGN afferent terminals (Glowatzki and Fuchs, 2002; Goutman and Glowatzki, 2007, 2011; Grant et al., 2010; Goutman, 2012) are technically challenging, therefore impractical for detecting AZ heterogeneity within a single IHC. Moreover, FM 1-43, a common optical probe for exocytosis, has been reported to pass through and block the mechanotransduction channels of hair cells (Nishikawa and Sasaki, 1996; Gale et al., 2001). The presence of FM dye in the cytosol means all membranous organelle will be labeled, hindering its use as a marker of recycling vesicles (but see Griesinger et al., 2002).
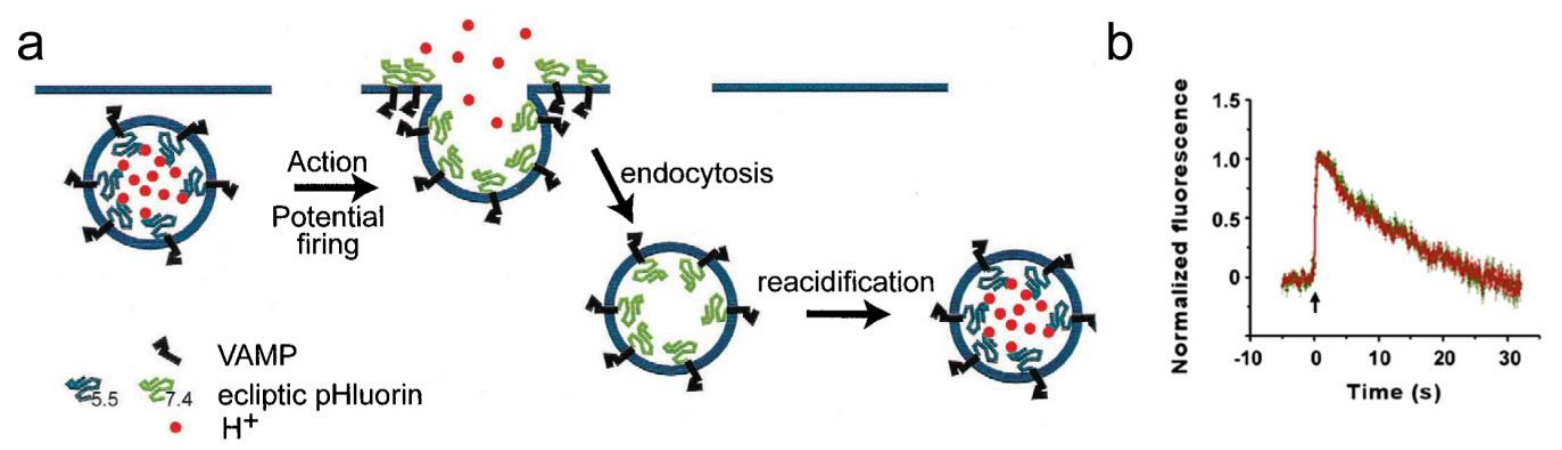

\section{Figure 1.6: Use of pHluorin to monitor vesicle recycling}

(a) Schematic showing how synaptopHluorin (a fusion between VAMP and ecliptic-pHluorin) changes its fluorescence intensity during vesicle cycling. Green and dark blue pHluoin symbols represent fluorescent and dark states of the protein. VAMP: vesicle associated membrane protein / synaptobrevin. (Image taken from Sankaranarayanan et al., 2000) (b) Fluorescence change of pHluorin upon single vesicle fusion. Arrow marks the time of stimulation. (Image taken from Zhu et al., 2009)

On the other hand, pHluorin, a pH-sensitive mutant of green fluorescence protein first developed by Miesenböck and colleagues (1998), has proven to be an useful tool in monitoring exocytosis and its recycling in cultured neuron (reviewed in Dreosti and Lagnado, 2011). The basis of this method is by targeting (super)ecliptic-pHluorin through fusion with a vesicular protein (Sankaranarayanan et al., 2000). The acidic environment in the synaptic vesicles ( pH5.6, Miesenböck et al., 1998) normally quenches the fluorescence of pHluorin (Figure 1.6a). Upon vesicle fusion, pHluorin molecules are exposed to the higher external $\mathrm{pH}$ of 7.4, causing an increase in fluorescence. The fluorescence signal then recovers during 
subsequent endocytosis and reacidification of vesicles. Using this method, single synapse (Fernandez-Alfonso and Ryan, 2008), and even single vesicle (Figure 1.6b, Zhu et al., 2009) resolution has been achieved. Expression of such a reporter in mammalian hair cell, however, has not been reported so far. The establishment of a pHluorin-based reporter in IHC will be valuable in probing exocytosis at single $\mathrm{AZ}$, paralleling the high resolution achieved in confocal $\mathrm{Ca}^{2+}$ imaging (Frank et al., 2009).

\subsection{Aim of the work}

The aim of the work includes: (1) further characterization of presynaptic $\mathrm{Ca}^{2+}$ signal in the mouse IHC ribbon synapse during postnatal development; (2) identify whether heterogeneity of the presynaptic $\mathrm{Ca}^{2+}$ signals arises during murine postnatal development; (3) characterization of the genetically-encoded reporter vglut1-pHluorin expressed in IHC through viral transduction, with the aim of monitoring vesicle fusion at single AZ of IHCs, and ultimately utilizing it in studying $\mathrm{Ca}^{2+}$-release coupling at single $\mathrm{AZ}$ through combination with $\mathrm{Ca}^{2+}$ imaging. 


\section{Material and Methods}

\subsection{Animals}

C57B1/6 mice (aged 6-30 days) mice were used for most experiments. $\mathrm{Ca}^{2+}$ channel $\alpha 1 \mathrm{Dnull}$ mutant mice (Dou et al., 2004) were used as negative control for immunohistochemistry. For vglut1-pHluorin experiments, embryonic transuterine otocyst injections were performed on CD1 dams mated with C57BL/6J males. All experiments complied with national animal care guidelines and were approved by the University of Göttingen Board for animal welfare and the animal welfare office of the state of Lower Saxony.

\subsection{Viral construct and virus production}

Cloning of vglut1-pHluorin expression vector was performed by Kirsten Reuter. Briefly, vglut1-pHluorin cDNA (kindly provided by Robert Edwards) were subcloned into AAVHBA-EWB vector using an EcoRI and a HindIII restriction site introduced through PCR. The AAV HBA- EWB vector (kindly provided by Sebastian Kügler) contains ITRs of AAV serotype 2 a human cytomegalovirus enhancer combined with a human $\beta$ actin promoter (HBA), an eGFP (enhanced GFP) reporter protein, a woodchuck posttranscriptional regulatory element (WPRE) and a bovine growth hormone (BGH) polyadenylation sequence.

Virus production was performed by Sebastian Kügler of the Viral Vectors Lab, Department of Neurology, University Medicine Goettingen as described previously (Kügler et al., 2007). Capsid proteins used were of serotype 1 and 2 (AAV1/2).

\subsection{Embryonic transuterine otocyst injections}

Embryonic transuterine otocyst injections were performed by Kirsten Reuter and Christiane Senger-Freitag. At postcoital day 11.5 pregnant dams were anesthetized with an intraperitoneal (i.p.) injection of $125 \mathrm{mg} / \mathrm{kg}$ body weight Ketamin (Ketamin Inresa, Inresa Arzneimittel $\mathrm{GmbH}$ ) and $2.5 \mathrm{mg} / \mathrm{kg}$ body weight Xylazin (Rompun®, Bayer), the anesthesia was maintained by applying $1 \mathrm{~L} / \mathrm{min}$ oxygen enriched with $0.4-1.2 \mathrm{Vol} \%$ Isofluran via a facial mask. Additionally, $50 \mathrm{mg} / \mathrm{kg}$ body weight carprofen were injected subcutaneously (s.c.). The anesthetized animal was shaved and the skin was sterilized at the abdomen and placed on a heated mat to prevent hypothermia. Once a stable level of anesthesia was reached, 
judged by the lack of paw withdrawal reflex, the laparoptomy was performed. The uterus was externalized and kept moist by applying warm isotonic solution. A cold light source was used to visualize the embryos inside the uterus, each embryo was gently positioned such that the left side points up, exposing the anterior cardinal vein and fourth ventricle. These two structures were used as landmarks for the otocyst injection. The virus solution, colored with 5 $\mathrm{mg} / \mathrm{ml}$ Fast Green (AppliChem), was then injected through a quartz glass injection pipette using a Pico Injector (Harvard Apparatus); The pipettes had been pulled on a P-2000 pipette puller (Sutter instruments) (settings: Heat 900, Fil 5, Vel 50, Del 126, Pul 175). The tips were snapped off at a diameter of $\sim 20 \mu \mathrm{m}$ with a rough angle of $45^{\circ}$ to create a thin sharp tip.

After the embryos were injected the uterus was re-internalized and the abdominal wall and skin were sutured with silk (size 06). The mice recovered and delivered 19-21 days postcoitum.

\subsection{Patch-clamp recordings}

IHCs from apical coils of freshly dissected organs of Corti were patch-clamped as described (Moser and Beutner, 2000). The standard pipette solution contained (in mM): 115 Csglutamate, 13 TEA-Cl, 20 CsOH-HEPES, $1 \mathrm{MgCl}_{2}, 2 \mathrm{MgATP}, 0.3 \mathrm{NaGTP}, 10$ EGTA, 0.4 Fluo-5N (or Fluo-4FF for fluorescence voltage-relationships (FV), action potentials (AP) and oscillating potentials; Penta-K ${ }^{+}$salts [Invitrogen] of dyes were used), and Carboxytetramethylrhodamine(TAMRA)-conjugated RIBEYE-binding peptide $(40 \mu \mathrm{M}$ monomer or $2 \mu \mathrm{M}$ tandem dimer Zenisek et al., 2004; Francis et al., 2011) for calcium imaging. In case of different EGTA concentration used, as stated in text or figure legends, Csglutamate concentration was adjusted for constant osmolarity. The extracellular solution contained (in mM): $104 \mathrm{NaCl}, 35 \mathrm{TEA}-\mathrm{Cl}, 2.8 \mathrm{KCl}, 5 \mathrm{CaCl}_{2}, 1 \mathrm{MgCl}_{2}, 10 \mathrm{NaOH}-\mathrm{HEPES}, 10$ D-glucose, $\mathrm{pH}$ 7.3. $\mathrm{CaCl}_{2}$ was $10 \mathrm{mM}$ for fluctuation analysis and $1.3 \mathrm{mM}$ for $\mathrm{Ca}^{2+}$ imaging of action potential and oscillating potential, with adjustment in $\mathrm{NaCl}$ concentration for osmolarity. For current clamp measurements, pipette solution contained (in mM): $145 \mathrm{~K}$ gluconate, $20 \mathrm{KOH}$-HEPES, $1 \mathrm{MgCl}_{2}, 2 \mathrm{MgATP}, 0.3 \mathrm{NaGTP}, 0.5$ EGTA, 0.4 Fluo-4FF (Penta- $\mathrm{K}^{+}$salts, Invitrogen), $2 \mu \mathrm{M}$ tandem dimer peptide (Francis et al., 2011); external solution (artificial perilymph) contained $144 \mathrm{NaCl}, 5.8 \mathrm{KCl}, 1.3 \mathrm{CaCl}_{2}, 1 \mathrm{MgCl}_{2}, 10 \mathrm{NaOH}-$ HEPES, 10 D-glucose, pH 7.3. 
Non-stationary fluctuation analysis (FA) was performed by Thomas Frank, similarly as previously described (Meyer et al., 2009) with bathed-applied BayK8644 (5 $\mu$ M, Tocris). Currents were low-pass filtered at $8.5 \mathrm{kHz}$ or $2.9 \mathrm{kHz}$ and sampled at $100 \mathrm{kHz}$ (FA, oscillating potentials) or $50 \mathrm{kHz}\left(\mathrm{Ca}^{2+}\right.$ currents), respectively.

An EPC-9 amplifier and "Patchmaster" software (HEKA Elektronik, Lambrecht, Germany) was used for most voltage-clamp measurements; and an EPC-10 amplifier was used for current-clamp measurements. For current-clamp measurements, stray capacitance compensation was first estimated by auto $\mathrm{C}_{\text {fast }}$ in voltage-clamp mode and then decreased by $3 \mathrm{pF}$ after switching into current clamp mode to avoid unwanted oscillation artifacts. For measurement of $\mathrm{Ca}^{2+}$ currents in voltage-clamp, additional $\mathrm{P} / \mathrm{n}$ protocol was performed to subtract leak current and residual uncompensated capacitance transients.

All voltages were corrected for liquid-junction potentials (LJP) and voltage-drops across series resistance $\left(R_{s}\right)$. LJPs were estimated using Patcher's Power Tools extension (Mendez and Würriehausen, Max-Planck-Institut für biophysikalische Chemie, Göttingen, Germany) for Igor Pro 6 (Wavemetrics, Lake Oswego, OR, USA). When potentials recorded in currentclamp were used as stimulus, the estimated LJP of the solutions used in current-clamp was first subtracted from the recorded traces to the true membrane potential. The estimated LJP for solutions used in voltage-clamp were added to obtain a "nominal" potential, which were then used as template traces in Patchmaster. When oscillating potentials were used as voltage clamp stimuli, true membrane potential after voltage drop across $\mathrm{R}_{\mathrm{s}}$ were estimated by numerically solving the following differential equation using the Euler method, implemented in Igor Pro 6:

$$
\frac{d V_{m}}{d t}=\frac{V(t)}{R_{s} C_{m}}-\frac{V_{m}}{R_{p} C_{m}}+\frac{V_{r e v}}{R_{m} C_{m}}
$$

where $\mathrm{V}_{\mathrm{m}}$ is the membrane potential, $\mathrm{V}_{\mathrm{rev}}$ is the reversal potential of leak conductance (fixed at $-80 \mathrm{mV}$ ), $\mathrm{V}(\mathrm{t})$ is the potential recorded by the amplifier (resampled to $1 \mu \mathrm{s}$ steps), $\mathrm{R}_{\mathrm{s}}$ and $\mathrm{C}_{\mathrm{m}}$ are the series resistance and membrane capacitance estimates from the $\mathrm{C}_{\text {slow }}$ compensation circuit, $R_{m}$ is the membrane resistance at holding potential (fixed at $3 G \Omega$ ), and $R_{p}$ is the parallel combination of $R_{s}$ and $R_{m}\left[1 /\left(1 / R_{s}+1 / R_{m}\right)\right]$. 
Current-voltage and fluorescence-voltage relationships (see below) were first transformed into an activation function, by factoring in the driving force for $\mathrm{Ca}^{2+}$ obtained from the linear portion $(-5$ to $+20 \mathrm{mV})$ of the $\mathrm{Ca}^{2+}$ current-voltage relationships:

$$
I_{C a}=g_{\max }\left(V-V_{\text {rev }}\right)
$$

where $V$ is the holding potential, $V_{r e v}$ is the reversal potential of $\mathrm{Ca}^{2+}$ and $g_{\text {max }}$ is the maximum chord conductance. The activation functions were subsequently fitted by a Boltzmann function:

$$
\frac{1}{1+\exp \left(\frac{V_{\text {half }}-V}{\text { slope }}\right)}
$$

where $V_{\text {half }}$ represents the half-activation potential and slope determines the steepness of the activation function.

Frequency dependence of $\mathrm{AC}$ oscillations $\left(\mathrm{V}_{\mathrm{m}}\right.$ or $\left.\mathrm{I}_{\mathrm{Ca}}\right)$ was fitted with a low-pass filter function:

$$
S=\frac{A}{1+f^{2} / f_{0}^{2}}
$$

where $S$ is the signal analyzed, $f$ and $f_{0}$ are the stimulus and cut-off frequencies and $A$ is a scaling constant.

Except for current-clamp experiments, cells with holding current greater than $-50 \mathrm{pA}$ were discarded.

\subsection{Confocal imaging of $\mathrm{Ca}^{2+}$ signal and pHluorin}

Confocal $\mathrm{Ca}^{2+}$ imaging was performed as described (Frank et al., 2009). In brief, synaptic $\mathrm{Ca}^{2+}$ microdomains were identified as hotspots of $\mathrm{Ca}^{2+}$ indicator fluorescence in $\mathrm{XY}$ scans using long (200 to $254 \mathrm{~ms}$ ) step depolarizations to $-7 \mathrm{mV}$. Closely-spaced AZs whose $\mathrm{Ca}^{2+}$ signals could not be unequivocally separated (i.e. $\mathrm{Ca}^{2+}$ microdomains with more than one nearby ribbon) were not considered for further analysis. We invoked $\mathrm{Ca}^{2+}$ influx by $20 \mathrm{~ms}$ step depolarizations to the potential eliciting the maximal $\mathrm{Ca}^{2+}$ channel activation (typically $-7 \mathrm{mV}$ ), which avoids differences in $\mathrm{Ca}^{2+}$ influx due to variability in voltage-dependence among IHC AZs. Line scans were performed at $0.7 \mathrm{kHz}$ with $10 \mathrm{mM}$ [EGTA] $]_{\mathrm{i}}$. Amplitudes of $\Delta \mathrm{F}_{\mathrm{F} 5 \mathrm{~N}}$ (in Figure 3.10) were estimated by the average of 3 peak-centered pixels in the last 
10 ms during depolarization. FWHM estimates were extracted from Gaussian fits on average $\Delta \mathrm{F}_{\mathrm{F} 5 \mathrm{~N}}$ profiles in the last $10 \mathrm{~ms}$ during depolarization. Voltage-dependence of synaptic $\mathrm{Ca}^{2+}$ microdomains was studied using the $\mathrm{Ca}^{2+}$ indicator Fluo-4FF for better sensitivity for lower levels of $\mathrm{Ca}^{2+}$ influx. The laser was positioned at the peak pixel of each $\mathrm{Ca}^{2+}$ microdomain as identified in XY scan (spot detection DiGregorio and Vergara, 1997; Frank et al., 2009)), and the cell was depolarized for $20 \mathrm{~ms}$ to different potentials with $5 \mathrm{mV}$ increments. The dependence of $\Delta \mathrm{F}$ amplitude on the membrane potential (FV relationship) was done by fitting the activation function with a Boltzmann function (see above). Spot detection was also used for $\mathrm{Ca}^{2+}$ signal under receptor potential like stimuli, and binned to an effective $7.5 \mathrm{kHz}$ (134 $\mu$ s bins) for higher temporal resolution. The binned traces were used for subsequent analysis with Fourier transformation.

Live imaging of organs of Corti transduced by the vglut1-pHluorin virus was performed with the same confocal setup with increased $488 \mathrm{~nm}$ laser power $(0.5-2 \%$ of maximum intensity) for the excitation of pHluorin, and increased detection gain for the photomultiplier tube (PMT, $750 \mathrm{~V}$ ). $\mathrm{NH}_{4} \mathrm{Cl}$ and $\mathrm{pH}$-calibration experiments were performed with solutions based on artificial perilymph (see above). For $\mathrm{NH}_{4} \mathrm{Cl}$ experiments, $50 \mathrm{mM} \mathrm{NH} \mathrm{Cl}_{4}$ replaces equal molar $\mathrm{NaCl}$ in the solution. For calibration of vesicular $\mathrm{pH}$, artificial perilymph was calibrated to $\mathrm{pH}$ 5.5, 6.5, 7.3, 8.0 and 9.0, with a $\mathrm{pH}$ meter. In non-neutral solutions, the $\mathrm{pH}$ buffer HEPES was replaced by MES ( $\mathrm{pH} 5.5 \& 6.5$ ) or tricine ( $\mathrm{pH} 8.0$ and 9.0). In addition, $20 \mu \mathrm{M}$ FCCP (carbonyl cyanide 4-(trifluoromethoxy)phenylhydrazone) solutions were diluted into the artificial perilymph from a $10 \mathrm{mg} / \mathrm{mL}$ acetone stock. FCCP solutions were applied through a bath perfusion system and $\mathrm{NH}_{4} \mathrm{Cl}$ solution was either bath perfused or applied through a large glass pipette. Patch-clamp capacitance measurements on vglut1-pHluorin expressing IHCs were performed using the standard Cs-glutamate intracellular solution containing $0.5 \mathrm{mM}$ EGTA, and extracellular solution containing $5 \mathrm{mM} \mathrm{CaCl}_{2}$.

\subsection{Flash photolysis of caged $\mathrm{Ca}^{2+}$}

Flash photolysis was performed by Tina Pangršič and the author as previously described (Nouvian et al., 2011). Briefly, to obtain step-wise increases in intracellular calcium, $100 \mu \mathrm{s}$ of pulsed laser light from a DPSL-355/1000 UV laser (Rapp OptoElectronic, Hamburg, Germany) were applied shortly after achieving the whole-cell configuration. Intracellular 
calcium concentration was measured by dual-wavelength ratiometric fluorimetry using the calcium indicator dye mag-fura-2 (Invitrogen, Darmstadt, Germany). The dye was excited with monochromator light alternating between 340 and $380 \mathrm{~nm}$ using a TILL Photonics system (Gräfelfing, Germany) and $\left[\mathrm{Ca}^{2+}\right]_{\mathrm{i}}$ was determined as previously described (Beutner et al., 2001). The pipette solution for flash-photolysis contained (in $\mathrm{mM}$ ): 83 Cs-gluconate, 16 TEA-Cl, 18 Cs-HEPES (pH 7.2), 0.3 mag-fura-2, 10 DM-nitrophen (gift of A. Leonov and C. Griesinger, Göttingen; or Calbiochem, Darmstadt, Germany), 5 DPTA (1,3-diaminopropan-2ol-tetraacetic acid) and $10 \mathrm{CaCl}_{2}$. The extracellular solution for flash-photolysis contained (in mM): $97 \mathrm{NaCl}, 35 \mathrm{TEA}-\mathrm{Cl}, 2.8 \mathrm{KCl}, 10 \mathrm{CaCl}_{2}, 1 \mathrm{MgCl}_{2}, 10 \mathrm{Na}-\mathrm{HEPES}, 1 \mathrm{CsCl}, 11.1$ D-glucose ( $\mathrm{pH}$ 7.2). The cells in which no $\mathrm{C}_{\mathrm{m}}$ responses could be elicited were excluded from analysis.

Capacitance responses were fitted with a single or double exponential function in the following form:

$$
\Delta C_{m}(t)=\left\{\begin{array}{cc}
\sum A_{i}\left(1-\exp \left(-k_{i}\left(t-t_{d}\right)\right)\right), & t>t_{d} \\
0, & t \leq t_{d}
\end{array}\right.
$$

for $i=1,2$ (double exponential) or $i=1$ (single exponential). Here, $\mathrm{A}_{\mathrm{i}}$ and $\mathrm{k}_{\mathrm{i}}$ are the amplitude and rate constants for the $i$ th component, respectively, and $t_{d}$ is the delay of the response after the flash. All responses were first fitted with a double exponential function. In cases where the rate constant of the two components differed by less than a factor of four, the responses were subsequently fitted by a single exponential.

\subsection{Immunohistochemistry}

Antibody staining and image acquisition for immunohistochemistry involving Cav1.3 was performed by Mark A. Rutherford. The freshly dissected apical cochlear turns were fixed for 1 hour in $4 \%$ formaldehyde diluted in phosphate buffered saline (PBS; contains $137 \mathrm{mM} \mathrm{NaCl}$, $2.7 \mathrm{mM} \mathrm{KCl}$ and $10 \mathrm{mM}$ phosphate buffer, $\mathrm{pH} 7.4$; Sigma) at room temperature, or, for staining involving $\mathrm{Ca}_{\mathrm{V}} 1.3,25$ minutes in $99 \%$ methanol at $-20^{\circ} \mathrm{C}$, and subsequently washed 3 times for 10 minutes each in PBS. Immunostaining was performed as described (Khimich et al., 2005). Explicitly, whole-mount preparations were incubated for $1 \mathrm{~h}$ in goat serum dilution buffer (GSDB; $16 \%$ normal goat serum, $450 \mathrm{mM} \mathrm{NaCl}, 0.3 \%$ Triton X-100, $20 \mathrm{mM}$ 
phosphate buffer, pH7.4) to block non-specific binding sites. Primary antibodies diluted in GSDB were applied overnight at $4^{\circ} \mathrm{C}$. Preparations were washed 3 times in a wash buffer (450 mM NaCl, 0.3\% Triton X-100, $20 \mathrm{mM}$ phosphate buffer, $\mathrm{pH} 7.4$ ) for 10 minutes each. Secondary AlexaFluor-labelled antibodies (Molecular Probes, 1:200) were applied for $2 \mathrm{~h}$ at room temperature. In cases of staining involving goat primary antibodies, goat serum in GSDB was replaced with a donkey serum and donkey secondary antibodies were used. After washing again three times for 10 minutes in wash buffer and once for 5 minutes in $5 \mathrm{mM}$ phosphate buffer, the preparations were mounted on a glass slide using Mowiöl 4-88 (prepared according to supplier's instruction; Carl Roth, Karlsruhe, Germany).

The following antibodies were used: mouse anti-CtBP2 (1:200, BD Biosciences), rabbit antiGluR2/3 (1:200, for GluA2/3, Chemicon), rabbit anti-Cav1.3 (1:150, Alomone Labs), goat anti-CtBP2 (1:150, Santa Cruz Biotech), mouse anti-GluR2 (1:75, for GluA2, Chemicon), mouse anti-Sap7f407 to Bassoon (1:1000, Abcam), rabbit anti-BSN1.6 to Bassoon (1:1000, E.D. Gundelfinger), mouse anti-Na ${ }^{+} / \mathrm{K}^{+}$ATPase alpha 3 subunit (1:200, Thermo Scientific, Catalog \#: MA3-915), guinea pig anti-vglut1(1:1000, Synaptic Systems, Catalog \#: 135304), mouse anti-synaptophysin (1:400, Synaptic Systems, Catalog \#: 101011), rabbit anti-vglut3 (1:500, Synaptic Systems, Catalog \#: 135203), rabbit anti-otoferlin (1:500, C terminal, Synaptic Systems), mouse anti-GM130 (1:100, BD Transduction, Catalog \#: 610822) and secondary antibodies conjugated with AlexaFluor fluorophores (1:200, Molecular Probes). Confocal images of immunolabeled vglut1-pHluorin transduced organs of Corti were acquired using a SP2 confocal microscope (Leica, Mannheim, Germany) with a 63x oil immersion objective. Confocal images of Cav1.3 immunolabeled IHCs were acquired using a SP5 confocal microscope (Leica, Mannheim, Germany) with $488 \mathrm{~nm}$ (Ar) and $594 \mathrm{~nm}$ (DPSS) lasers for excitation and a 100x oil immersion objective $(\mathrm{NA}=1.4)$.

Quantitative experiments were repeated until successful, i.e. if all antigens were stained with relative uniformity within each preparation. Preparations in which substantial bleaching in at least one of the fluorophore occurs were discarded. Each preparation yielded several images, each containing a row of 5-7 IHCs. Image stacks from two preparations per age, stained and acquired in parallel across age groups, were analyzed. Analysis of puncta intensity was performed in the optical section with the peak intensity and a Region-of-Interest (ROI) was 
determined by fitting a 2D Gaussian function on a $1 \mu \mathrm{m}^{2}$ region surrounding each RIBEYE/CtBP2 and Cav1.3 immunofluorescent spot as previously described (Frank et al., 2010). Comparison of plasma membrane versus ribbon-associated $\mathrm{Ca}_{\mathrm{V}} 1.3$ immunofluorescence was performed by first connecting all ribbons of an IHC which were within $3 \mu \mathrm{m}$ of each other in $3 \mathrm{D}$, and defining the connecting regions (each is a cylinder of $800 \mathrm{~nm}$ diameter) as basal plasma membrane. The average intensity of the plasma membrane region is then compared to that of the ribbon proximal region, defined as $3 \mathrm{D}$ spheres of $800 \mathrm{~nm}$ diameter centered at all connected ribbons. Three IHCs per age group were "reconstructed" and analyzed. Average voxel intensity of the whole 3D stack was used here as background and were subtracted from both averages before comparison.

Correlation maps (in Figure 3.20 and Figure 3.22) with extended color look up tables and their respective scatter plots were generated according to a concept described by Demandolx and Davoust (1997). In this extended dual-channel display, image was created in a hue-saturationluminance (HSL) colorspace concept rather than a linear addition of red, green and blue (RGB). First, the fluorescence intensities of the two channels ( $\mathrm{x}$ and $\mathrm{y}$ ) were independently normalized to an 8-bit range from 0 to 255 . The hue of each pixel was determined by mapping the value of $\arctan (y / x)$ from a range of $[0, \pi / 2]$ into magenta-red-yellow-green-cyan. The saturation was set to maximum and luminescence was calculated with a fuzzy "OR" logic as: $x+y-x y / 255$, which will also be in the range from 0 to 255 . The resulting HSL images were then converted to RGB for display using the ImageTransform operation in Igor Pro for display.

For deconvolution of confocal stacks (Figure 3.22), 10 iteration of the Richardson-Lucy algorithm was performed using the plugins DeconvolutionLab and PSF Generator (both from Biomedical Imaging Group, Lausanne Federal Institute of Technology) for ImageJ (Schneider et al., 2012). Point spread functions were generated using parameters of the SP2 microscope and a wavelength of $515 \mathrm{~nm}$ or $595 \mathrm{~nm}$, corresponding to the average wavelength in the detection window of either channel.

\subsection{Electron microscopy}

Electron microscopy was performed by Carolin Wichmann. Cochleae were explanted and perfusion-fixed for $1 \mathrm{~h}$ on ice with $4 \%$ PFA and $0.5 \%$ glutaraldehyde in 1x PBS, pH 7.2. 
Subsequently, the organs of Corti (apical cochlear coils) were explanted in 1x PBS and fixed overnight on ice with secondary fixative comprising $2 \%$ glutaraldehyde in $0.1 \mathrm{M}$ sodium cacodylate buffer, $\mathrm{pH}$ 7.2. The samples were washed in sodium cacodylate buffer and postfixed on ice for $1 \mathrm{~h}$ with $1 \%$ osmium tetroxide $((\mathrm{v} / \mathrm{v})$ in $0.1 \mathrm{M}$ sodium cacodylate buffer), followed by a $1 \mathrm{~h}$ washing step in sodium cacodylate buffer and three brief washing steps in distilled water. The samples were stained en bloc with $1 \%(\mathrm{v} / \mathrm{v})$ uranyl acetate in distilled water for $1 \mathrm{~h}$ on ice. After a brief wash with distilled water, samples were dehydrated at room temperature in increasing ethanol concentrations, infiltrated in Epon resin $(100 \% \mathrm{EtOH} / \mathrm{Epon}$ 1:1 (v/v), 30 and $90 \mathrm{~min} ; 100 \%$ Epon, overnight), and embedded for $48 \mathrm{~h}$ at $70^{\circ} \mathrm{C}$. Following conventional embedding $65-75 \mathrm{~nm}$ sections were obtained approaching from the anterior edge. Slices were postfixed and stained with uranyl acetate/lead citrate following standard protocols. Micrographs were taken with a JEOL electron microscope (JEM 1011) equipped with a Gatan Orius 1200A camera using the Digital Micrograph software package at an 8000-fold magnification.

Quantitative image analysis was performed as follows: For the size of ribbons, the longest axis of each ribbon in a section, excluding the membrane-bound rootlet region, was measured. For ribbon-associated synaptic vesicles the first row of vesicles around the ribbon with less than a vesicle diameter distance from the ribbon surface was counted. For the PSD length, pre- and postsynaptic membrane appositions decorated with PSD and a ribbon were measured. Three-dimensional reconstruction was performed on 4-6 serial $70 \mathrm{~nm}$ sections with the free software Reconstruct (Fiala, 2005).

\subsection{Computational Modeling}

RC circuit model for Figure 3.3 and kinetic model for flash photolysis data (Figure 3.17) were implemented in Igor Pro 6 by solving the respective sets of ordinary differential equations using a fifth-order Runge-Kutta method (IntegrateODE operation).

For a $n$-site sequential binding kinetic model (Beutner et al., 2001), a total of $n+2$ states were created (an array or "wave" with $\mathrm{n}+2$ points in one dimension), denoted here as $\mathrm{B}_{0}, \mathrm{~B}_{1}, \mathrm{~B}_{2,} \ldots$ $B_{n}$ and $B_{n+1}$ where the subscript indicates number of bound $\mathrm{Ca}^{2+}$ ions, except with $\mathrm{B}_{\mathrm{n}+1}$ being the released state. Rate of change for the species are: 


$$
\begin{aligned}
& \frac{d B_{0}}{d t}=-R_{0} \\
& \frac{d B_{i}}{d t}=R_{i}-R_{i+1}, \quad \text { for } i=0,1, \ldots, n \\
& \frac{d B_{\mathrm{n}+1}}{d t}=R_{n+1}
\end{aligned}
$$

where $R_{i}$ are rate of the reaction between species $B_{i}$ and $B_{i+1}$, given by:

$$
\begin{aligned}
& R_{i}=(n-i) k_{\mathrm{on}} a^{i}\left[\mathrm{Ca}^{2+}\right]\left[B_{i}\right]-(i+1) k_{\mathrm{off}} b^{i}\left[B_{i+1}\right], \text { for } i=0,1, \ldots, n \\
& R_{n+1}=\gamma\left[B_{n}\right]
\end{aligned}
$$

Here, $k_{o n}$ and $k_{\text {off }}$ are kinetic rate constants (in $\mu \mathrm{M}^{-1} \mathrm{~s}^{-1}$ and $\mathrm{s}^{-1}$, respectively) governing the forward and reverse binding reaction. These rate constants are modulated by the factors $a$ and $b$ depending on the number of bound $\mathrm{Ca}^{2+}$ ions to create the effect of cooperative binding. For most trials $a$ was set and fixed at 1 . The rate constant $\gamma$ denotes the rate of the final fusion step. Initial condition was set to $\{1,0,0, \ldots, 0\}$ as appropriate for low $\left[\mathrm{Ca}^{2+}\right]$ (assumed zero). The differential equations were then solved at different $\left[\mathrm{Ca}^{2+}\right]_{\mathrm{i}}$, and the time course of $\left[\mathrm{B}_{\mathrm{n}+1}\right]$ was fitted by the same function in section 2.6 to estimate release delay and rate constant. The time derivative of $\left[\mathrm{B}_{\mathrm{n}+1}\right]$ was used to estimate the time to peak release rate (ttpr).

A downhill simplex method was used to optimize the parameters as previously done (Beutner et al., 2001). A published algorithm written for C (Press et al., 1992 p.408-412) was adapted for Igor Pro 6. Conceptually, five (or six) initial sets of the four (or five) parameters $\left(k_{o n}, k_{o f f}\right.$, $b$ and $\gamma$, sometimes $a$ ) were first chosen. For each parameter set, the differential equations were evaluated at all $\left[\mathrm{Ca}^{2+}\right]$ from experiments, and the rate constant, delay and ttpr were compared with experimental data. The deviation was calculated as an error function combining the root-mean-square difference in all three estimates (in either normal or log scale). Error minimization occurs as an "amoeboid" motion in parameter space. A new set of parameters were picked by "reflecting" the parameter set with the greatest error in parameter space. If the error with the new set is lower than the worst old set, then the new set is adopted. If the error with the new set is lower than even the best old set, a further "elongation" along the direction is performed. If the new set is worse than the worst old set, a contraction around 
the best old set is performed. This process continues until the error of all parameter sets are within a defined fractional tolerance (set to $10^{-4}$ in this implementation).

\subsection{Data analysis and statistical tests}

Data are presented as mean \pm s.e.m., unless otherwise specified. Igor Pro 6 (Wavemetrics, Lake Oswego, OR, USA) was used for analysis of functional imaging and immunohistochemical data. Single unit recordings and ratiometric $\left[\mathrm{Ca}^{2+}\right]$ measurements (in flash photolysis) were analyzed using MatLab (The MathWorks, Natick, MA, USA). Electron microscopy data was analyzed in Microsoft Excel (Microsoft, Redmond, WA, USA). Figures were assembled for display in Adobe Photoshop and Illustrator softwares. Normality of data set was assessed with the Jarque-Bera test. F test was used to assess equality of variance in normally distributed data set. Unpaired, two-tailed Wilcoxon rank test (Mann-Whitney test) was used to compare data significantly different from a normal distribution, or when variances of experimental groups were unequal. In case of normally-distributed equal-variance data, student's unpaired, two-tailed t-test was used to compare two samples ( $*$ indicates $\mathrm{p}<0.05$ ). Comparison of dispersion was performed with a modified Levene's test (Brown and Forsythe, 1974), using median instead of mean for improved robustness under non-normality. In cases where sample means were unequal or for comparison across sets of experiments, relative dispersion was compared either by first normalizing to the median of each dataset or by testing on log-transformed data (Donnelly and Kramer, 1999). One way ANOVA followed by Tukey's test (for non-stationary fluctuation analysis) was used to detect differences in multiple comparisons. 


\section{Results}

\section{1 $\mathrm{Ca}^{2+}$ Signal in hair cell during development}

\subsubsection{Naturalistic electrical activities and underlying calcium signals}

As described previously (Kros et al., 1998; Beutner and Moser, 2001; Johnson et al., 2005), slow $\mathrm{Ca}^{2+}$ action potentials were observed in IHCs of prehearing mice under current clamp condition (Figure 3.1A, upper and middle panels). With the help of a fluorescent RIBEYEbinding peptide (Francis et al., 2011), one can position the confocal laser beam at the presynaptic ribbon-type $\mathrm{AZ}$ and visualize the synaptic $\mathrm{Ca}^{2+}$ transient during such action potentials (Figure 3.1A lower panel). The transients were short-lived and temporally coincided with the action potentials.

I also compared the $\mathrm{Ca}^{2+}$ transient evoked by an action potential template and a more standard step depolarization protocol (Figure 3.1B). In these experiments, the IHCs were voltageclamped while pharmacologically inhibiting of voltage-dependent $\mathrm{K}^{+}$and $\mathrm{Na}^{+}$conductances. The cells were then presented with a $20 \mathrm{~ms}$ step depolarization to $-7 \mathrm{mV}$ and a previously recorded $\mathrm{Ca}^{2+}$ action potential in an alternate manner. Figure 3.1B shows the whole cell $\mathrm{Ca}^{2+}$ current and the $\mathrm{Ca}^{2+}$ signal at 8 active zones in 4 IHCs. Individual fluorescence traces were averages of 10 repetitions and the grand average of the 8 traces were shown as a black line ( \pm s.e.m. as shaded area). As shown in the lower panel, the amplitude of synaptic $\mathrm{Ca}^{2+}$ signal during an AP is comparable to that of the step depolarization, a protocol that mobilizes the readily releasable pool within milliseconds (Moser and Beutner, 2000). 
a

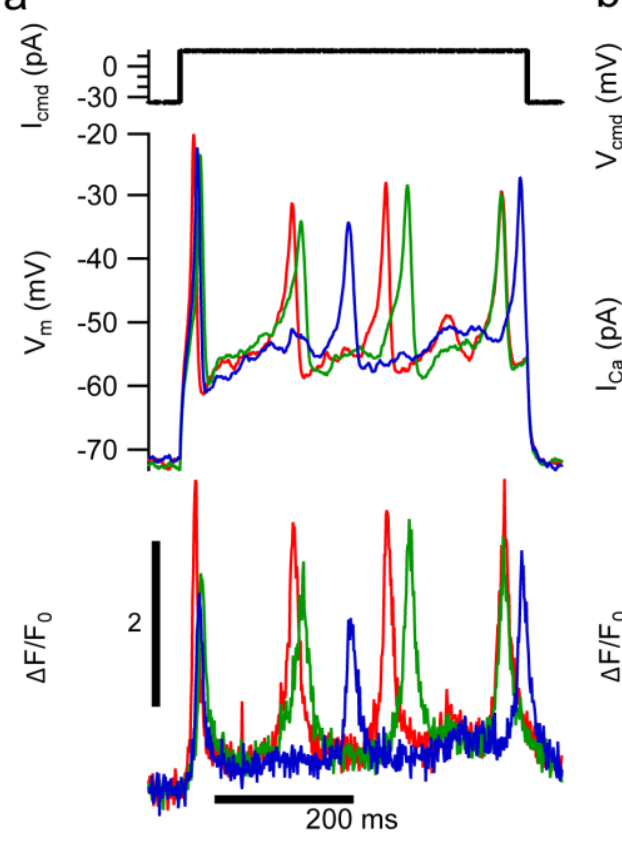

b
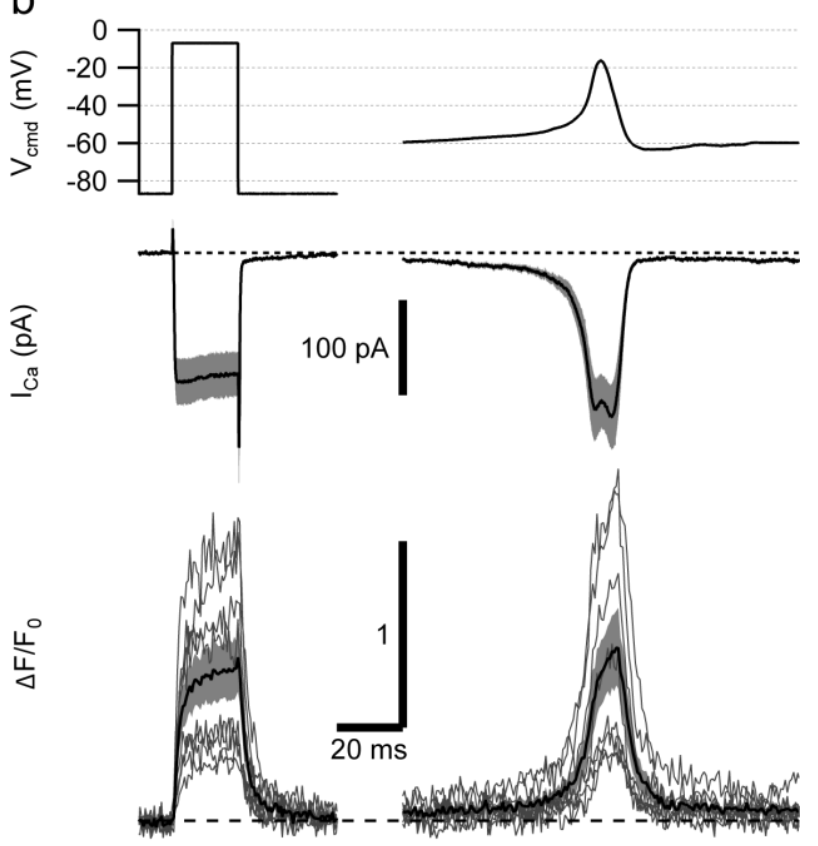

Figure 3.1: $\mathrm{Ca}^{2+}$ signal in IHC of prehearing animal.

(a) Injection of small depolarizing current (upper panel) to a pre-hearing IHC (p10) elicited action potentials (middle panel). Lower panel shows the fluorescence change of Fluo-4FF at a synaptic ribbon, where a $\mathrm{Ca}^{2+}$ transient (lower panel) was observed during each action potential (500 $\mu \mathrm{M}$ [EGTA]i). (b) $\mathrm{Ca}^{2+}$ current and $\mathrm{AZ} \mathrm{Ca}{ }^{2+}$ signal of pre-hearing IHCs (p9-10) evoked by a step depolarization (left) and a recorded $\mathrm{Ca}^{2+}$ action potential (right). Average $\mathrm{Ca}^{2+}$ current (middle panel; \pm s.e.m.; $\mathrm{n}=8$ ) was recorded in the presence of TEA, $\mathrm{Cs}^{+}$, linopirdine, apamine and TTX, and further isolated by a P/N protocol. Bottom panels show individual and average traces of simultaneously measured $\mathrm{Ca}^{2+}$ signal (change in Fluo-4FF fluorescence). Each trace is an average of 10 repetitions.

While the $\mathrm{Ca}^{2+}$ action potential is the natural electric activity of a pre-hearing IHC, the natural environment for a mature IHC would be a sound evoked receptor potential. In order to study how current through mechanotransduction channel of hair bundle affects membrane potential, I created rectifying sinusoidal waveforms reminiscent of recorded transduction current (Jia et al., 2007; Johnson et al., 2011). These waveforms (Figure 3.2) have varying amplitudes and frequency, and were used as templates for whole-cell current-clamp measurement of IHCs in vitro. Since there are different estimates of resting membrane potential in IHC (Dallos, 1985; Oliver et al., 2003; Johnson et al., 2011), three holding potentials (-77mV, $-67 \mathrm{mV},-57 \mathrm{mV}$ ) were used in these experiments. Before each stimulus, the IHC was voltage-clamped to one of the three holding potentials, and then switched to current-clamp mode using the "gentle 
switch" function of EPC10 amplifier, which initiates with the holding current at the potential during voltage-clamp mode. The stimulus template was then applied "on top of" the holding current.
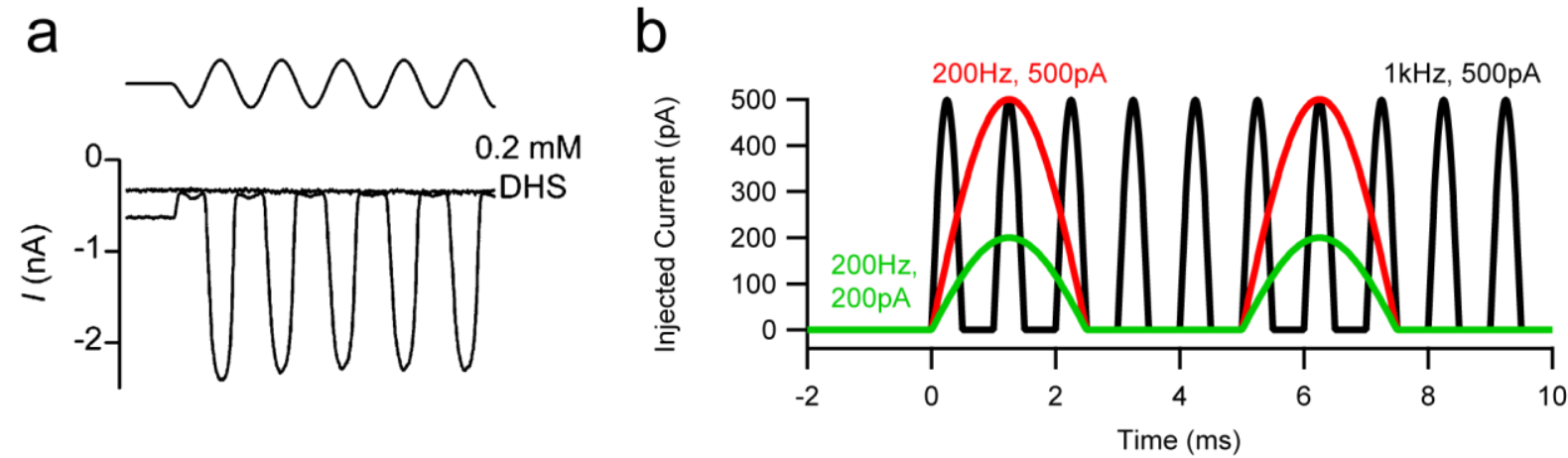

Figure 3.2: Current template resembling transduction current.

(a) Mechanotransduction current (lower panel) measured by Johnson and colleagues (2011) in an IHC during a $200 \mathrm{~Hz}$ fluid jet stimulation (upper panel). Perfusion of $0.2 \mathrm{mM}$ dihydrostreptomycin (DHS), a blocker of mechanotransduction channel, abolishes all transduction current (flat trace in lower panel). (b) Examples of current templates with varying amplitude and frequencies.

Figure 3.3a shows the responses of two p15 IHCs to injection of mechanotransduction-like currents. For clarity, only responses with one holding potential $(-77 \mathrm{mV})$ are shown on Figure 3.3a. Firstly, with increasing stimulus frequency, the amplitude of the oscillation (a.c. component) decreases (Figure 3.3a). The a.c. component of the response was quantified by measuring the peak-to-peak difference of the response. Secondly, both the a.c. component and the mean depolarization (d.c. component) increased with amplitude of the injected current. The d.c. component was measured as the difference between mean steady-state potential and the holding potential. 

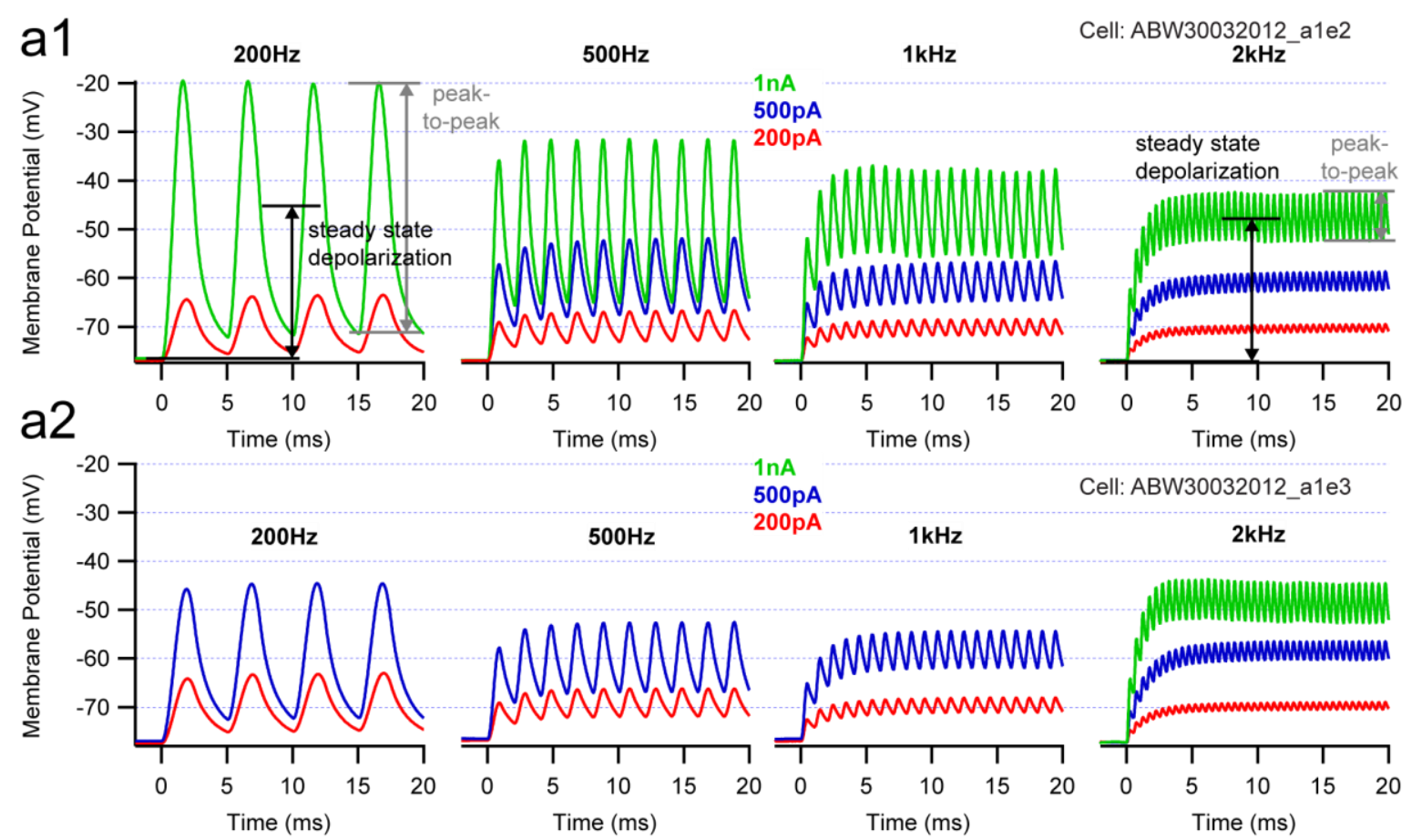

Cell: ABW30032012_a1e3

200pA $\quad 1 \mathrm{kHz}$

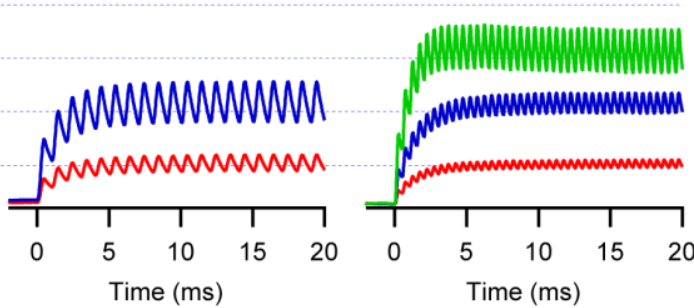

b

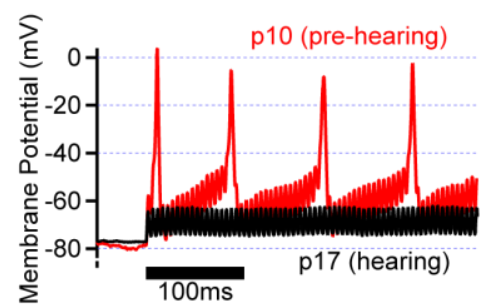

C

d
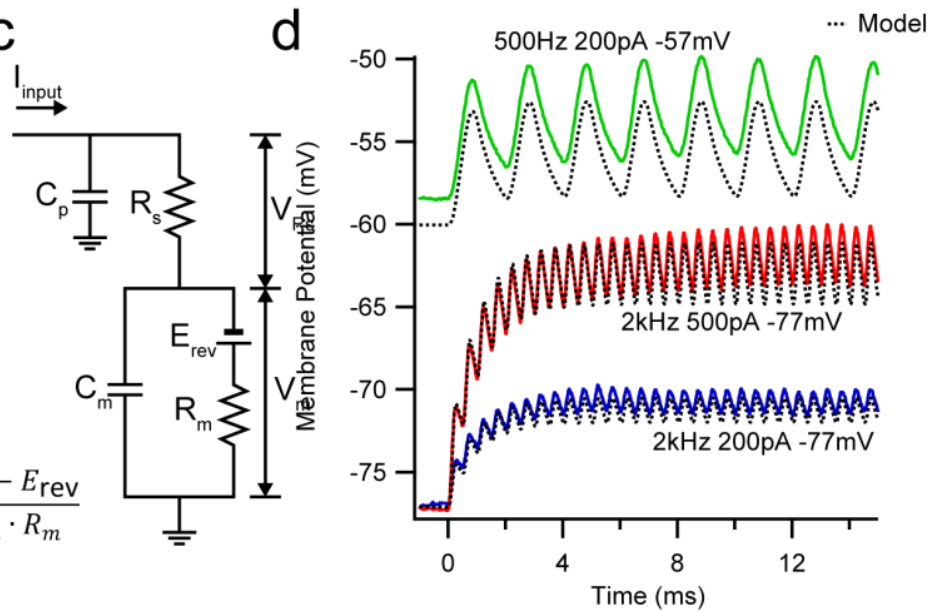

Figure 3.3: Response of IHCs to current injections mimicking natural mechanoelectrical transduction.

(a1) and (a2) averaged voltage response of two representative p15 IHCs to naturalistic (rectified sinusoidal) current injection of different amplitudes and frequencies at a holding potential of $-77 \mathrm{mV}$. D.c. component of the response was measured as the mean depolarization at steady-state and the a.c. component as the peak-to-peak potential difference. Both d.c. and a.c. component increased with amplitude of injected current but the a.c. component decreased with frequency. (b) Different responses of a pre-hearing (p10, red) and a hearing (p17, black) IHC to the same stimuli (200 Hz, $200 \mathrm{pA})$. (c) RC circuit model of the whole-cell patch-clamp configuration. (d) Estimation of voltage response in silico by solving the two ordinary differential equations using passive electric properties of the IHC at holding potential. 
Pre-hearing IHC responded with much stronger depolarization and showed action potentials with the smallest (200 pA) injection amplitude (Figure 3.3b).

Interestingly, most of the responses could be well reproduced in silico with a simple resistance-capacitance (RC) circuit model of the whole cell patch-clamp configuration (Figure 3.3

c-d), only using the passive electric properties $\left(C_{m}, E_{r e v}, R_{m}\right)$ of the IHC, those of the recording electrode $\left(\mathrm{R}_{\mathrm{s}}, \mathrm{C}_{\mathrm{p}}\right)$ and the current template $\left(\mathrm{I}_{\text {input }}\right)$ as input parameters. The model is described by the two differential equations shown in Figure 3.3. $R_{s}$ and $C_{m}$ were estimated by the CSlow compensation in Patchmaster software. $E_{\text {rev }}$ and $R_{m}$ were estimated from a $10 \mathrm{mV}$ biphasic test pulse around the holding potential during the brief voltage-clamp period before each stimulus. $\mathrm{C}_{\mathrm{p}}$ was taken as the $3 \mathrm{pF}$ of uncompensated stray capacitance during current clamp.

The measured a.c. and d.c. components of the responses from the two IHCs in Figure 3.3 are plotted in Figure 3.4, emphasizing the frequency and amplitude dependence of the a.c. component. Considering the IHC membrane acts as a low-pass filter, I fitted the a.c. response to each family of stimuli (same amplitude and holding potential) by a filter function in the form $\mathrm{V}=\mathrm{V}_{0} /\left(1+\mathrm{f}^{2} / \mathrm{f}_{0}{ }^{2}\right)^{1 / 2}$, where $V$ is the a.c. response amplitude, $f$ and $f_{0}$ are the stimulus and cutoff frequencies in $\mathrm{Hz}$, and $V_{0}$ is $V$ at $f=0$. At higher frequencies, the a.c. component decreased at the typical rate of $-20 \mathrm{~dB} / \mathrm{decade}$, where the absolute amplitude was independent of holding potential. At lower frequencies, the amplitude decreased with higher holding potential, which increased the cutoff frequency $f_{0}$ from fit. This is probably due to a decrease in the membrane time constant upon $\mathrm{K}^{+}$channel activation (Kros et al., 1998). Table 1 summarizes the $f_{0}$ of responses from all mature (p15-17) IHCs measured. D.c. components increased with stronger injection amplitude, but decreased with more positive holding potentials (Figure 3.4). Again, a more depolarized potential led to $\mathrm{K}^{+}$channel activation, and shunted the injected depolarizing current. 

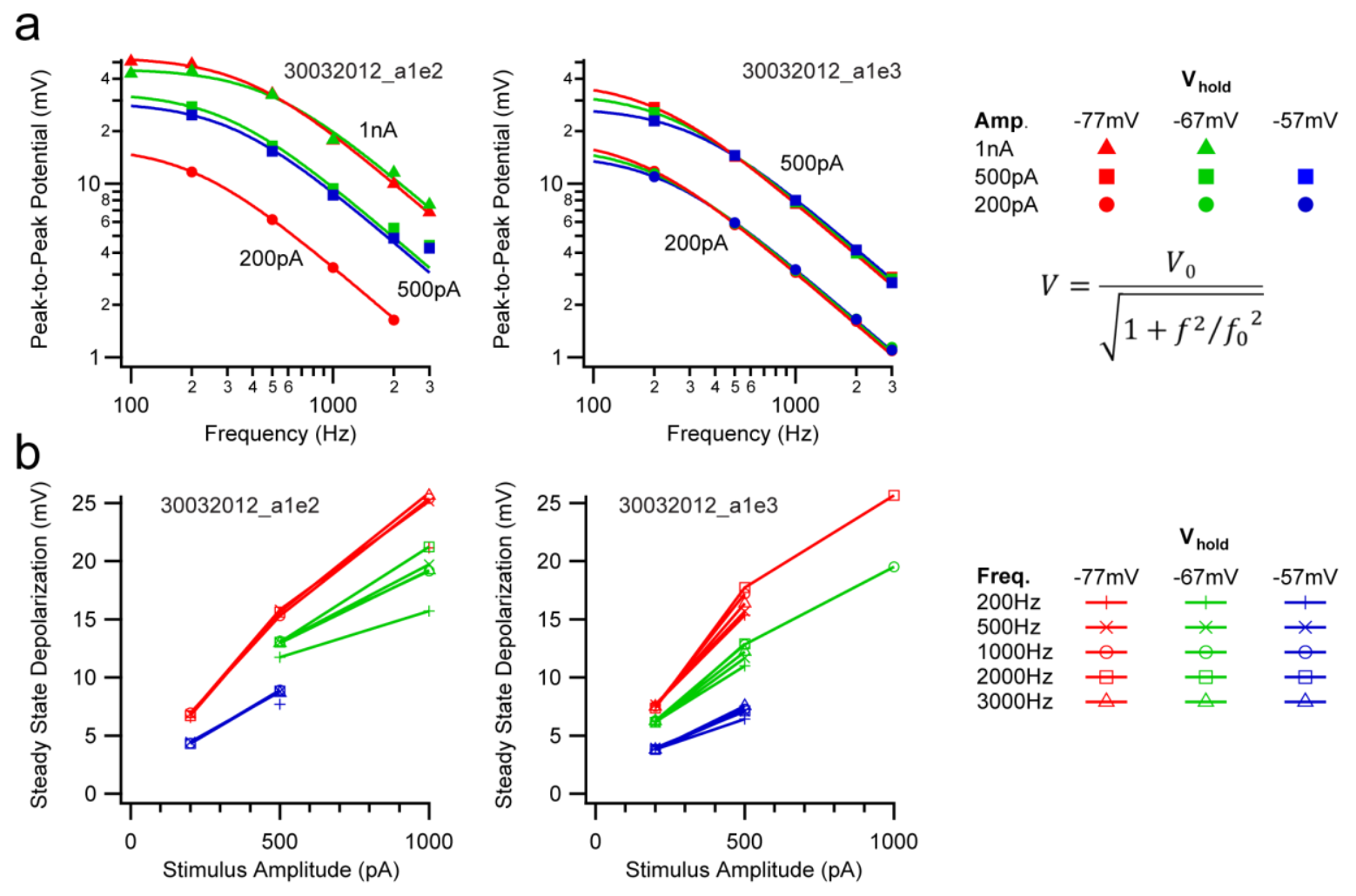

Figure 3.4: Peak-to-peak and steady-state depolarization of IHC voltage responses

(a) Log-log plot of the peak-to-peak oscillation (a.c. component) in membrane potential against stimulus frequency of the same IHCs in Figure 3.3. Solid lines represent best fits of a low pass filter function $\mathrm{V}=$ $\mathrm{V}_{0} /\left(1+\mathrm{f}^{2} / \mathrm{f}_{0}^{2}\right)^{1 / 2}$ to data points acquired under the same stimulus amplitude and holding potential. Only datasets with at least 4 data points were plotted here. (b) Normal-normal plot of steady-state depolarization (d.c. component) against stimulus amplitude. The d.c. component increases with stimulus amplitude but decreases with more positive holding potential.

I then looked at the $\mathrm{IHC} \mathrm{Ca}^{2+}$ current and the presynaptic $\mathrm{Ca}^{2+}$ signal $\left(\mathrm{Ca}^{2+}\right.$ microdomain $)$ under such oscillating potentials. In an attempt to separate their frequency-dependence from filtering contributed by the RC circuitry of the IHC membrane (see above), command voltage templates of various frequencies but identical amplitude were generated. To do so, the response of a p15 IHC to a $200 \mathrm{~Hz}, 1 \mathrm{nA}$ rectified-sinusoidal current was "compressed" along the time axis to create stimulus of higher frequencies. These stimuli were then applied in voltage-clamp while monitoring the $\mathrm{IHC} \mathrm{Ca}^{2+}$ current and $\mathrm{Ca}^{2+}$ indicator fluorescence. For each $\mathrm{Ca}^{2+}$ microdomain, stimuli of different frequencies were presented sequentially (either from $200 \mathrm{~Hz}$ to $2 \mathrm{kHz}$ or from $2 \mathrm{kHz}$ to $200 \mathrm{~Hz}$ ) in an interleaved manner (the "sequence" was repeated 10 times). 
Table 1: Cutoff frequency of IHC voltage response to mechanotransduction-like current

\begin{tabular}{|c|c|c|c|c|c|c|c|c|}
\hline & \multicolumn{8}{|c|}{ Cutoff Frquency, $f_{0}(\mathrm{~Hz})$} \\
\hline Current Amplitude (pA) & \multicolumn{3}{|c|}{200} & \multicolumn{3}{|c|}{500} & \multicolumn{2}{|c|}{1000} \\
\hline Holding Potential (mV) & -77 & -67 & -57 & -77 & -67 & -57 & -77 & -67 \\
\hline ABW26032012_a1e2 & 313.1 & 523.2 & 135.5 & 324.9 & 539.2 & 47.0 & & \\
\hline ABW26032012_a1e3 & 558.1 & 750.7 & 1235.1 & 613.4 & 868.1 & 1116.4 & & \\
\hline ABW26032012_a1e4 & 420.1 & 598.8 & 823.2 & 426.9 & 604.8 & 831.1 & & \\
\hline ABW26032012_a1e5 & 308.1 & 399.9 & 489.7 & 387.9 & 420.5 & 521.5 & & \\
\hline ABW30032012_a1e1 & & & & & & 319.6 & & \\
\hline ABW30032012_a1e2 & 206.2 & & 191.7 & 199.2 & 296.5 & 316.2 & 380.9 & 481.2 \\
\hline ABW30032012_a1e3 & 170.4 & 196.5 & 222.2 & 202.9 & 245.1 & 309.6 & & \\
\hline ABW30032012_a1e4 & 208.9 & 333.3 & 467.8 & 289.7 & 390.0 & 545.5 & 452.4 & \\
\hline Average & 312 & 467 & 509 & 349 & 481 & 527 & 417 & 481 \\
\hline $\begin{array}{l}\text { Standard Deviation, s.d. } \\
(\mathrm{Hz}) .\end{array}$ & 138 & 198 & 398 & 145 & 212 & 356 & - & - \\
\hline Sample Size, $n$ & 7 & 6 & 7 & 7 & 7 & 7 & 2 & 1 \\
\hline Standard Error, s.e.m. (Hz). & 52.1 & 80.9 & 150.4 & 54.6 & 80.2 & 134.7 & - & - \\
\hline
\end{tabular}

Figure 3.5 shows, as an example, the time trace (panel a) and Fast Fourier Transform (FFT, panel b) of membrane potential $\left(\mathrm{V}_{\mathrm{m}}\right), \mathrm{Ca}^{2+}$ current $\left(\mathrm{I}_{\mathrm{Ca}}\right)$ and $\mathrm{Ca}^{2+}$ indicator fluorescence $\left(\Delta \mathrm{F} / \mathrm{F}_{0}\right)$ for a $\mathrm{Ca}^{2+}$ microdomain, which showed modulation of the fluorescence up to $1 \mathrm{kHz}$ of stimulation (arrow in bottom of panel b). The membrane potential was estimated post hoc from a RC circuit model similar to that in Figure 3.3c, to account for the error induced by series resistance $\left(R_{S}\right)$. The reasons of not performing normal online/offline $R_{S}$ compensation methods are that (1) feedback-based $R_{s}$ compensation may introduce oscillation into the system and that (2) currents through $\mathrm{R}_{\mathrm{S}}$ would be dominated by capacitance transients not recorded by the PatchMaster software. 

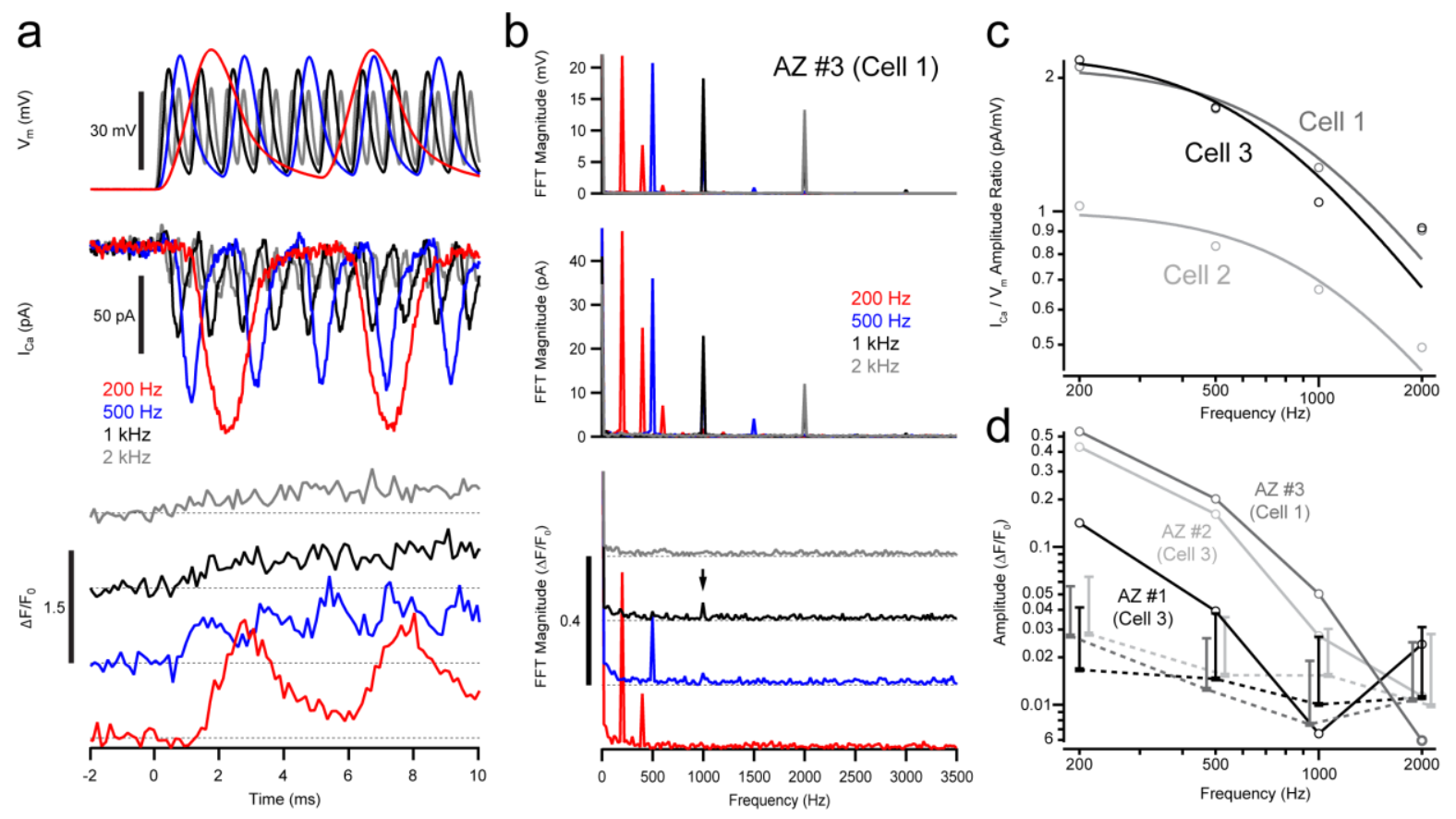

Figure 3.5: Synaptic calcium signal of mature IHCs with oscillating potential.

(a) Oscillating command voltages (upper panel) of different frequencies elicited frequency-modulated $\mathrm{I}_{\mathrm{Ca}}$ (middle panel) in an IHC of a hearing mouse (p15). Peak $\mathrm{I}_{\mathrm{Ca}}$ during each cycle diminished with increasing frequency. Lower panel shows the fluorescence change of Fluo-4FF in a $\mathrm{Ca}^{2+}$ microdomain: a robust oscillation in $\Delta \mathrm{F}$ is observable at $500 \mathrm{~Hz}$. (b) Fast Fourier transform (FFT) of the membrane potential (upper panel), $\mathrm{I}_{\mathrm{Ca}}$ (middle panel) and $\Delta \mathrm{F}$ (lower panel) during the $50 \mathrm{~ms}$ stimulation. For $\Delta \mathrm{F}$, a small peak was detectable even at $1 \mathrm{kHz}$, indicating a $\mathrm{Ca}^{2+}$-microdomain was able to produce oscillation at such frequency. (c) Amplitude of $\mathrm{I}_{\mathrm{Ca}}$ (open circles) estimated as the FFT peak at the stimulus frequency, normalized to attenuation in $\mathrm{V}_{\mathrm{m}}$ predicted by a $\mathrm{RC}$ circuit model. Solid lines are line fits to the same filter function as in Figure 3.4. Each shade of grey represents data from a single IHC. (d) Fluorescence oscillation (open circles and solid lines) of $3 \mathrm{Ca}^{2+}$ microdomains from 2 cells of similar $\mathrm{I}_{\mathrm{Ca}}$ amplitude [Cells 1 and 3 in (e)], estimated as the FFT peak at the stimulus frequency. Dotted lines and error bars represent the mean and dispersion $(3 \times$ s.d.) of background noise in the vicinity $( \pm 100 \mathrm{~Hz})$ of the stimulus frequency, offset horizontally for clarity. In all three microdomains the fluorescence oscillation falls completely to noise level at $2 \mathrm{kHz}$.

As shown in this representative example, the $\mathrm{Ca}^{2+}$ current followed the membrane potential but amplitude of its modulation (a.c. component) progressively declined with increasing frequencies. Attenuation in the $\mathrm{Ca}^{2+}$ current (voltage-clamp) was analyzed by fitting a lowpass filter function to the data (see methods; examples in Figure 3.5c). An average $f_{0}$ of $867 \pm 166 \mathrm{~Hz}(\mathrm{n}=3 \mathrm{IHCs})$ was observed. For $\mathrm{Ca}^{2+}$ currents, the amplitude of FFT at the 
stimulus frequency was normalized to the estimated $\mathrm{V}_{\mathrm{m}}$ (see above). The a.c. component of the $\mathrm{Ca}^{2+}$ indicator fluorescence declined at even lower stimulus frequencies, being often visible at $500 \mathrm{~Hz}\left(5 / 8 \mathrm{Ca}^{2+}\right.$ microdomains) and occasionally detectable at $1 \mathrm{kHz}$ (peak in FFT; $3 / 8 \mathrm{Ca}^{2+}$ microdomains; $8 / 8$ for $500 \mathrm{~Hz}$ ). Figure $3.5 \mathrm{~d}$ compares the magnitude of $\mathrm{Ca}^{2+}$ signal observed at the stimulus frequency and the noise level, which was taken as the average plus three-times the s.d. magnitude around $( \pm 100 \mathrm{~Hz})$ the stimulus frequency. I did not attempt to fit the $\mathrm{Ca}^{2+}$ indicator fluorescence with the filter function as most data points at higher frequencies (1 kHz and $2 \mathrm{kHz}$ ) were below or near background noise level.

\subsubsection{Biophysical properties of calcium current}

Previous work identified that $>90 \%$ of the voltage-gated $\mathrm{Ca}^{2+}$ influx in IHCs before and after the onset of hearing is carried by L-type voltage-gated channels containing the $\alpha 1 \mathrm{D}$ pore forming subunit (Cav1.3 channels; Platzer et al., 2000; Brandt et al., 2003; Dou et al., 2004). However, even with the same $\alpha 1$ subunit, $\mathrm{Ca}^{2+}$ channels are prone to modulation mechanisms including splice isoforms, the presence of different auxiliary subunits and the binding of calmodulin or calcium binding proteins (CaBPs) (Yang et al., 2006; Cui et al., 2007; JuhaszVedres et al., 2011; Tan et al., 2011). Therefore, whole-cell patch-clamp recordings from IHCs were used to study the biophysical properties of the $\mathrm{Ca}^{2+}$ current during postnatal development.

As shown in Figure 3.6a, peak-normalized $\mathrm{Ca}^{2+}$ current-voltage (IV) relationships of p10 and p14-16 IHCs overlapped with indistinguishable half-activation potentials ( $\mathrm{V}_{\text {half }}$, Figure 3.6b; p10: $-29.74 \pm 0.35 \mathrm{mV}$; p14-16: $-29.73 \pm 0.36 \mathrm{mV} \mathrm{p}=0.97$, Student's t-test) as estimated by fitting a Boltzmann function to the fractional activation curves. Despite a subtle difference in their slope (p10: $7.10 \pm 0.09 \mathrm{mV}$; p14-16: $7.39 \pm 0.06 \mathrm{mV} ; \mathrm{p}=0.0049$, Wilcoxon Rank Test), this indicates that the voltage-dependence of activation did not change upon onset of hearing. Inactivation of $\mathrm{Ca}^{2+}$ influx, under highly buffered condition $\left.(10 \mathrm{mM} \text { [EGTA }]_{\mathrm{i}}\right)$, was identical between p10 and p14-16 (Figure 3.6c).

To probe whether the subsequent maturational decline of the $\mathrm{Ca}^{2+}$ current (Fig. 1a) reflects a reduction in the number of channels, single channel current or maximal channel open probability Thomas Frank performed non-stationary fluctuation analysis of $\mathrm{Ca}^{2+}$ tail currents (Roberts et al., 1990; Brandt et al., 2005) in IHCs on postnatal days 9-10, 14-16 and 21-30. 
He found a decrease in the number of $\mathrm{Ca}^{2+}$ channels during development that almost completely accounted for the reduction of the whole-cell $\mathrm{Ca}^{2+}$ current (Figure 3.7a,b). There was no significant change in the estimated single channel current $(\mathrm{p}=0.103$, ANOVA; Figure 3.7c) and only a small reduction in the estimated maximal open probability measured in the presence of the dihydropyridine agonist BayK8644 ( $p<0.001$, ANOVA; Figure 3.7c). These data together suggest that, during postnatal maturation, the number of $\mathrm{Ca}^{2+}$ channels of IHCs decreases while the biophysical properties of their $\mathrm{Ca}^{2+}$ channels remain largely unchanged.
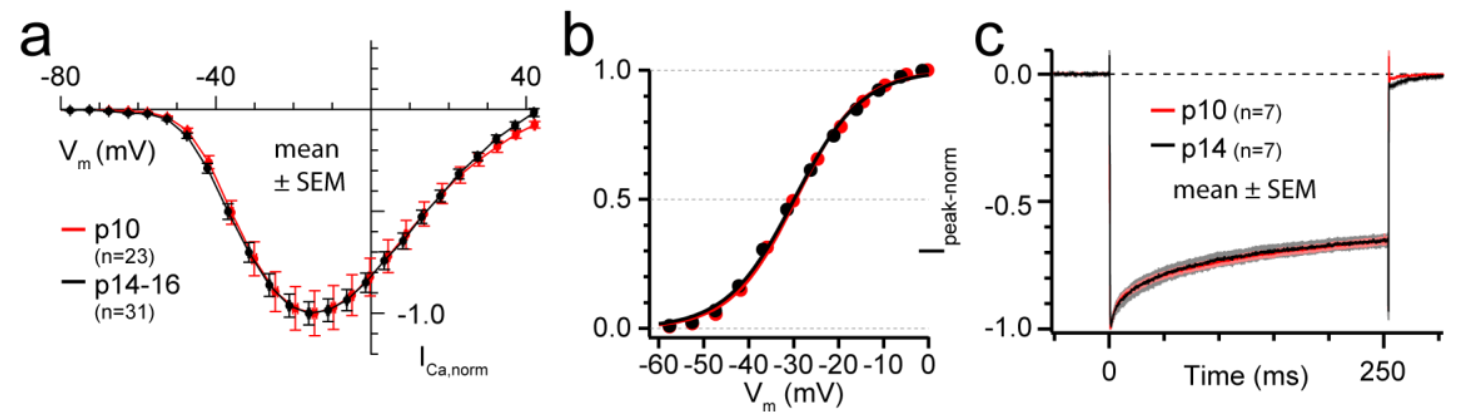

Figure 3.6: Unaltered biophysical properties of $\mathrm{Ca}^{2+}$ current during development.

(a) Average steady-state IV for p10 (red) and p14-16 (black) IHCs in $5 \mathrm{mM}\left[\mathrm{Ca}^{2+}\right]_{\mathrm{e}}$, normalized to peak $\mathrm{I}_{\mathrm{Ca}}$ (for panels a and b: p10, $\mathrm{n}=23 \mathrm{IHCs} ; \mathrm{p} 14-16, \mathrm{n}=31 \mathrm{IHCs}$ ). (b) Activation curve for $\mathrm{I}_{\mathrm{Ca}}$, obtained by dividing the $\mathrm{I}_{\mathrm{Ca}}-\mathrm{V}$ curve in (a) by a line-fit to the linear portion $(-5$ to $+20 \mathrm{mV})$. Solid lines represent fitting of a Boltzmann function in the form of $1 /\left(1+\exp \left(V_{\text {half }}-V /\right.\right.$ slope $)$. (c) Average inactivation of $\mathrm{I}_{\mathrm{Ca}}$ during a $254 \mathrm{~ms}$ depolarization in $5 \mathrm{mM}[\mathrm{Ca}]_{\mathrm{e}}$ and highly buffered $\left(10 \mathrm{mM}[\mathrm{EGTA}]_{\mathrm{i}}\right)$ condition. No obvious difference was observed between the inactivation kinetics of the two age groups ( 6 repetitions per cell; p10, n= 7 IHCs; p14-16, n = 7 IHCs).
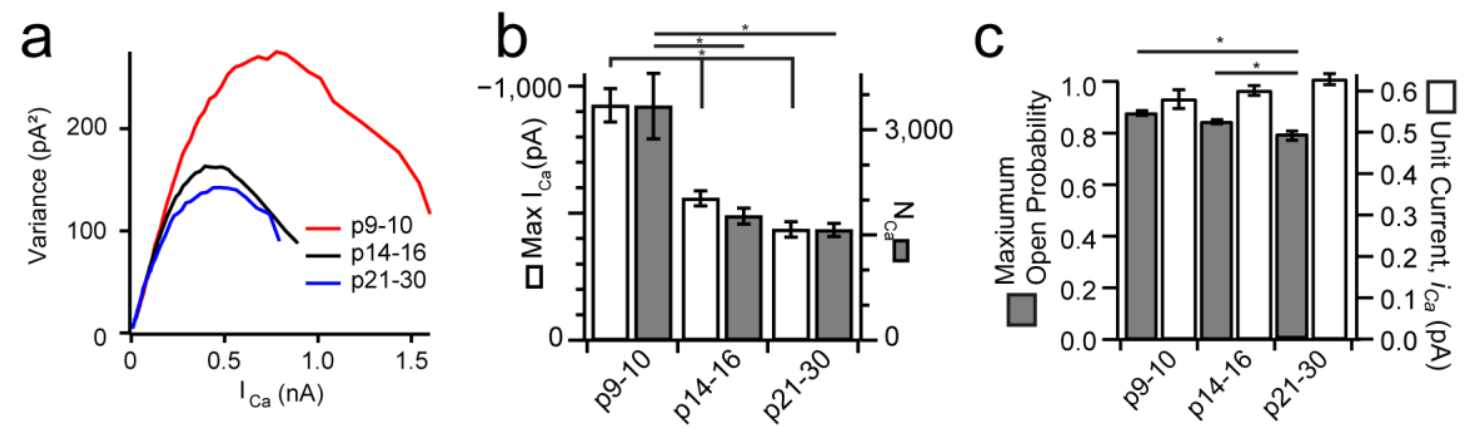

Figure 3.7: Non-stationary fluctuation analysis reveals channel number as major change in development

(a) Grand averages of variance vs. mean relationships in non-stationary fluctuation analysis of $\mathrm{Ca}^{2+}$ tailcurrents. $\left(\left[\mathrm{Ca}^{2+}\right]_{\mathrm{e}}=10 \mathrm{mM} \text {, [BayK8644 }\right]_{\mathrm{e}}=5 \mu \mathrm{M}$; For whole figure: $\mathrm{p} 9-10, \mathrm{n}=13$ IHCs, p14-16, $\mathrm{n}=31$ IHCs, p21-30, $\mathrm{n}=27 \mathrm{IHCs}$ ) (b) The decrease in whole cell $\mathrm{Ca}^{2+}$ current ( $\mathrm{I}_{\mathrm{Ca}}$, empty bars) was largely 
matched by the reduction in number of $\mathrm{Ca}^{2+}$-channel ( $\mathrm{N}_{\mathrm{Ca}}$, solid bars; p9-10, $\mathrm{n}=16$ IHCs; $\mathrm{p} 14-16, \mathrm{n}=33$ IHCs; p21-30, $\mathrm{n}=26 \mathrm{IHCs}$ ) during postnatal development. (c) There was no significant change in the estimated single channel current (in $10 \mathrm{mM}\left[\mathrm{Ca}^{2+}\right]_{e}$, holding potential: $-68 \mathrm{mV}$; p9-10: $0.58 \pm 0.02 \mathrm{pA}$; p14-16: $0.60 \pm 0.01 \mathrm{pA}$; p21-30: $0.63 \pm 0.01 \mathrm{pA}$; ANOVA, $\mathrm{p}=0.103$ ) and only a small reduction in the estimated maximal open probability measured in the presence of the dihdropyridine agonist BayK8644 (p9-10: $0.88 \pm 0.01$; p14-16: $0.84 \pm 0.01$; p21-30: $0.79 \pm 0.02$; Tukey's test for multiple comparison, p910 vs $\mathrm{p} 14-16, \mathrm{p}=0.27$; $\mathrm{p} 9-10$ vs $\mathrm{p} 20-30, \mathrm{p}<0.001 ; \mathrm{p} 14-16$ vs $\mathrm{p} 20-30, \mathrm{p}=0.006$ ).

\subsubsection{Subcellular distribution of calcium channels}

Given that a developmental decrease in $\mathrm{Ca}^{2+}$ channel number was observed (see section 3.1.2), the obvious next step was to investigate where these channel are located. With a combination of immunohistochemistry and live $\mathrm{Ca}^{2+}$ imaging, we showed that the $\mathrm{Ca}^{2+}$ channels are redistributed and progressively confined to active zones during postnatal development (Figure $3.8)$. 


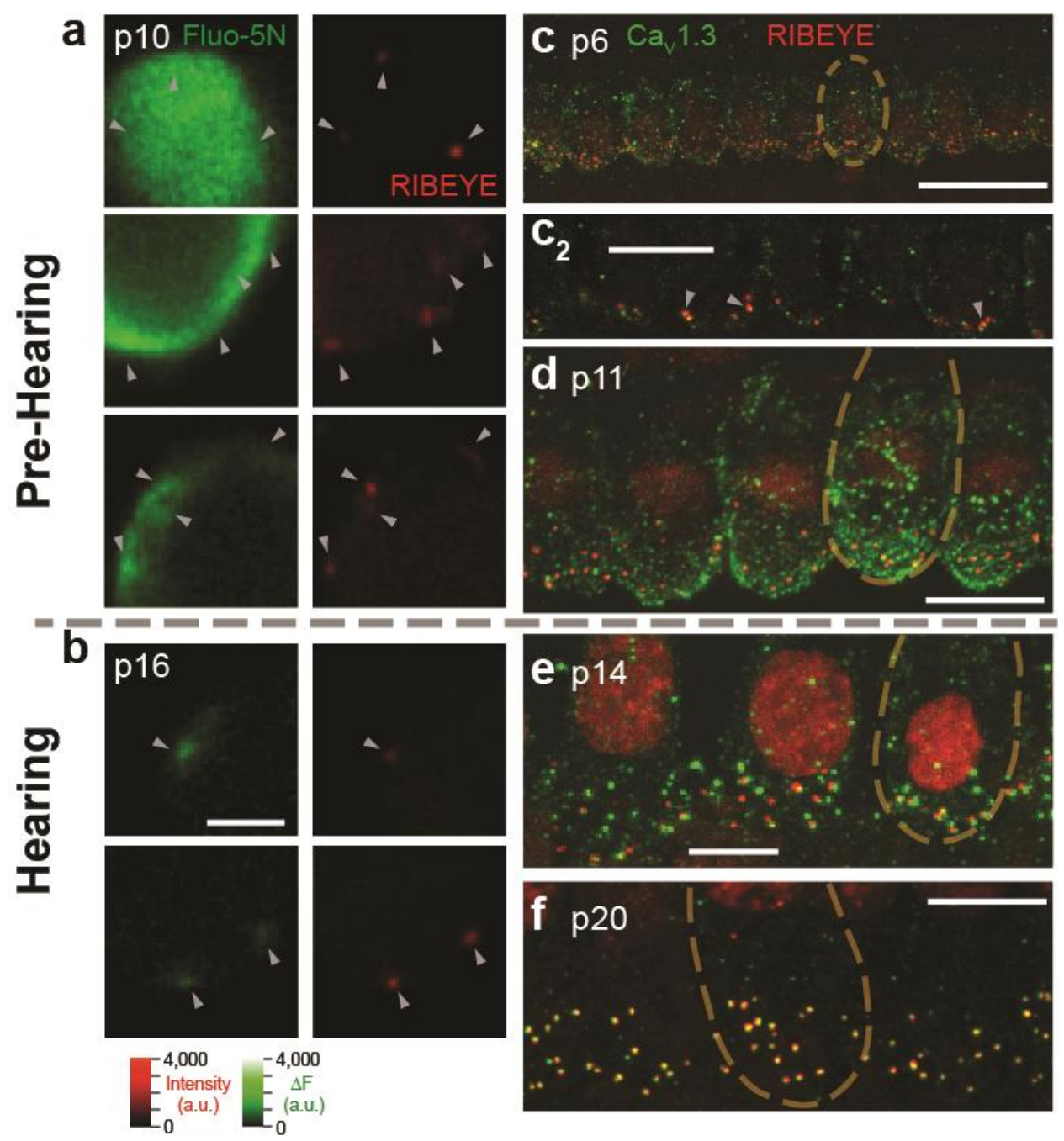

Figure 3.8: Maturational confinement of $\mathrm{Ca}^{2+}$ channel and $\mathrm{Ca}^{2+}$ signal towards active zone revealed by $\mathrm{Ca}^{2+}$ imaging and immunohistochemistry.

(a-b) Examples of XY scans across the basolateral portion of IHCs before (a, p10) and after (b, p14) the onset of hearing. Left and right panels show peptide-labelled synaptic ribbons (position marked with arrowheads in both left and right panels) and increase in Fluo-5N (400 $\mu \mathrm{M})$ fluorescence during a step depolarization to $-7 \mathrm{mV}\left(2 \mathrm{mM}[\mathrm{EGTA}]_{\mathrm{i}}\right)$. Note the relatively widespread sub-membrane $\mathrm{Ca}^{2+}$ signal in immature IHCs as compared to the local $\mathrm{Ca}^{2+}$ rise in mature IHCs. Still, the highest $\left[\mathrm{Ca}^{2+}\right]$ was usually observed at the ribbons-occupied AZs. Scale bar: $3 \mu \mathrm{m}$. (c) At p6, a projection of confocal images shows the basal halves of IHCs in the organ of Corti whole-mount, immunolabeled for $\mathrm{Ca}_{\mathrm{V}} 1.3$ (green) and RIBEYE/CtBP2 (red). Gold dashed lines in c-f demarcate the extent of an IHC. $\mathbf{c}_{2}$ : Single sections show $\mathrm{Ca}_{\mathrm{V}} 1.3$ spots and also more diffuse staining throughout the cell membrane. (d) At p11 puncta of $\mathrm{Ca}_{V} 1.3$ were observed throughout the basolateral membrane, especially the basal pole. (e) By p14 the clusters of $\mathrm{Ca}_{\mathrm{V}} 1.3$ were concentrated in the basal poles near synaptic ribbons, while the entire inner hair cell membrane remained weakly labeled for $\mathrm{Ca}_{\mathrm{V}} 1.3$. (f) At p20 there was very little extrasynaptic labeling and the cell membrane was no longer diffusely immunoreactive for $\mathrm{Ca}_{\mathrm{V}} 1.3$. Each synaptic ribbon was 
accompanied by a cluster of $\mathrm{Ca}_{\mathrm{v}} 1.3$ and only few $\mathrm{Ca}_{\mathrm{v}} 1.3$ clusters remained ribbonless. Images in d-f are maximum intensity projections. Scale bars: c, $20 \mu \mathrm{m} ; \mathrm{c}_{2}$, d, f, $10 \mu \mathrm{m} ; \mathrm{e}, 5 \mu \mathrm{m}$.

Figure 3.8a shows the $\mathrm{Ca}^{2+}$ signal observed when a pre-hearing (p10) IHC was depolarized to $-7 \mathrm{mV}$. In comparison to the spatially-confined $\mathrm{Ca}^{2+}$-microdomains of mature IHCs (Figure $3.8 \mathrm{~b})$, I found a spatially-extended rise of $\left[\mathrm{Ca}^{2+}\right]_{\mathrm{i}}$ in pre-hearing IHCs under the same conditions $\left(400 \mu \mathrm{M}[\text { Fluo- } 5 \mathrm{~N}]_{\mathrm{i}}, 2 \mathrm{mM}[\text { EGTA }]_{\mathrm{i}}, 5 \mathrm{mM}\left[\mathrm{Ca}^{2+}\right]_{\mathrm{e}}\right)$. In many of the pre-hearing IHCs, peak fluorescence change of the $\mathrm{Ca}^{2+}$ indicator was observed at the $\mathrm{AZ}$, marked by a fluorescent RIBEYE binding peptide (Zenisek et al., 2004; Francis et al., 2011) (bottom row in Figure 3.8a, AZs marked by arrowheads), indicating that enrichment of $\mathrm{Ca}^{2+}$-channels at the AZ already occurred before the onset of hearing. This is further supported by immunohistochemistry at p6-11, showing spot-like synaptic Cav1.3-immunofluorescence adjacent to each of the RIBEYE/CtBP2 immunofluorescent spots (arrowheads in Figure $3.8 \mathrm{c}_{2}$ ). However, we also noted Cav1.3 immunoreactivity outside ribbon-occupied AZs of IHCs before the onset of hearing, which often appeared spot-like but could also be diffuse and spread out along the entire basolateral plasma membrane.

I attempted to quantifiy the density of $\mathrm{Ca}_{\mathrm{V}} 1.3$ immunofluorescence at the synaptic region and in at the extrasynaptic plasma membrane. To do so, I identified the ribbon-type AZ with RIBEYE fluorescence and registered the center of each puncta in 3-dimensional (small spheres in Figure 3.9b). Immunofluorescence in the Cav1.3 channel within $400 \mathrm{~nm}$ of the each ribbon center was considered ribbon-associated. Because of difficulty in directly defining the plasma membrane region from the staining, it was approximated by connecting any ribbons within $3 \mu \mathrm{m}$ distance (black lines in Figure 3.9b), which covers around $84 \%$ of the nearest neighbor distances in mature IHCs (calculated from distribution in Meyer et al., 2009), and regarding the fluorescence signal inbetween as membrane-associated (a cylinder of $800 \mathrm{~nm}$ diameter with concave ends; illustrated in Figure 3.9c). I found that at p6 the fluorescence density on the basal plasma membrane was around $43 \%(n=6 \mathrm{IHCs})$ of that of the ribbonoccupied AZ, while at p21 it decreased to only $9 \%(\mathrm{n}=3 \mathrm{IHCs})$. 
a

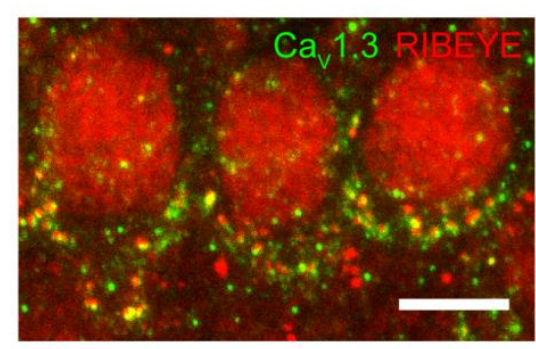

b

C
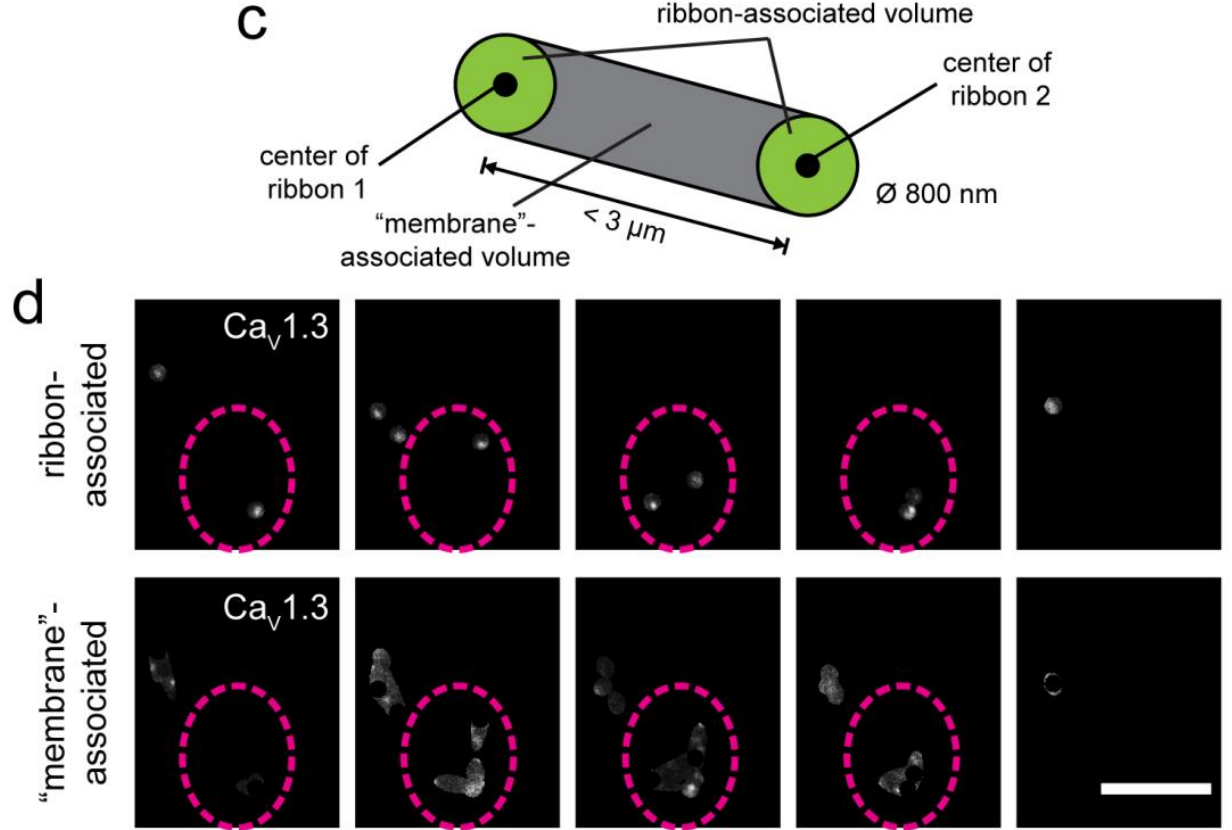

Figure 3.9: Estimation of density of membrane-associated vs ribbon-associated Cav1.3

(a) A projection of an example confocal stack used for analysis (p6). Ca 1.3 staining is shown in green and RIBEYE staining is shown in red. Juxtaposing $\mathrm{Ca}_{\mathrm{V}} 1.3$ and RIBEYE puncta were identified as synapses and the center of the ribbon at each synapse was estimated from 2D Gaussian fit to the confocal section showing brightest fluorescence (in focus). (b) A 3-dimensional representation of identified synaptic ribbons in (a). Large colored spheres represent nuclei and small spheres represent ribbons in the cell with the respective color. Black lines connect ribbons within $3 \mu \mathrm{m}$ distance. (c) Illustration of how ribbon-associated and membrane-associated volumes were defined. (d) Consecutive confocal sections in the $\mathrm{Ca}_{\mathrm{V}} 1.3$ channel masked by either the ribbon-associated (upper panels) or membrane-associated (lower panels) volume. Purple broken ovals mark a clustered of ribbons identifiable in (b).

\subsubsection{Calcium microdomain at active zones}

If enrichment of $\mathrm{Ca}^{2+}$ channel to ribbon-occupied active zones (AZs) already occurred before the onset of hearing, one can restrict the $\mathrm{Ca}^{2+}$ signal to the $\mathrm{AZ}$ by including a high 
concentration of the $\mathrm{Ca}^{2+}$ chelator EGTA into the intracellular solution (Frank et al., 2009). In combination with fluorescent-labeling of presynaptic ribbons (Zenisek et al., 2004; Francis et al., 2011), I studied the synaptic $\mathrm{Ca}^{2+}$ influx at AZs of pre-hearing IHCs, which has been shown to be heterogeneous in mature IHCs (Frank et al., 2009).

AZ-associated $\mathrm{Ca}^{2+}$ microdomains were first identified in an XY scan and co-localization with the fluorescent RIBEYE-binding peptide (see Material and Methods). In all cases when ribbons were brightly labeled by the fluorescent peptide, I only observed $\mathrm{Ca}^{2+}$ microdomains that were associated with a ribbon. Identified $\mathrm{Ca}^{2+}$ microdomains were than further analyzed using either a line-scan (for size and amplitude measurements) or a point-scan (voltage dependence measurements) protocol.

Figure 3.10a shows several examples of the change in fluorescent intensity of $\mathrm{Ca}^{2+}$ indicator at ribbon-occupied AZs under the line-scan protocol (20 ms depolarization to $-7 \mathrm{mV}$ ), presented in pseudocolor. Panel $\mathrm{b}$ shows spatial profiles of the fluorescence change $(\Delta \mathrm{F})$ during the second half of the stimulus, where panel $\mathrm{c}$ shows the temporal profile of the $\Delta \mathrm{F}$ (average of 3 pixels centered at maximum). At first glance, the spatiotemporal properties of the synaptic $\mathrm{Ca}^{2+}$-microdomains of p10 and p14 IHCs elicited were generally comparable (Figure 3.10a-c). To quantify these parameters, a Gaussian function was fitted to each of the spatial profile (see Figure 3.10b) and the full-width of half maximum (FWHM) was used as a measurement of the size of the $\mathrm{Ca}^{2+}$ microdomain. Amplitude was taken as the average $\Delta \mathrm{F}$ or baseline-normalized $\Delta \mathrm{F}\left(\Delta \mathrm{F} / \mathrm{F}_{0}\right)$ during the second half of the stimulus. Onset kinetics was estimated by fitting a single exponential function to the temporal profiles (see Figure 3.10c). Decay kinetics was not assessed as the low signal-to-noise ratio after the end of the depolarization in line scan protocols prevents a good estimate. 


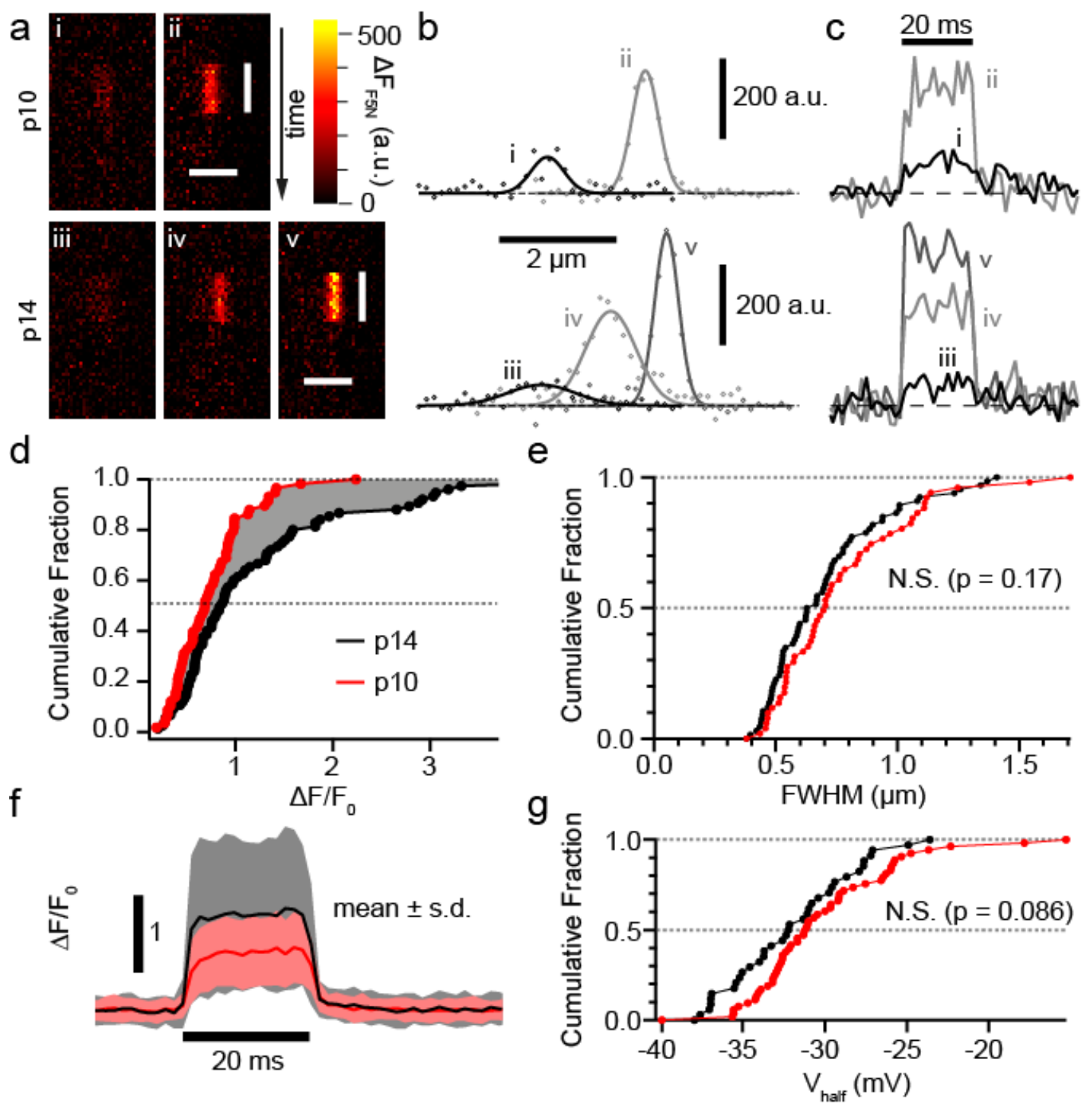

Figure 3.10: Developmental emergence of ribbon-occupied AZs with intense $\mathrm{Ca}^{2+}$ influx

(a) Examples of Fluo-5N fluorescence change $\left(\Delta \mathrm{F}_{\mathrm{F} 5 \mathrm{~N}}\right)$ at synaptic sites upon a $20 \mathrm{~ms}$ depolarization to -7 $\mathrm{mV}$ in pre-hearing (p10) and hearing (p14) IHCs, using line scans. All images were baseline subtracted (average of $50 \mathrm{~ms}$ period before depolarization) and have the same color look-up scale. Vertical bars in (a) and (b) indicate the duration of depolarization. Horizontal scale bars in (a) and (b) are $2 \mu \mathrm{m}$. (b) Profile of $\Delta \mathrm{F}_{\mathrm{F} 5 \mathrm{~N}}$ during the second half of depolarization of examples in (a). Solid lines show Gaussian fits to the line profile, which were used to estimate the spread of $\mathrm{Ca}^{2+}$-signal in (e). (c) Temporal change of Fluo-5N fluorescence at peak position. Roman numbers in (b) and (c) mark the traces corresponding to images in (a). (d) Cumulative distribution of $\Delta \mathrm{F} / \mathrm{F}_{0}$ amplitude in pre-hearing (red trace, $\mathrm{p} 10, \mathrm{n}=58 \mathrm{AZs}$ ) and hearing (black trace, $\mathrm{p} 14-16, \mathrm{n}=75 \mathrm{AZs}$ ) IHCs, showing a selective emergence of high $\mathrm{Ca}^{2+}$ influx synapses (right shift in upper portion of the distributions). (e) Cumulative distribution of full-width-athalf-maximum (FWHM) of $\Delta \mathrm{F}_{\mathrm{F} 5 \mathrm{~N}}$ profiles, estimated by Gaussian fit. No significant difference was found 
between the two age groups. (f) Average profile of $\Delta \mathrm{F} / \mathrm{F}_{0}$ for p10 (red trace, $\mathrm{n}=56$ ) and p14-16 (black trace, $\mathrm{n}=74)$. Shaded bands denote mean \pm s.d.. $(\mathrm{g})$ Distribution of half-activation potential $\left(\mathrm{V}_{\text {half }}\right)$ obtained from fluorescence-voltage measurements (see text).

The first striking result is that the mean $\Delta \mathrm{F} / \mathrm{F}_{0}$ (Figure 3.10f, $\mathrm{p}=0.007$, Wilcoxon Rank Test) amplitudes of mature AZs were significantly greater than those of immature AZs in this data set. This trend is opposite to that observed in whole-cell $\mathrm{Ca}^{2+}$ current (see section 3.1.2 and Figure 3.7). The onset kinetics was significantly slower in $\mathrm{p} 10 \mathrm{Ca}^{2+}$ microdomains $\left(\tau=2.3 \pm 0.3 \mathrm{~ms}, \mathrm{n}=48\right.$ vs $1.1 \pm 0.1 \mathrm{~ms}$ at $\mathrm{p} 14-16, \mathrm{n}=55 ; \mathrm{p}=7.0 \times 10^{-5}$, Wilcoxon Rank Test). Moreover, a broader range of $\Delta \mathrm{F}$ amplitudes in mature IHCs (Figure 3.10a-d) with a significant difference in dispersion between the two datasets was observed ( $\mathrm{p} 10: \mathrm{CV}=0.52$, $\mathrm{n}=58 ; \mathrm{p} 14-16: \mathrm{CV}=0.82, \mathrm{n}=75$; modified Levene's test using median [Brown and Forsythe, 1974], $\mathrm{p}=0.0018)$. Interestingly, the greatest difference occurs at the upper half of the distribution, indicating a selective emergence of AZs with stronger $\mathrm{Ca}^{2+}$ signal in more mature preparations. Their amplitude seemed to further increase during the $3^{\text {rd }}$ postnatal week (Figure 3.12 with previously published datasets from Frank et al., 2009, 2010). However, no significant difference in dispersion was found between the median-normalized p20-31 group (Frank et al., 2010) and either of the p14 groups (new data and Frank et al., 2009).

No significant difference was found between the mean FWHM of p10 and p14-16 groups ( $p=0.17$, Wilcoxon Rank Test). In addition, I tested the voltage-dependence of the $\mathrm{Ca}^{2+}$ signals using the $\mathrm{Ca}^{2+}$ indicator Fluo-4FF (see Material and Methods, p.16), and found no significant difference in $\mathrm{V}_{\text {half }}$ (Figure 3.10g and Figure 3.11c; $\mathrm{p}=0.08$, Wilcoxon Rank Test) or slope ( $p=0.12$, Wilcoxon Rank Test) in the fluorescence-voltage $(F V)$ relationships.

I observed a negative correlation between the amplitude and FWHM of $\mathrm{Ca}^{2+}$ signal Figure 3.11a) but no significant correlation between amplitude and $\mathrm{V}_{\text {half }}$ (Figure 3.11b). I also compared the $\mathrm{V}_{\text {half }}$ estimated from FV and IV (Figure 3.11c) and found indication of positive correlation in both age groups ( $\mathrm{p} 10: \mathrm{r}=0.47, \mathrm{p}<0.001$; $\mathrm{p} 14-16: \mathrm{r}=0.31, \mathrm{p}=0.07$ )

Quantifying peptide-labeled ribbons in whole-cell experiments (Figure 3.8a-b), I found a developmental reduction in number of detectable ribbons from $14.4 \pm 0.7$ at $\mathrm{p} 10(\mathrm{n}=12 \mathrm{IHCs})$ to $11.8 \pm 0.5$ at p14 ( $\mathrm{n}=6 \mathrm{IHCs}$ ) in IHCs of the apical cochlea (approximately 300-1000 $\mu \mathrm{m}$ 
from apex). Analysis of juxtaposed RIBEYE and CaV1.3 immunofluorescence spots also revealed a developmental reduction of ribbon-occupied AZs from $21.1 \pm 1.2$ at p6 $(\mathrm{n}=18$ IHCs) to $12.4 \pm 0.5$ at $\mathrm{p} 9$ ( $\mathrm{n}=15 \mathrm{IHCs})$ to $10.3 \pm 0.4$ at $\mathrm{p} 14(\mathrm{n}=25 \mathrm{IHCs})$ and $9.5 \pm 0.4$ at p21 ( $\mathrm{n}=22 \mathrm{IHCs})$. As an estimate of the total amount of synaptic $\mathrm{Ca}^{2+}$ influx, I multiplied the average $\Delta \mathrm{F}$ amplitude and ribbon number at p10 and p14. Interestingly, the two estimates closely matched with one another (p10: 134 a.u. $\times 14.4=1937$ a.u.; p14: 161 a.u. $\times 11.8=$ 1901 a.u.). Therefore, despite the massive reduction of IHC $\mathrm{Ca}^{2+}$ current during postnatal maturation, IHCs seem to conserve the cumulative $\mathrm{Ca}^{2+}$ influx across all ribbon-occupied AZs.
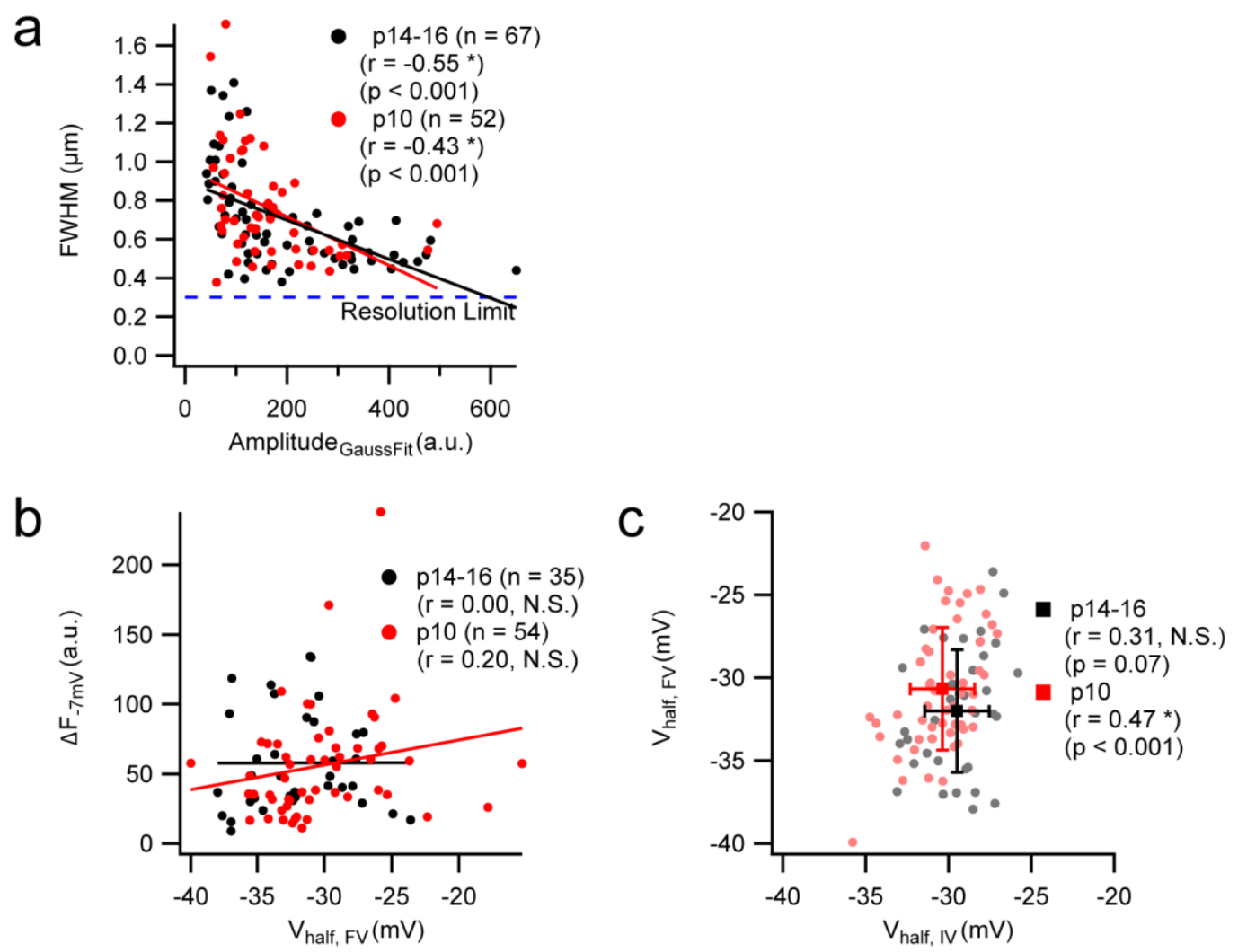

Figure 3.11: Correlation among parameters of $\mathrm{Ca}^{2+}$ microdomain

(a) Negative correlation was observed between FWHM of $\mathrm{Ca}^{2+}$ microdomain and their amplitude. (b) No significant correlation was observed between amplitude of fluorescence change $(\Delta \mathrm{F})$ at $-7 \mathrm{mV}$ and halfactivation potential $\left(\mathrm{V}_{\text {half, FV }}\right)$ in fluorescence-voltage relationship measurements. (c) Half-activation potential in FV and concomitantly measured IV ( $\mathrm{V}_{\text {half, IV }}$ ) showed trends of positive correlation in both p10 and p14-16 age groups. 


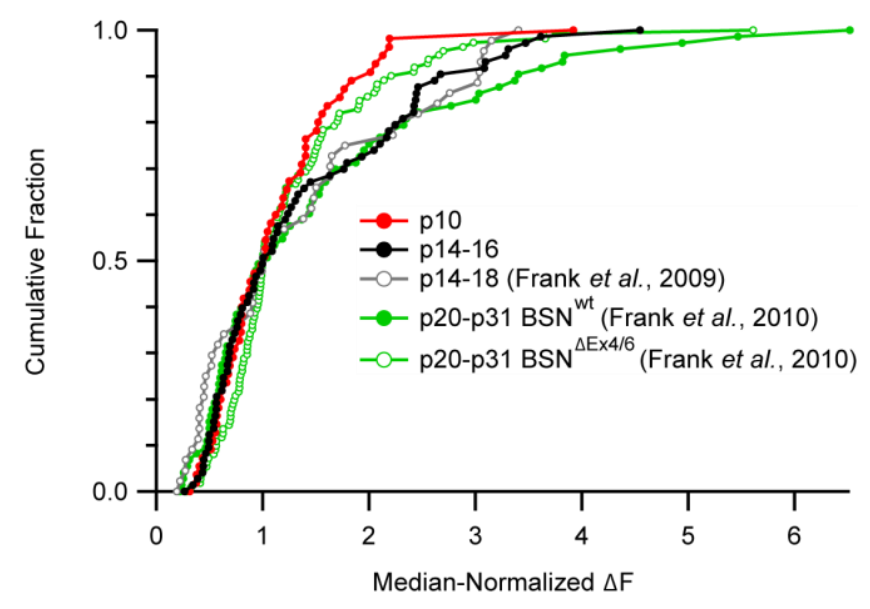

\section{Figure 3.12: Distribution of $\mathrm{Ca}^{2+}$ microdomain amplitude compared with published datasets.}

Cumulative distribution of $\Delta \mathrm{F}$ amplitudes normalized to their median. Figure includes data from wildtype of different age groups, taken from this study (p10, red; p14-16, black) and previous publications as indicated (Frank et al., 2009, 2010). Data from a mutant of presynaptic scaffold Bassoon (also from Frank et al., 2010) is included for comparison.

\subsubsection{Concomitant changes in synaptic morphology}

With the help of Mark A. Rutherford, I looked at the distribution of several pre- and postsynaptic markers in organ of Corti whole mount preparations at various developmental stages. We labeled IHCs for AZ markers including RIBEYE and Bassoon, a scaffold protein contributing to ribbon anchorage (Khimich et al., 2005). We also labeled the postsynaptic density (PSD) with antibodies specific to either AMPA receptor subunit GluA2 only, or both GluA2 and GluA3. Afferent synapses of pre-hearing IHCs displayed several small pairs of AZs and PSDs that were often organized into circular structures greater than $1 \mu \mathrm{m}$ in diameter (Figure 3.13a) larger than the size of their ring-like AMPA receptor clusters in mature synaptic contacts $(<1 \mu \mathrm{m}$, Meyer et al., 2009). Some of the Bassoon-labeled AZs seemed to lack detectable RIBEYE immunofluorescence and likely represented ribbonless AZs. Visualization of the postsynaptic membrane by immunolabeling for the $\mathrm{Na}^{+} / \mathrm{K}^{+}$-ATPase $\alpha 3$ subunit, which specifically labels SGN in the organ of Corti (McLean et al., 2009), suggested that each of these circular structures may correspond to a synaptic contact formed by a single SGN neurite (Figure 3.13d). The contacts became spatially confined, eventually being comprised of one ribbon-occupied AZ and a single large PSD in mature preparations (Figure $3.13 b, c$ and e). Different from the mature synapse, where typically a single ribbon faces the 
center of the ring-like singular AMPA receptor cluster (Meyer et al., 2009; also see Figure $3.13 \mathrm{e})$, the ribbons of the immature synapse were located in its periphery, each juxtaposed with one of the several pre- and postsynaptic specializations (Figure 3.13a, d).

Similar finding was observed with electron microscopy performed in collaboration with Carolin Wichmann. Figure 3.14a shows representative electron micrographs illustrating the spatially extended synaptic contacts of pre-hearing IHCs. Multiple pairs of pre- and postsynaptic densities were observed, both ribbonless and ribbon- occupied (magenta arrowheads in Figure 3.14a). This is also illustrated by a 3D-reconstruction of an immature synapse from serial ultrathin sections (Figure 3.14c) showing 3 ribbon-occupied AZs and at least one small ribbonless AZ with vesicles (arrowhead). The immature ribbons typically assumed a spherical shape (Figure 3.14a, d) and were anchored by two rootlets, as previously described (Sobkowicz et al., 1982). After the onset of hearing the synaptic ribbon usually displayed a droplet- or wedge-like shape (Fig. 4b, d) and was anchored to the AZ along its entire long axis (Figure 3.14b, c). There was an increase in size of the ribbon, the number of ribbon-tethered vesicles per section, and the total length of the PSD with age (summarized in Table 2). 


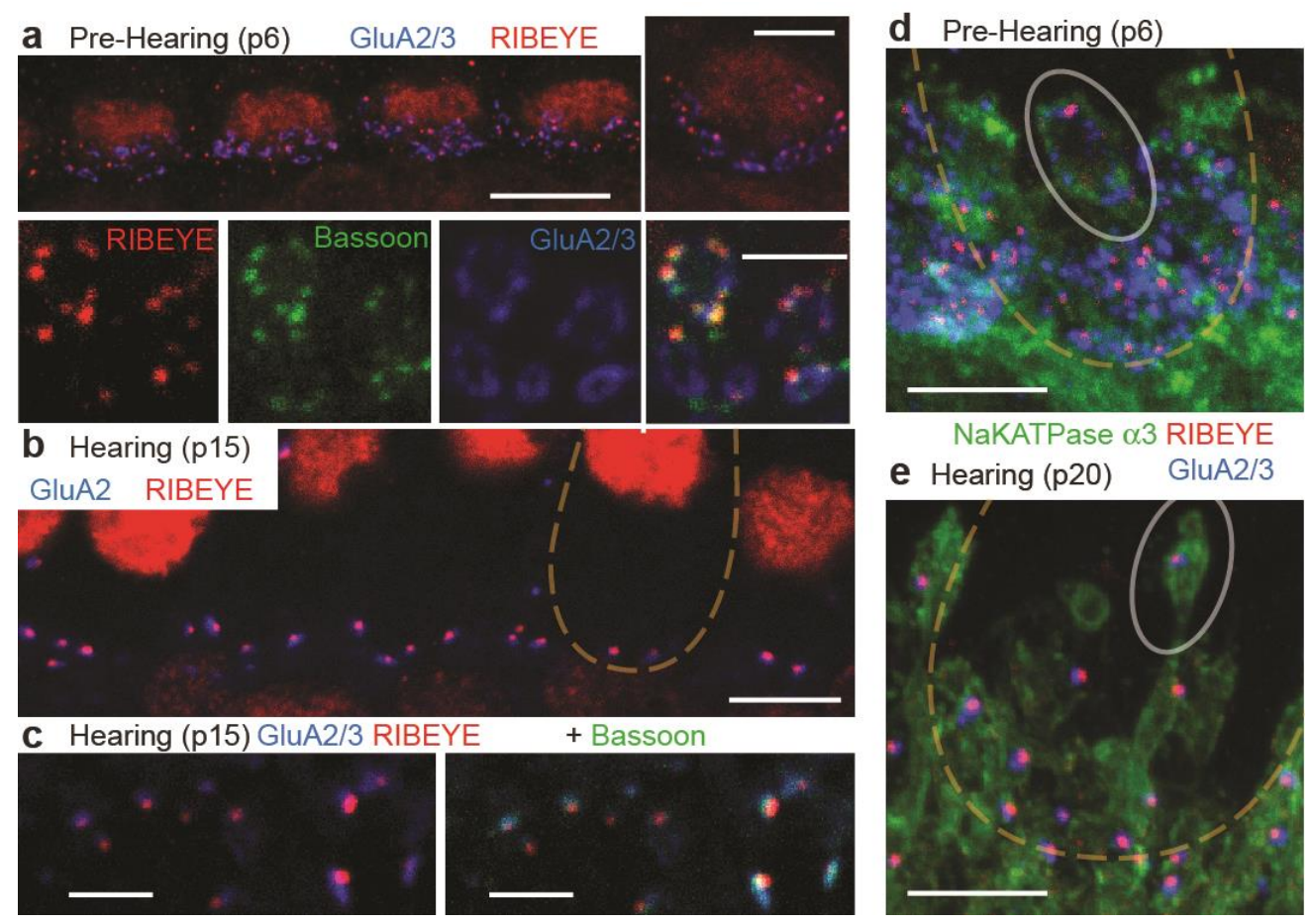

\section{Figure 3.13: Developmental changes in morphology of hair cell afferent synapses}

(a) Upper: At p6, the basal pole of each IHC was contacted by several patches of postsynaptic glutamate receptors (GluA2/3, blue) forming a circular structure. Single confocal section, enlarged at right. Lower: Each GluA2/3 spot was juxtaposed to an AZ spot, marked by protein Bassoon (green) and in some cases by a ribbon (RIBEYE/CtBP2, red). One complex circular structure was outlined in gray in the composite image. Scale bars: upper left, $10 \mu \mathrm{m}$; upper right, $5 \mu \mathrm{m}$; lower panels, $1 \mu \mathrm{m}$ (b) Single confocal section at p15 showing juxtaposed immunofluorescence for RIBEYE/CtBP2 (red) and GluA2 (blue). Scale bar: 5 $\mu \mathrm{m}$ (c) Single confocal section with same antibody combination as in (a) at p15. The individual GluA spots seemed to have contracted into a single ring-like cluster, juxtaposed to a single ribbon. Gold dashed lines in panels $\mathrm{c}$ to e delineate the outline of IHCs. Scale bars: $1 \mu \mathrm{m}$ (d) Projection of confocal images showing GluA2/3 (blue), RIBEYE/CtBP2 (red), and $\mathrm{Na}^{+} / \mathrm{K}^{+} \mathrm{ATPase} \alpha 3$ subunit (green) at $\mathrm{p} 6$. The latter antibody labels the membranes of SGNs, each of which terminates onto the IHC with a bouton-type contact. The large circular structures formed by GluA spots partially encompass the perimeter of the bouton contacts (e.g. highlighted by gray oval). Scale bar: $5 \mu \mathrm{m}$ e: At p20, each postsynaptic bouton (e.g. gray oval) contains only a single condensed glutamate receptor cluster that is juxtaposed to a single presynaptic ribbon. Scale bar: $5 \mu \mathrm{m}$. 


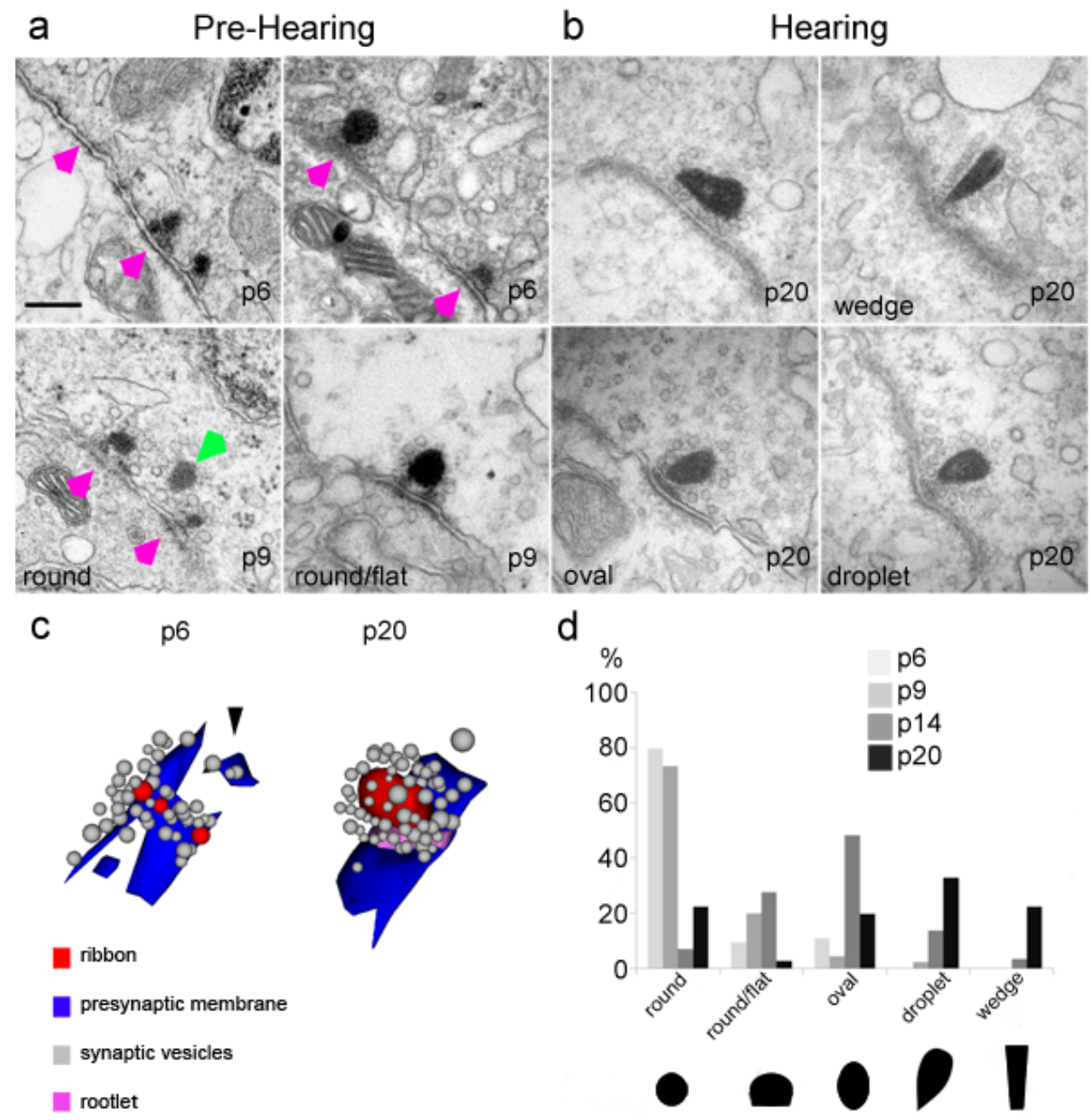

\section{Figure 3.14: Ultrastructural changes of IHC ribbon synapses in development}

(a-b) Representative electron micrographs of IHC ribbon synapses in pre-hearing (a) and hearing (b) mice. (a) Before the onset of hearing, at $\mathrm{p} 6$ and p9, multiple appositions of small discontinuous pre- and postsynaptic densities were found (magenta arrowheads) typically accompanied by one or more roundshaped ribbon(s) anchored close to the presynaptic density. In some cases ribbons were "floating" in the cytoplasm at a greater distance from the membrane (green arrowhead). (b) After the onset of hearing, at p20, typically one ribbon occupied the presynaptic cytoplasm at the AZ. In comparison to before the onset of hearing, the mature pre- and postsynaptic densities were relatively extended and continuous. The micrograph on the upper left is a section that was cut parallel to the long-axis of a ribbon. The other sections were cut parallel to the ribbons' short axes, the perspective from which ribbon-shape was measured. (c) 3D reconstructions from serial sections before (left, p6) and after (right, p20) the onset of hearing. Presynaptic rootlet: density connecting the ribbon to the plasma membrane. (d) Through development the ribbon-shape changed from predominantly round to shapes like oval, droplet-like, or wedge-like, as shown in panels a and b. Scale bar of $200 \mathrm{~nm}$ in panel a applies to panels a-c. 
Table 2: Quantification of ultrastructural properties of developing IHC ribbon synapses

\begin{tabular}{|c|c|c|c|}
\hline & $\begin{array}{l}\text { PSD length } \\
(\mathbf{n m})\end{array}$ & $\begin{array}{l}\text { Ribbon size }^{\mathrm{a}} \\
\quad(\mathbf{n m})\end{array}$ & $\begin{array}{l}\text { Ribbon-associated }^{\mathrm{b}} \\
\text { vesicles per slice }\end{array}$ \\
\hline p6 & $433 \pm 30$ & $106 \pm 4$ & $6.6 \pm 0.4$ \\
\hline $\mathrm{n}_{\mathrm{AZ}}$ & 40 & 40 & 64 \\
\hline p-value (p6 vs p9) & 0.8187 & 0.03897 & 0.5582 \\
\hline p9 & $421 \pm 43$ & $121 \pm 6$ & $6.2 \pm 0.5$ \\
\hline $\mathrm{n}_{\mathrm{AZ}}$ & 35 & 35 & 45 \\
\hline p-value (p9 vs p14) & 0.000384 & 0.0023 & $2.49 \mathrm{E}-05$ \\
\hline p14 & $668 \pm 50$ & $106 \pm 12$ & $10 \pm 0.8$ \\
\hline $\mathrm{n}_{\mathrm{AZ}}$ & 26 & 26 & 29 \\
\hline p-value (p14 vs p20) & 0.26943 & 0.45436 & 0.01063 \\
\hline p20 & $614 \pm 23$ & $169 \pm 6$ & $12.4 \pm 0.5$ \\
\hline $\mathrm{n}_{\mathrm{AZ}}$ & 72 & 76 & 76 \\
\hline \multicolumn{4}{|c|}{$\begin{array}{l}\text { Notes: }{ }^{\text {a }} \text { measured as the length across the longest axis of the synaptic density. } \\
\text { b vesicles within a vesicle diameter distance from the ribbon surface. }\end{array}$} \\
\hline
\end{tabular}

\section{2 $\mathrm{Ca}^{2+}$ dependence of exocytosis in developing IHC}

It has been reported that during development, transmitter release of IHCs changes from a supralinear relationship to a linear relationship with respect to membrane potential (Johnson et al., 2005). However, controversy remains whether this is due to a switch in $\mathrm{Ca}^{2+}$ sensor as suggested by Johnson and colleagues (2010), or a difference in channel-vesicle coupling as demonstrated in the Calyx of Held (Fedchyshyn and Wang, 2005; Wang et al., 2008). Here, I attempted to generate insight by investigating (1) the intrinsic $\mathrm{Ca}^{2+}$ dependence of vesicle release, and (2) how different manipulation of $\mathrm{Ca}^{2+}$ influx affects release (apparent $\mathrm{Ca}^{2+}$ dependence), in both pre-hearing and mature IHCs..

\subsubsection{Probing intrinsic $\mathrm{Ca}^{2+}$-dependence of exocytosis through $\mathrm{Ca}^{2+}$ uncaging}

To look at the intrinsic $\mathrm{Ca}^{2+}$-dependence of exocytosis, I, together with Tina Pangršič, performed UV-laser photolysis of caged $\mathrm{Ca}^{2+}$ (DM-nitrophen) in IHCs of p6-8 and p14-18 IHCs. This method induces spatially homogenous elevations of cytosolic $\left[\mathrm{Ca}^{2+}\right]$ to various levels, which elicits rapid exocytosis at different preparations (Heidelberger et al., 1994; Bollmann et al., 2000; Schneggenburger and Neher, 2000; Beutner et al., 2001). During the experiment, we measured changes in membrane capacitance $\left(\Delta \mathrm{C}_{\mathrm{m}}\right)$ and fluorescence of a low- 
affinity ratiometric $\mathrm{Ca}^{2+}$-indicator (mag-fura-2) upon UV flash, giving us an estimation of vesicle fusion and post-flash cytosolic $\left[\mathrm{Ca}^{2+}\right]$, respectively. A double exponential function with variable delay (see p19, Material and Methods) was used to fit the change in $C_{m}$, from which rate constants and response delay were extracted. In cases where the rate constants of the two components differed by less than a factor of four (4), the traces were re-fitted with a single exponential function. The $\mathrm{C}_{\mathrm{m}}$ rise of approximately half (22 out of 40) of p6-8 IHCs and nearly all (32 out of 33) p14-18 IHCs showed a fast and a slow component. The total amplitude (results of fit) of the $\mathrm{C}_{\mathrm{m}}$ rise was largely independent of $\left[\mathrm{Ca}^{2+}\right]_{\mathrm{i}}$ (Figure 3.16b; linear regression: $r=0.03, p=0.84$, after exclusion of an outlier), as it is the case in $\mathrm{p} 14-25$ IHCs (Beutner et al., 2001; and Figure 3.16b, $r=-0.12, p=0.49$ ). However, it was approximately 3.6 times smaller than at p14-18 $(324 \pm 24 \mathrm{fF} ; \mathrm{n}=40$ vs. $1161 \pm 104 \mathrm{fF} ; \mathrm{n}=$ 31). Moreover, when two kinetically distinct components were present, we found a greater contribution of the slow component in p6-8 IHCs (69\%), while both components contributed equally (49\% slow component) in p14-18 IHCs of the current dataset. Beutner and colleague (2001) reported an average of 30\% contribution from the slow component in p14-25 IHCs.

We first compared the kinetics of exocytic responses with two exponents. As shown in Figure 3.16a, the rate constants of the first responses in p6-8 IHCs (filled red circles) largely overlap with those of p14-17 IHCs (filled black circles), indicating limited, if any, change in $\mathrm{Ca}^{2+}$ dependent kinetics of vesicle fusion. Rate constants from previously published data (Beutner et al., 2001; Pangršič et al., 2010) were overlaid as open circles for comparison. Interestingly, the rate constants of p6-8 IHCs fitted with a single exponential function (pink rhombi) overlap well with those of the second component in both p6-8 and p14-17 IHCs (red and black rhombi, respectively). This may suggest that IHCs with a single-exponential response lack a fast component of release. The reason is unclear and possible explanations will be discussed later. 

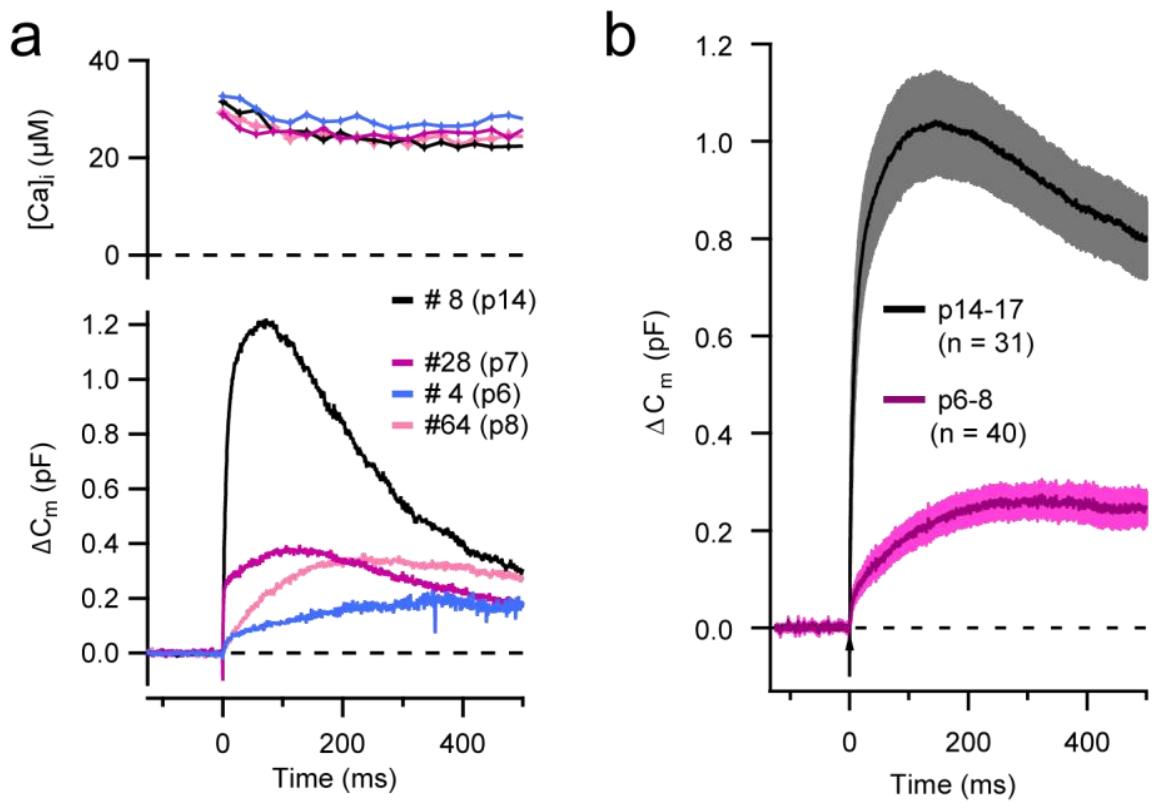

Figure 3.15: Capacitance response of $\mathrm{IHC}$ upon $\mathrm{Ca}^{2+}$ uncaging before and after onset of hearing

(a) Examples of responses of IHCs before and after hearing onset upon UV-flash, with similar post-flash $\left[\mathrm{Ca}^{2+}\right]_{i}$. All capacitance change $\left(\Delta \mathrm{C}_{\mathrm{m}}\right)$ in these examples except \#64 (pink) can be fitted with a double exponential function. (b) Average $\Delta \mathrm{C}_{\mathrm{m}}$ of pre-hearing (p6-8, magenta) and hearing (p14-17, black) IHCs upon UV-flash. Shaded areas represent mean \pm s.d.. Mean post-flash $\left[\mathrm{Ca}^{2+}\right]_{\mathrm{i}}$ : p6-8 $=28.5 \pm 2.3 \mu \mathrm{M}$; p14$17=24.0 \pm 1.4 \mu \mathrm{M}$ (N.S. $\mathrm{p}=0.11)$. 

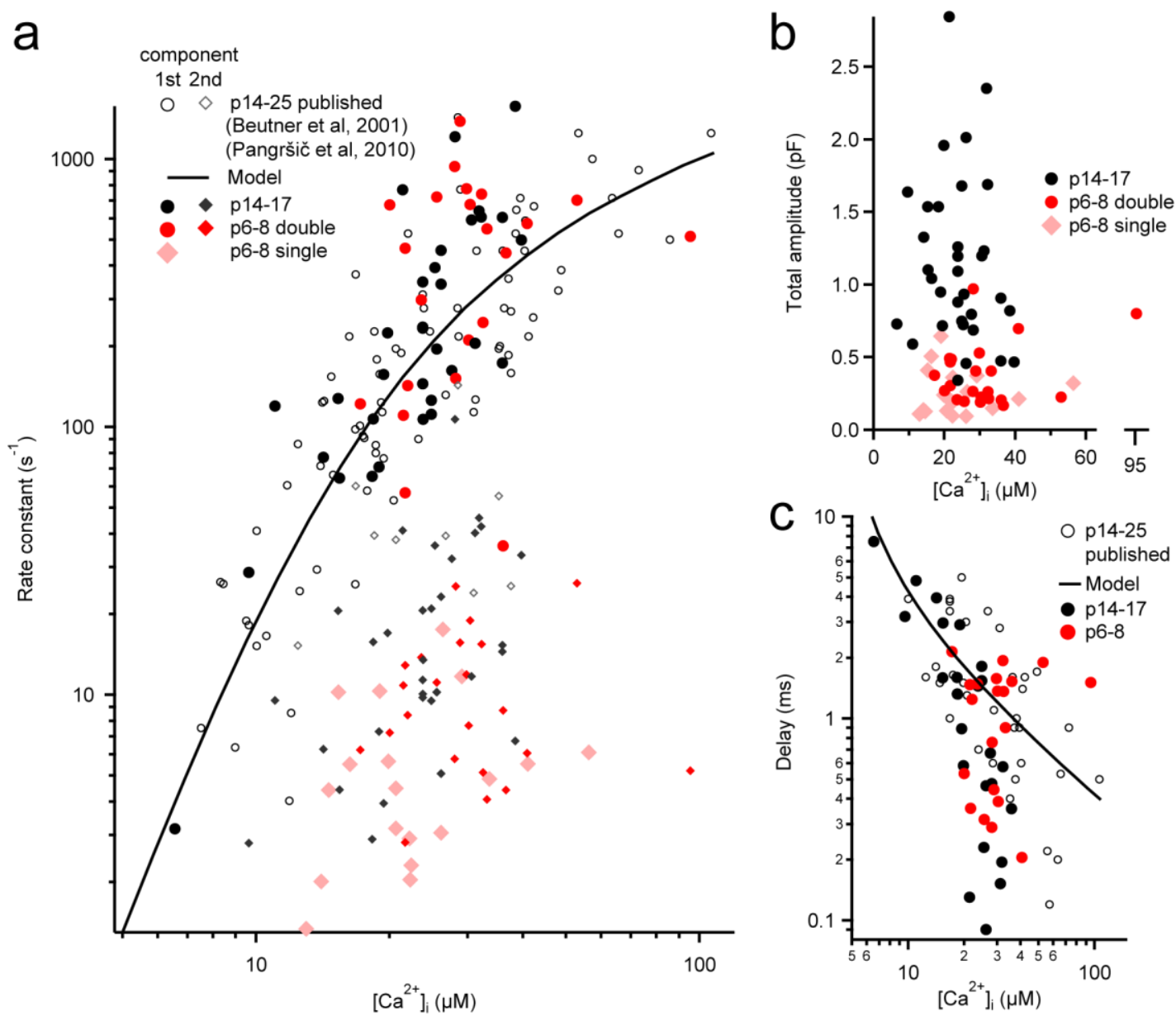

Figure 3.16: $\mathrm{Ca}^{2+}$-dependence of uncaging responses

(a) Rate constants from exponential fits to the capacitance response upon flash photolysis. Circles mark the rate constants for first (fast) component and rhombi mark those for the second (slow) components. Rates of responses in p6-8 IHCs which can only be fitted with a single component are also marked with rhombi. Note the overlap between pre-hearing (p6-8) and hearing (p14+) age groups. (b) Total response amplitudes (sum of first and second component, in case of double exponential fits) were largely independent of post-flash $\left[\mathrm{Ca}^{2+}\right]_{\mathrm{i}}$. (c) Delay of responses vs post-flash $\left[\mathrm{Ca}^{2+}\right]_{\mathrm{i}}$. See Material and Methods for definitions. Solid lines in (a) and (c) are model fits from a previous publication (Beutner et al., 2001).

\subsubsection{Mathematical analysis of binding kinetics}

A five-site kinetic model was previously proposed to explain the observed flash responses in IHC (Beutner et al., 2001), and parameters concerning binding and fusion kinetics were extracted. Here, I attempted to re-implement the 5-site kinetic model described by Beutner and colleagues (2001). Intriguingly, while the simulated rate constants and time to peak release were successfully reproduced using the same parameters, the delay estimates (red 
dashed line, Figure 3.17b) did not match that from the published article (black lines, Figure 3.17). Instead, the published data fits perfectly if delay was estimated as the time till $2 \%$ of available vesicles are released (green dashed line, Figure 3.17b). Thus, I optimized the parameters again with data from experiments performed under identical conditions (Beutner et al., 2001; Pangršič et al., 2010), using the downhill-simplex method (modified from Press et al., 1992 p.408-412, see Material and Methods for details). The new parameters (blue lines, Figure 3.17) improved in capturing the $\mathrm{Ca}^{2+}$ dependence of delay over the old parameters. However, the maximal release rate at high $\left[\mathrm{Ca}^{2+}\right]$ was limited by the lower fusion rate $\gamma$ $\left(792 \mathrm{~s}^{-1}\right)$ at the last step, which does not capture the further increase of rate constant especially apparent for the new dataset (black circles in Figure 3.16). The new datasets were not fitted by the model due to the limited number of datapoints at the lower $\left[\mathrm{Ca}^{2+}\right]$ range. Further considerations needs to be done, for example, the use of alternative models (Lou et al., 2005; Sun et al., 2007) and different experimental procedures (e.g. short laser pulse vs. arc lamp, sampling rate of capacitance measurement).

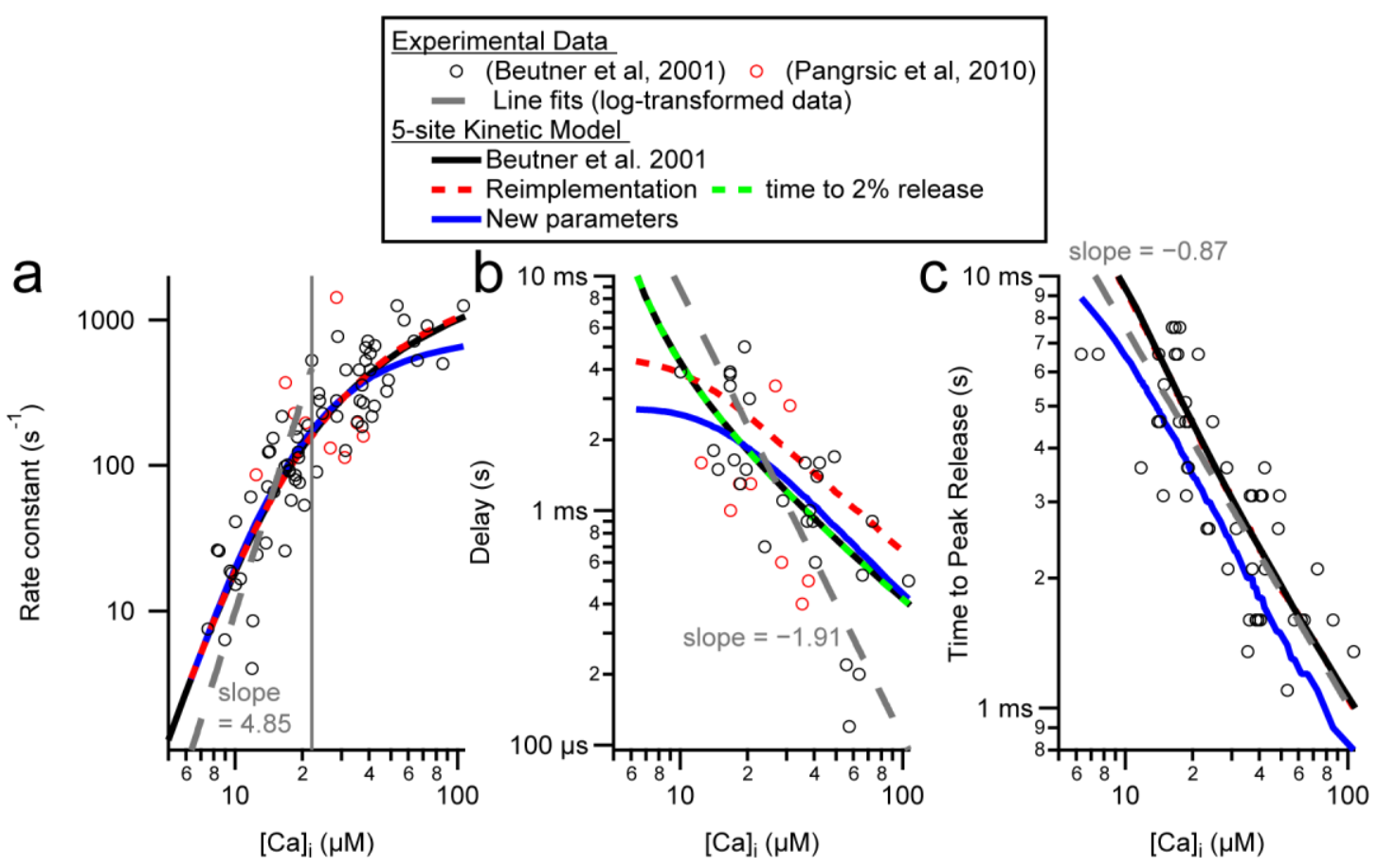

Figure 3.17: Re-implementation of a minimal kinetic model.

Log-log plots of (a) rate constant, (b) response delay, and (c) time to peak release vs post-flash $\left[\mathrm{Ca}^{2+}\right]_{\mathrm{i}}$ from published datasets (Beutner et al., 2001; Pangršič et al., 2010). Overlaid are results from kinetic models implemented: published model (Beutner et al., 2001; black), reimplementation with same 
parameters (red and green dashed lines) and new parameters (blue solid lines). New parameters: $\mathrm{k}_{\mathrm{on}}=50.9 \mu \mathrm{M}^{-1} \mathrm{~s}^{-1}, \mathrm{k}_{\text {off }}=3705 \mathrm{~s}^{-1}, \mathrm{~b}=0.44, \gamma=792 \mathrm{~s}^{-1}$. Grey dashed lines are line fits to data points using orthogonal distance regression. Line in (a) was fitted with first half of the datapoints to avoid saturating region at high $\left[\mathrm{Ca}^{2+}\right]$.

\subsubsection{Apparent cooperativity}

Since no apparent discrepancy was observed in the intrinsic $\mathrm{Ca}^{2+}$ dependence of vesicle release, I proceeded with experiments concerning the coupling of $\mathrm{Ca}^{2+}$ influx to vesicles. Here, I investigated the "apparent cooperativity" of release by measuring the amount of exocytosis of pre-hearing and mature IHCs under different manipulations of $\mathrm{Ca}^{2+}$ influx (Brandt et al., 2005). These different manipulations combined allow us to distinguish between two model of coupling between vesicle and $\mathrm{Ca}^{2+}$ channel, namely " $\mathrm{Ca}^{2+}$-microdomain" and " $\mathrm{Ca}^{2+}$ nanodomain" (for detailed explanation, see Figure 4.2 and section 4.2.1).

Figure 3.18a-b illustrates a typical experiment. A p7 IHC was voltage-clamped under perforated patch configuration achieved by including $250 \mu \mathrm{g} / \mathrm{ml}$ amphotericin in the pipette. The cells were depolarized to the potential eliciting maximum $\mathrm{Ca}^{2+}$ current for $20 \mathrm{~ms}$, in order to primarily recruit the readily-releasable pool of vesicles (RRP; Moser and Beutner, 2000; Beutner and Moser, 2001). By sequentially changing the extracellular $\left[\mathrm{Ca}^{2+}\right]$ (Figure 3.18b), the single channel current $\left(\mathrm{i}_{\mathrm{Ca}}\right)$ is altered, resulting in a difference in whole-cell $\mathrm{Ca}^{2+}$ influx and capacitance jump ( $\mathrm{I}_{\mathrm{Ca}}$ and $\Delta \mathrm{C}_{\mathrm{m}}$, Figure 3.18a-b). Alternatively, $\mathrm{Ca}^{2+}$ influx can be manipulated by slow perfusion of L-type $\mathrm{Ca}^{2+}$ channel blockers, in which case the number of open channels will be selectively reduced. 

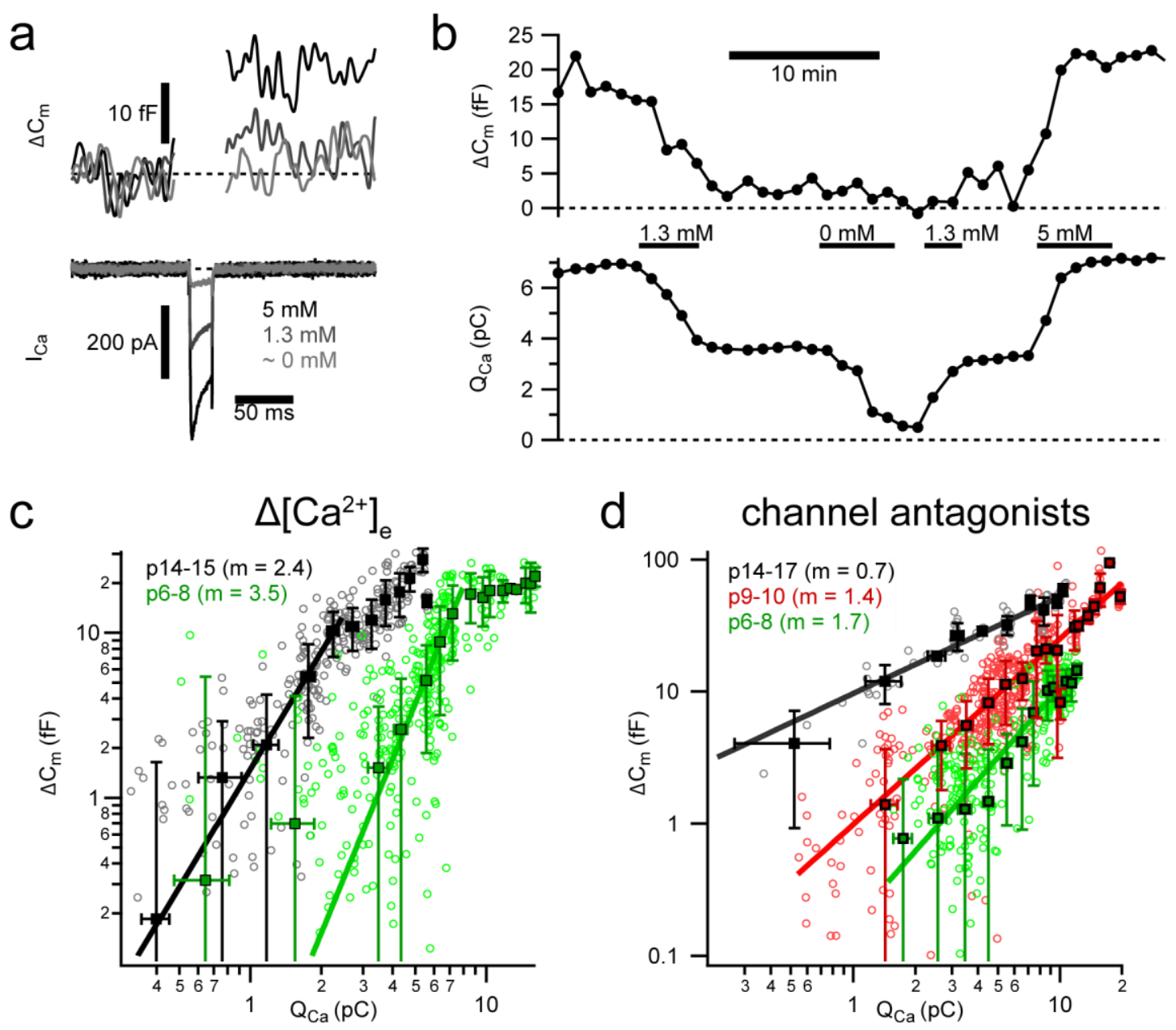

\section{Figure 3.18: Developmental change of apparent $\mathrm{Ca}^{2+}$ cooperativity in IHCs}

(a) Perforated-patch recording of a pre-hearing (p7) IHC. Depolarizations elicited inward $\mathrm{Ca}^{2+}$ current $\left(\mathrm{I}_{\mathrm{Ca}}\right.$, lower panel) and triggered change in membrane capacitance $\left(\Delta \mathrm{C}_{\mathrm{m}}\right.$, upper panel), a measure of vesicle fusion. Increasing extracellular $\left[\mathrm{Ca}^{2+}\right]\left(\left[\mathrm{Ca}^{2+}\right]_{\mathrm{e}}\right)$ increases both $\mathrm{I}_{\mathrm{Ca}}$ and $\Delta \mathrm{C}_{\mathrm{m}}$. (b) Time course for $\Delta \mathrm{C}_{\mathrm{m}}$ and integrated $\mathrm{Ca}^{2+}$ influx $\left(\mathrm{Q}_{\mathrm{Ca}}\right)$ of the same IHC in (a) for repetitive $20 \mathrm{~ms}$ depolarization to potential eliciting maximum $\mathrm{I}_{\mathrm{Ca}}$ at $60 \mathrm{~s}$ interval. Experiment started at $5 \mathrm{mM}\left[\mathrm{Ca}^{2+}\right]_{\mathrm{e}}$ and periods where bath solution was slowly perfused with a different $\left[\mathrm{Ca}^{2+}\right]_{\mathrm{e}}$ are marked by horizontal bars. (c) Plot of $\Delta \mathrm{C}_{\mathrm{m}}$ against $\mathrm{Q}_{\mathrm{Ca}}$, in both pre-hearing (p6-8, green, $\mathrm{n}=7 \mathrm{IHCs}$ ) and hearing (p14-15, grey, $\mathrm{n}=6 \mathrm{IHCs}$ ) IHCs when $\left[\mathrm{Ca}^{2+}\right]_{\mathrm{e}}$ was altered. Each small circle represents an individual response to depolarization, while squares represent the binned average of each age group. Solid lines represent best fit power functions with the form: $\Delta \mathrm{C}_{\mathrm{m}}=\mathrm{A}\left(\mathrm{Q}_{\mathrm{Ca}}\right)^{\mathrm{m}}$ to the low $\mathrm{Q}_{\mathrm{Ca}}$ portion of the data. The values of $m$ are shown in brackets. (d) Same kind of plot as in (c) but in these experiments $\mathrm{Ca}_{\mathrm{v}} 1.3$ channels were progressively blocked by slow perfusion of $10 \mu \mathrm{M}$ nifedipine or isradipine (p14-17, grey, $\mathrm{n}=4 \mathrm{IHCs}$; 9 -10, red, $\mathrm{n}=7$ IHCs; 6 , green, $\mathrm{n}=6 \mathrm{IHCs})$. 
Plotting the $\Delta \mathrm{C}_{\mathrm{m}}$ against the current integral $\left(\mathrm{Q}_{\mathrm{Ca}}\right)$ reveals the apparent $\mathrm{Ca}^{2+}$-dependence of release (Figure 3.18c-d). In case of a " $\mathrm{Ca}^{2+}$-microdomain" coupling (Borst and Sakmann, 1996), the relationship should reflect the supralinear intrinsic $\mathrm{Ca}^{2+}$ dependence (Figure 3.16), no matter how the $\mathrm{Ca}^{2+}$ influx was manipulated. In contrast, a " $\mathrm{Ca}^{2+}$-nanodomain" control (Bertram et al., 1996) the relationship will differ between the two manipulations (Augustine et al., 1991). In experiments where extracellular $\left[\mathrm{Ca}^{2+}\right]$ was manipulated, a supralinear relationship between release and $\mathrm{Ca}^{2+}$ influx was observed in both pre-hearing and mature IHCs (Figure 3.18c). When the number of channels are manipulated (Figure 3.18d), mature IHCs (p14-17) showed a rather linear relationship (pooled: $\mathrm{m}=0.7$; average: $\mathrm{m}=1.01 \pm 0.23$; $\mathrm{n}=4$ IHCs) but those of p6-8 IHCs remained slightly supralinear (pooled: $\mathrm{m}=1.7$, $\mathrm{n}=6$ IHCs; average: $\mathrm{m}=2.47 \pm 0.31, \mathrm{n}=4 \mathrm{IHCs}$ ). This indicates that during development, the IHC ribbon synapse progressively switches to a tighter "nanodomain"-like coupling.

\subsection{Characterization of vglut1-pHluorin (vGpH) Reporter in IHCs}

While whole-cell capacitance measurements gave us a good impression of how the IHC ribbon synapse operates on average, it lacks the resolution to distinguish individual AZs in a single IHC. In order to elucidate whether the intracellular heterogeneity of synaptic $\mathrm{Ca}^{2+}$ signals translates into differences in exocytosis among the active zones and therefore is relevant for expanding the dynamic range of sound encoding, a genetically-encoded fluorescent reporter of exocytosis was tested in IHC.

Using an adeno-associated virus (AAV) produced by the lab of Sebastian Kügler (construct prepared by Kirsten Reuter), Organs of Corti were transduced to express the fusion protein vglut1-pHluorin ( $\mathrm{vGpH}$ ) (through embryonic injection of otocysts by Kirsten Reuter and Christiane Senger-Freitag). The fusion construct was created by inserting pHluorin, a $\mathrm{pH}-$ sensitive variant of green fluorescent protein (GFP) (Miesenböck et al., 1998; Sankaranarayanan et al., 2000), into an intraluminal loop of the vesicular glutamate transporter vglut1 (Balaji and Ryan, 2007). In the following section I will characterize the feasibility of using vGpH as an optical measurement of exocytosis in transduced IHCs.

\subsubsection{Transduction efficiency, expression level and subcellular localization}

First, the efficiency of transduction was investigated. Figure 3.19 shows several acutely excised preparations under live fluorescent imaging, demonstrating the spectrum of different 
transduction efficiencies achieved. Even at resting condition, fluorescence of vGpHexpressing IHCs can be observed (Figure 3.19f-h). Transfection efficiency varied between one to a few cells in the whole apical half turn (>50 cells, e.g. Figure $3.19 \mathrm{~h}$ ), to more than $90 \%$ transfection. In some cases, OHCs were also transfected (Figure 3.19f, g).

Also notable is that within the same preparation, a variability of fluorescence intensity among IHCs was always observed (e.g. Figure 3.19f). Experiments with transient exposure of $50 \mathrm{mM}$ $\mathrm{NH}_{4} \mathrm{Cl}$, which alkalizes the intravesicular lumen and results in an increase in pHluorin fluorescence (Miesenböck et al., 1998), showed a similar percentage fluorescence increase ( 2-fold) for IHCs with different baseline intensity (Figure 3.23b). Together with results from correlation analysis by immunohistochemistry (Figure 3.20), the variability of fluorescence was likely caused by a difference in expression level, probably due to different copy number of the expression construct. 


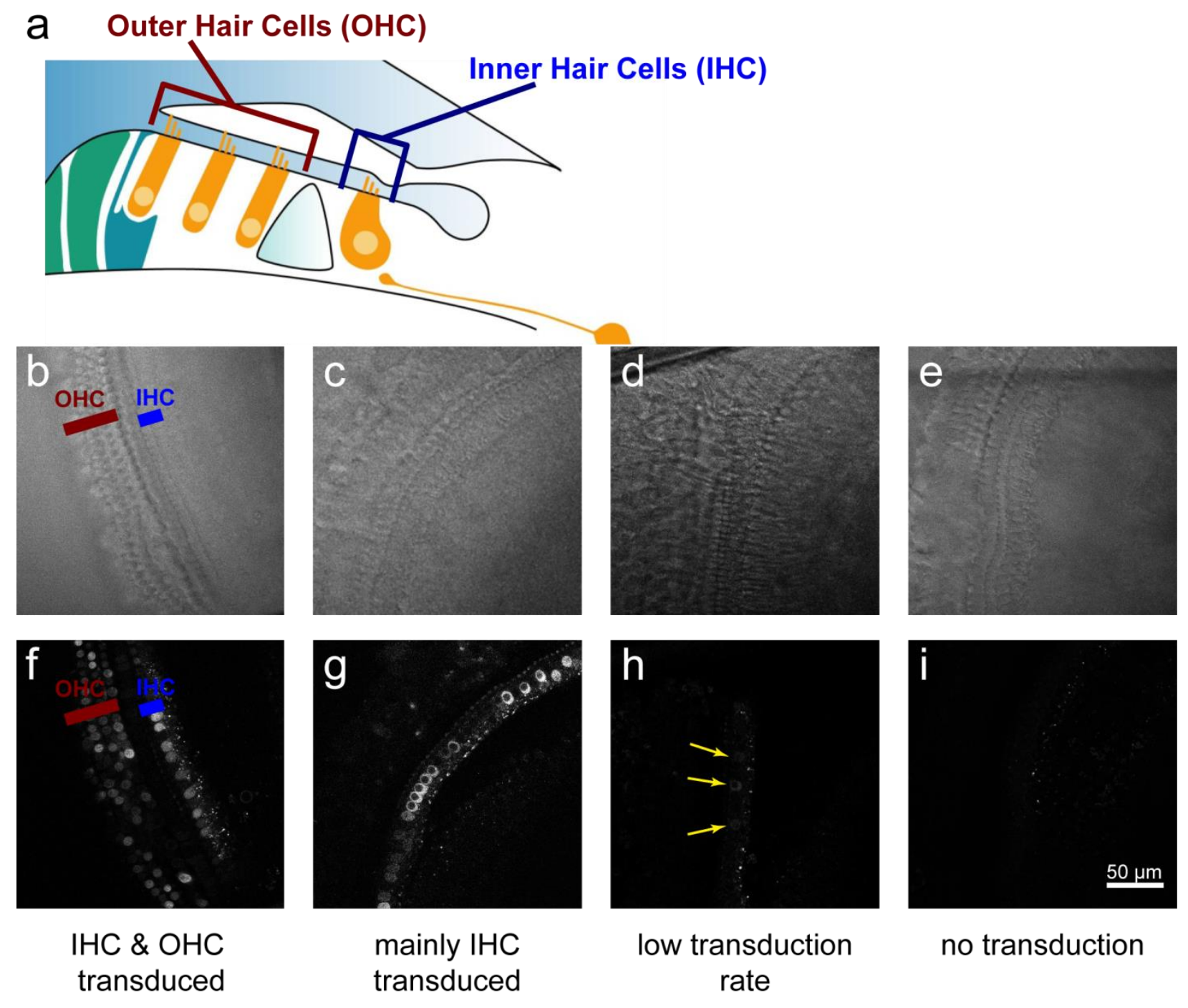

Figure 3.19: Spectrum of viral transduction in organ of Corti through embryonic injection

(a) Illustration of a cross-section of the organ of Corti, marking the positions of IHCs and OHCs. (b-e) Brightfield images of acutely dissected organs of Corti from mice injected with the vGpH virus. (f-i) Confocal section of the corresponding Organs in (b-e). Transfected IHCs identified in (h) are marked with yellow arrowheads.

To assess whether vGpH was correctly targeted to synaptic vesicle, transduced Organs of Corti were stained with an antibody against the endogenous IHC glutamate transporter, vesicular glutamate transporter 3 (vglut3) (Seal et al., 2008), and an antibody against transgenic vglut1 (Figure 3.20). The intensity of vglut1-staining reveals that expression levels are variable among transfected IHCs, as shown above in live imaging (Figure 3.19). In Figure 
3.20a, a correlation map of vglut1 and vglut3 immunofluorescence intensity is shown, in which the hue of each pixel was determined by the intensity ratio (see Methods). Region-ofinterest analysis at the basal end of the IHC yields an average correlation coefficient of $0.67 \pm 0.04$ ( $\mathrm{n}=8$ transduced IHCs, maximum intensity projection). It is apparent that each IHC seems to possess a specific hue, indicating a relative constant ratio between the two glutamate transporters within a given IHCs. Figure 3.20b exemplifies this finding by showing a scatter plot of pixel-wise comparison, where clusters of pixels with different slopes (and thus hue) appeared. Therefore, it can be concluded that the expression pattern of $\mathrm{vGpH}^{-}$ resembles that of endogenous vglut3 in transduced IHCs. On the other hand, the expression level of $\mathrm{vGpH}$ seems to have little impact on that of endogenous vglut3 (Figure 3.20c).

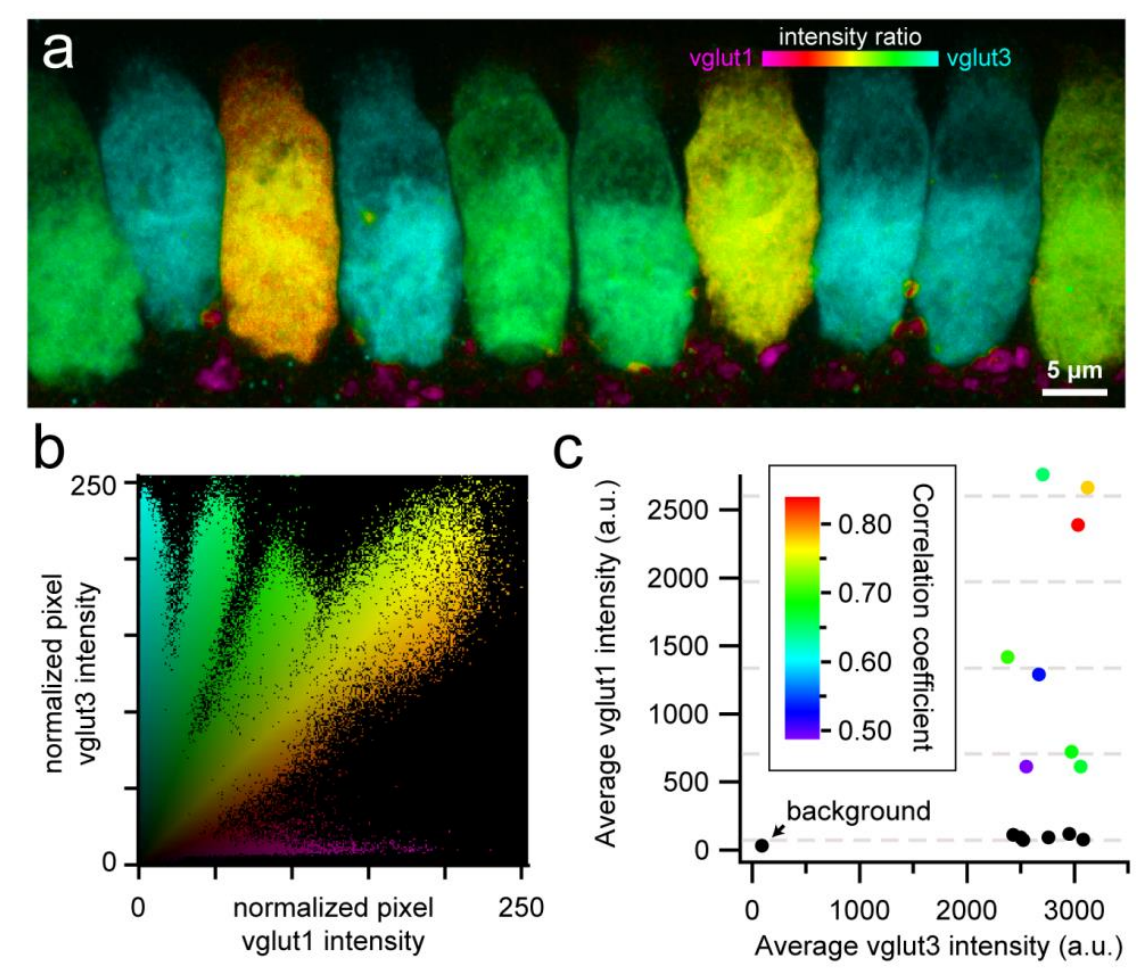

\section{Figure 3.20: Expression of $\mathrm{vGpH}$ does not affect expression, and resembles the expression pattern, of endogenous vglut3.}

(a) Immunohistochemistry labeling of vglut1 and vglut3 in a whole-mount organ of Corti, showing of a row of IHCs with different expression level of exogenous $\mathrm{vGpH}$. Lookup table was set to change hue based on ratio of the two labeling intensities in the maximum intensity projection: from magenta (vglut1 only) to yellow (equal portion) to cyan (vglut3 only). (b) Scatter plot of pixels in (a), with corresponding color lookup. (c) Average intensity of vglut1 against vglut3 in the basal part of 14 cells. Expression of $\mathrm{vGpH}$ does not affect that of vglut3. Expression level may be quantal, as approximated by dashed lines. 

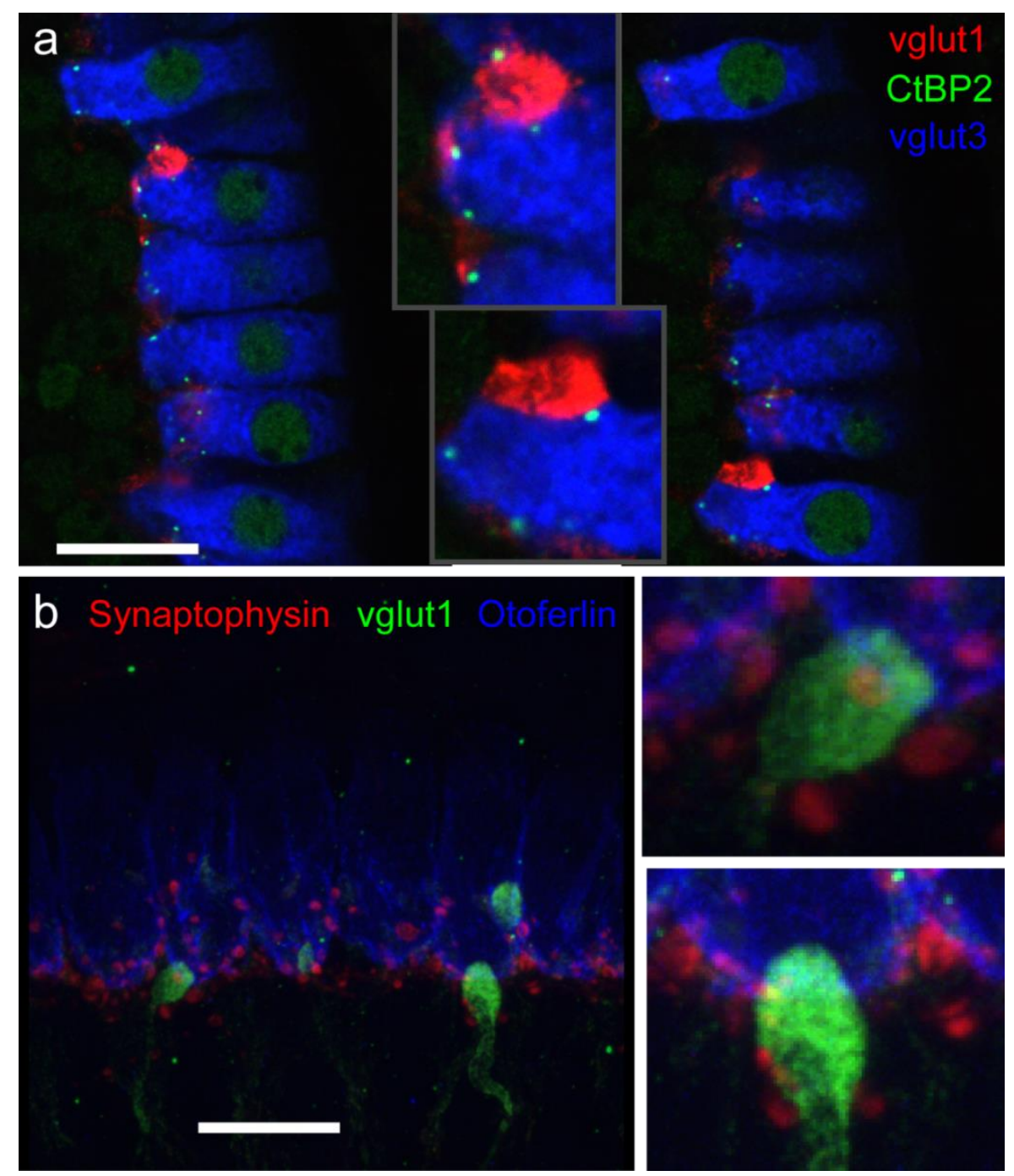

Figure 3.21: Bouton-like vglut1 immunoreactivity appears to be afferent terminals.

(a) Confocal projection of a transduced whole-mount organs of Corti stained with antibodies against vglut1 (red), CtBP2/RIBEYE (green) and vglut3 (blue).. Vglut1-positive boutons were observed juxtapose to synaptic ribbon revealed by CtBP2/RIBEYE staining. (b) Confocal projection of a transduced whole-mount organs of Corti stained for syanptophysin (red), vglut1 (green) and otoferlin (blue). Synaptophysin stained efferent terminals which did not localize but appeared to contact the vglut1positive boutons. Scale bars: $10 \mu \mathrm{m}$

Vglut1 staining was also sometimes observed in small bouton-like structure around the basal portion of IHCs. Based on their juxtaposition to synaptic ribbons (Figure 3.21a) and non-colocalization with synaptophysin (Figure 3.21b), these structures likely include afferent terminals of transfected SGN, or, maybe less likely, SGNs which endogenously express and target vglut1 to afferent terminals. Moreover, due to the variability of transfection, we also 
cannot exclude that some of the vglut1-positive (magenta) structures in Figure 3.20 could be efferent terminals, which have a similar morphology as shown by synatophysin staining in Figure 3.21b.

The seemingly ubiquitous presence of $\mathrm{vGpH}$ within transfected IHCs (Figure 3.20) prompted the question whether the expressed protein is localized in subcellular compartments other than synaptic vesicles. It was shown in rat (Siegel and Brownell, 1986; Schug et al., 2006) that the cis-Golgi network (CGN) concentrated in the apical part of IHC and apparently colocalized with another vesicular marker otoferlin at epifluorescence resolution. If the same is true in murine IHCs, enrichment in Golgi apparatus could possibly explain the apical expression of vglut1-pHluorin, where no afferent synapses are found.

Indeed, confocal images of a vGpH expressing IHC stained with GM130, a cis-Golgi marker, (Figure 3.22a, deconvolved) revealed the apical localization of CGN in IHC. However, with a closer inspection of the staining pattern (Figure 3.22b-d), it is apparent that $\mathrm{vGpH}$ is not enriched in CGN. Immunoreactivity of GM130 even appears to preferentially localize in region devoid of $\mathrm{vGpH}$. This is exemplified by the line profiles of fluorescence intensity (Figure 3.22e) 

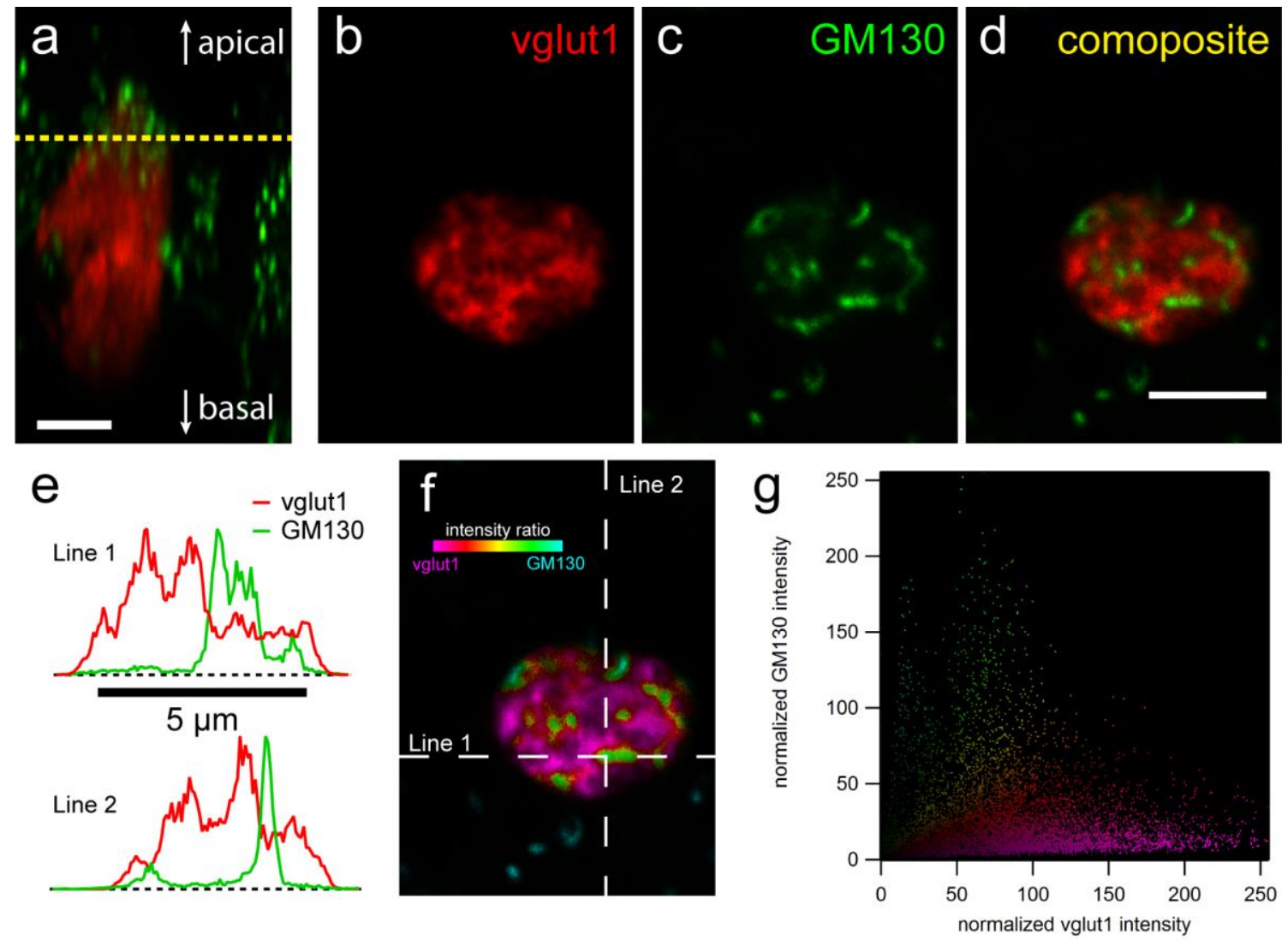

Figure 3.22: vGpH is not enriched in Golgi of transfected IHCs.

(a) Lateral projection of a confocal stack imaging a transduced IHC stained with anti-vglut1 (red) and anti-GM130 (green) antibodies. Images were deconvolved by 20 iterations of the Richardson-Lucy method. Scale bar: $4 \mu \mathrm{m}$. (b-d) Optical section at yellow dashed line in (a). (e)Line profiles or the intensities of vglut1 and Gm130 staining, positions shown in (f). (f) Correlation map between the two staining where hue changes from magenta (vglut1 only) to yellow (equal portion) to cyan (GM130 only).

(g) Scatter plot of the pixel intensities of the optical section with same lookup as (f).

\subsubsection{Measurement of intravesicular pH}

As another attempt to characterize the subcellular targeting of $\mathrm{vGpH}$, I tried to measure the luminal $\mathrm{pH}$ of the $\mathrm{vGpH}$-targetted compartment. Extracellular solutions of various $\mathrm{pH}$ containing $20 \mu \mathrm{M}$ carbonyl cyanide 4-(trifluoromethoxy)phenylhydrazone (FCCP), a protonophore and known mitochondrial blocker, were sequentially applied to a transduced organ of Corti (Figure 3.23a). FCCP blocks mitochondrial production of ATP by acting as a protonophore and depleting the proton gradient across the inner membrane. The same action should therefore also equilibrate the $\mathrm{pH}$ across cell membrane of intracellular organelles. By applying solutions with different $\mathrm{pH}$ and measuring the fluorescence of pHluorin molecules, 
we can estimate the resting $\mathrm{pH}$ of the compartment (Figure 3.23b-c). Each solution was applied for five minutes to allow equilibration and IHC nuclei were used as landmarks to correct for drifts in the vertical position. From the plots of Figure 3.23 the resting pH was estimated to be around $\mathrm{pH} 6.5$, and a brief application of $40 \mathrm{mM} \mathrm{NH}_{4} \mathrm{Cl}$ elevate the $\mathrm{pH}$ to around 7.5, leading to around 2-fold increase in fluorescence. This estimate is much higher than a previously measured pH of 5.5 in synaptic vesicle of neurons (Miesenböck et al., 1998)
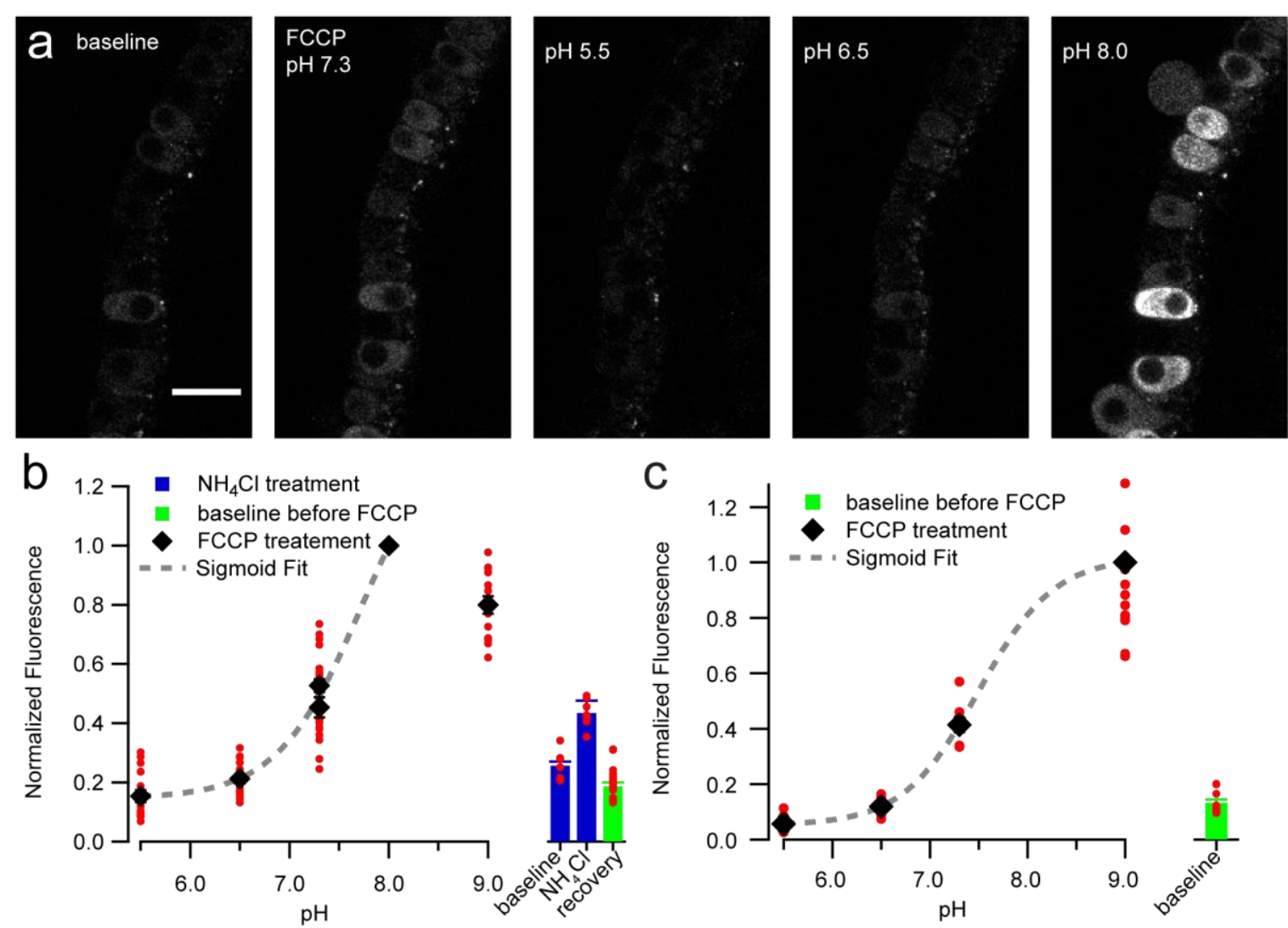

Figure 3.23: Calibrating pH of intracellular compartment by bath application of protonophore FCCP.

(a) Raw confocal images of transduced IHCs under perfusion of artificial perilymph (baseline) and solutions of different pH with $20 \mu \mathrm{M} \mathrm{FCCP.} \mathrm{(b)} \mathrm{Left} \mathrm{panel:} \mathrm{Average} \mathrm{normalized} \mathrm{fluorescence} \mathrm{intensity} \mathrm{of}$ IHCs against $\mathrm{pH}$ of FCCP solution. Right panel: Average intensity during $\mathrm{NH}_{4} \mathrm{Cl}$ treatment under the same normalization. Experiment was performed in the following order: Ringer, $\mathrm{NH}_{4} \mathrm{Cl}$, Ringer, $\mathrm{pH} 7.3$, pH5.5, pH6.5,pH7.3, pH8.0, pH9.0. Lower fluorescence intensity was observed at pH9.0 due to deterioration and swelling of cells, with possible increased bleaching at pH8.0. (c) Same experiment repeated starting from pH9.0. In both (b) and (c) the baseline fluorescence is similar to that at around pH6.5. Note the different normalization used in (b) and (c). 


\subsubsection{Depolarization evoked changes in fluorescence}

In order to assess whether $\mathrm{vGpH}$ in viral-transduced IHC can report synaptic vesicle fusion, I performed whole-cell patch-clamp recordings on these IHCs. Similar to $\mathrm{Ca}^{2+}$ imaging, a TAMRA-conjugated RIBEYE-binding peptide was introduced into the cell to visualize synaptic AZ. Figure 3.24 shows an example in which a step depolarization induced a stepwise change in membrane capacitance $\left(\Delta \mathrm{C}_{\mathrm{m}}\right)$ as well as an increase of pHluorin fluorescence $\left(\Delta \mathrm{F}_{\mathrm{vGpH}}\right)$ at the $\mathrm{AZ}$ (Figure 3.24c-d). In a number of trials (including the example in Figure 3.24), repetitive stimulation revealed hotspots of $\Delta \mathrm{F}_{\mathrm{vGpH}}$. In many cases, however, $\Delta \mathrm{F}_{\mathrm{vGpH}}$ signal diminishes after one or two repetition despite persistent increase in capacitance.

To access the effect of bleaching, the same imaging protocol (scan rate, image size, laser power) was performed on transduced IHCs exposed to $50 \mathrm{mM} \mathrm{NH}_{4} \mathrm{Cl}$. Figure 3.25a shows the average per frame fluorescence of the time series, fitted with a double exponential function:

$$
y=y_{0}+A_{1} \exp \left(-t / \tau_{1}\right)+A_{2} \exp \left(-t / \tau_{2}\right)
$$

In all traces, a rapid decline in fluorescence $\left(\tau_{1}=0.4 \mathrm{~s}\right)$ was observed, followed by a slower decline $\left(\tau_{2}=23.6 \mathrm{~s}\right)$. The rapid component represents a small fraction $\left(\mathrm{A}_{1}=0.11\right)$ of the fluorescence while the slower component represents around half $\left(\mathrm{A}_{2}=0.48\right)$. The presence of multiple components in have been reported in FRAP (fluorescence recovery after photobleaching) experiments for GFP and its variants, and the first rapid component was attributed to a reversible photoswitching process (Sinnecker et al., 2005; Mueller et al., 2012). The second component, on the other hand, represents the decline in fluorescence due to irreversible photobleaching. From the time constant $\tau_{2}$ and amplitude $A_{2}$, I calculated an initial rate of $0.02 \mathrm{~s}^{-1}$, translating into a $0.2 \%$ per frame bleaching rate $(10 \mathrm{~Hz}$ scanning rate). This is much higher than in another study using $\mathrm{vGpH}$ under epifluorescence, in which single vesicle resolution was achieved $(0.01 \%$ per frame at $5.9 \mathrm{~Hz}$, Balaji and Ryan, 2007). 

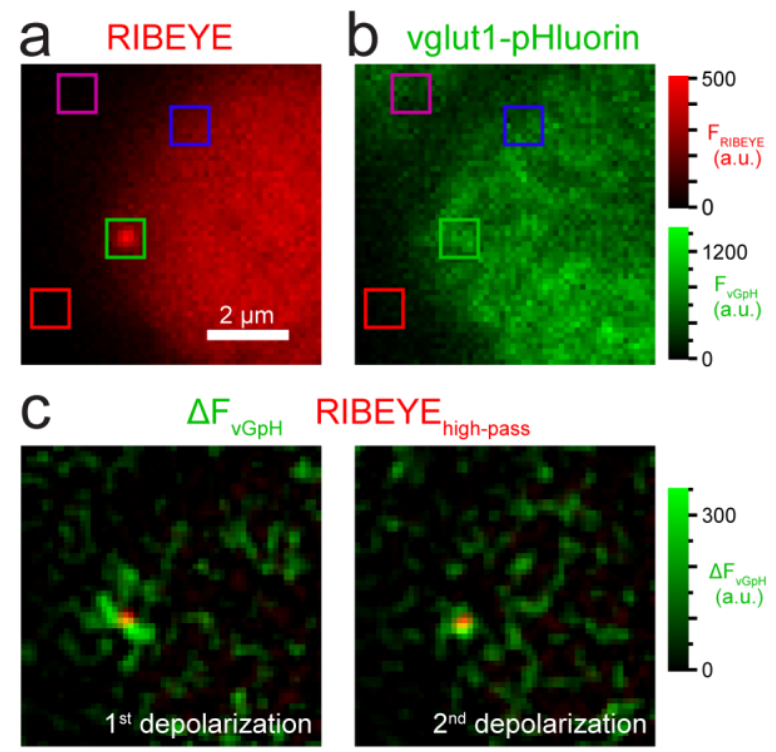

RIBEYE
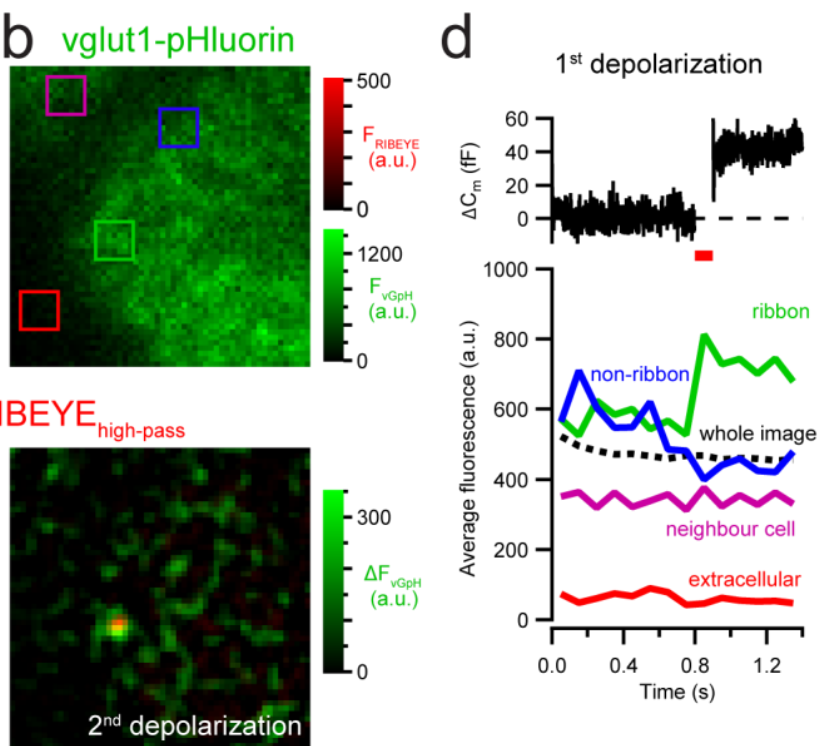

$2^{\text {nd }}$ depolarization

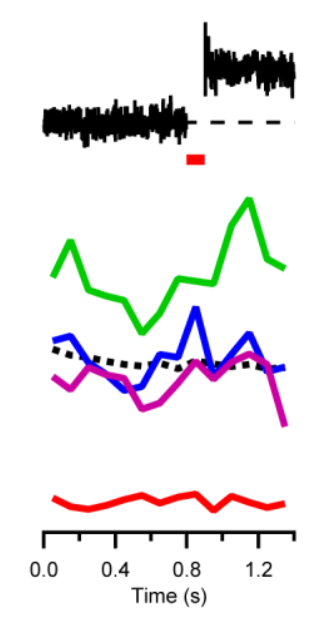

Figure 3.24: Depolarization of vGpH expressing IHCs reveals hotspots of vesicle fusion.

(a) Fluorescence of TAMRA-conjugated RIBEYE-binding peptide (monomer) in a vglut1-pHluroin expressing IHC under whole-cell patch-clamp configuration. A synaptic ribbon can be identified (green box). Colored boxes mark regions-of-interest (ROIs) analyzed in (d). (b) Simultaneously acquired fluorescence of $\mathrm{vGpH}$. Both (a) and (b) are averages of 15 frames acquired at $10 \mathrm{~Hz}$ during the entire stimulus protocol shown in (d). (c) Increase in $\mathrm{vGpH}$ fluorescence $\left(\Delta \mathrm{F}_{\mathrm{vGpH}}\right.$, green) after a $100 \mathrm{~ms}$ depolarization to $-17 \mathrm{mV}$, calculated as the difference between the 5-frame averages before and after the depolarization. The $\Delta \mathrm{F}_{\mathrm{vGpH}}$ channel were subjected to a $5 \mathrm{x} 5$ pixel Gaussian filter for display. RIBEYE channel (red) was subjected to a high pass filter to highlight position of ribbon (subtraction of image blurred by 3 passes of a $5 \times 5$ pixel Gaussian filter). Two repetitions show similar "hotspot" of $\Delta \mathrm{F}_{\mathrm{vGpH}}$ at the ribbon (d) Whole cell capacitance change $\left(\Delta \mathrm{C}_{\mathrm{m}}\right.$, top) and $\mathrm{vGpH}$ fluorescence at different ROIs (bottom) during the stimuli. Note the stepwise increase in fluorescence at the ribbon during the first depolarization. Fluorescence change is less obvious in the second depolarization, despite robust $\Delta \mathrm{C}_{\mathrm{m}}$ (first: $40.8 \mathrm{fF}$; second: $32.0 \mathrm{fF}$ ). For this experiment, $1 \%$ of maximum intensity was used for the $488 \mathrm{~nm}$ laser line. 

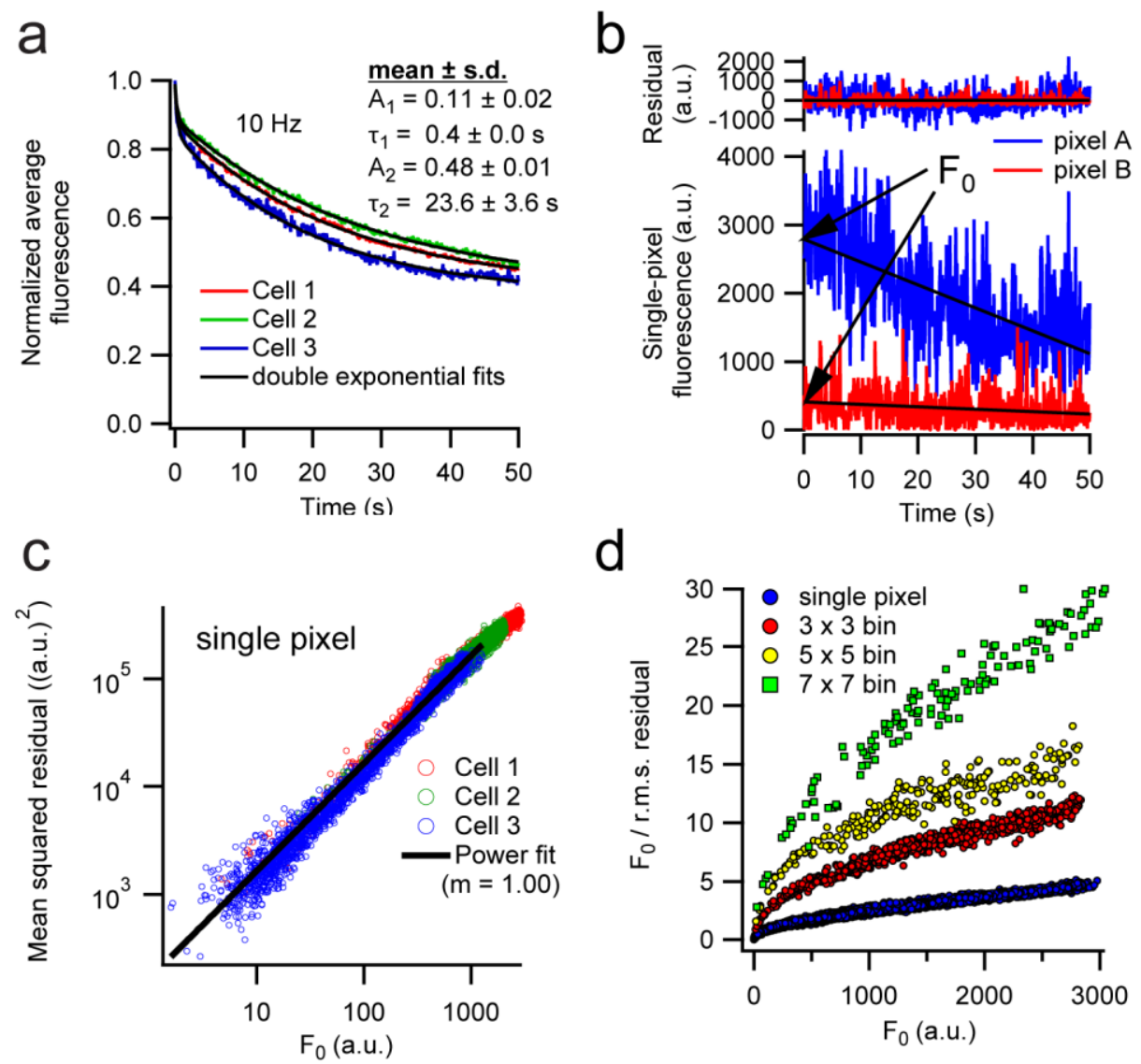

\section{Figure 3.25: Assessment of bleaching rate and signal-to-noise ratio of pHluorin imaging}

(a) Normalized average per frame fluorescence of 3 time series of $\mathrm{vGpH}$ expressing IHCs exposed to $50 \mathrm{mM} \mathrm{NH} \mathrm{NH}_{4} \mathrm{Cl}$, acquired at same settings as in Figure $3.24(10 \mathrm{~Hz}, 1 \%$ maximum laser intensity and $56 \times 56$ pixel frame). Bleaching was assessed by fitting a double exponential function to the traces. A small but rapid decline in fluorescence $\left(\tau_{1}=0.4 \mathrm{~s}\right)$ was observed in the beginning of the time series, followed by a slower persistent decline $\left(\tau_{2}=23.6 \mathrm{~s}\right)$. From $\tau_{2}$, an estimate of $0.2 \%$ per frame bleaching rate was obtained. (b) Fluorescence traces (bottom) of two pixels from cell 1. A line fit (black traces) was used to estimate the baseline fluorescence $\left(\mathrm{F}_{0}\right)$ and noise (residual, top). (c) Plotting the mean squared residual against $\mathrm{F}_{0}$ in logarithmic scale reveals a slope $(\mathrm{m})$ of one, an indication of Poisson noise. (d) Spatial binning improves Signal-to-noise ratio. The ratio of $\mathrm{F}_{0}$ to the root-mean-square (r.m.s.) of residual was plotted against $\mathrm{F}_{0}$. SNR of single pixel (blue symbols) was low even at high intensity. Analysis was repeated with binning of small squared ROIs (size indicated in legend). Exponential fits were used for the less noisy $7 \times 7$ bins, which was also the ROI size used in Figure 3.24.

The noise level of the imaging system was also assessed. As shown in Figure $3.25 \mathrm{~b}$, the fluorescence intensity was extracted for individual pixels. Each trace was then fitted with a 
line, which is more robust than an exponential function for low intensity traces. The baseline fluorescence $F_{0}$ was taken as the y-intercept of line fit. Noise can be estimated by the residual from the fit. Mean squared residuals for individual pixels are calculated and plotted against $\mathrm{F}_{0}$ in Figure 3.25c, which showed linear relationship (Power fit: $y=A F_{0}{ }^{m}, \mathrm{~m}=1.00$ ). This suggests the noise is dominated by the Poisson shot noise, instead of multiplicative Gaussian noise that could result during amplification. Signal-to-noise ratio (SNR), here approximated by dividing $\mathrm{F}_{0}$ by the root-mean-square (r.m.s.) of the fit residuals, was rather low for single pixels, which can be improved by binning Figure 3.25d. Here, $\mathrm{F}_{0}$ was defined as the "signal" because no stimulation was performed. This SNR estimate is therefore an overestimation because (1) the average fluorescence was lower than $\mathrm{F}_{0}$ due to bleaching; and (2) baseline fluorescence will contribute to the shot noise for a $\Delta \mathrm{F}$ signal. 


\section{Discussion}

\subsection{Release machinery of IHC ribbon synapse during postnatal development}

It has been reported that during postnatal development of mouse (Beutner and Moser, 2001; Johnson et al., 2005) and gerbil (Johnson et al., 2009) IHCs, the efficiency of $\mathrm{Ca}^{2+}$ influx in

triggering exocytosis increases. In addition, a linearization or reduction in apparent $\mathrm{Ca}^{2+}$ cooperativity of release was reported in these studies, depending on the species and tonotopic region. The lack of synaptotagmin IV (Syt4), myosin VI (myoVI) or mutation in the transmembrane cochlear-expressed gene 1 (tmc1) have been observed to abolish this linearization (Marcotti et al., 2006; Roux et al., 2009; Johnson et al., 2010). However, IHCs of adult tmc1 and myoVI mutants exhibit other immature phenotypes, hinting at a gross developmental defect (Marcotti et al., 2006; Roux et al., 2009). From data on the Syt4 mutant, Johnson and colleagues (2010) proposed a developmental switch of molecular machinery, but no direct evidence on difference in $\mathrm{Ca}^{2+}$ dependence of release have been demonstrated between IHCs before and after hearing onset. In addition, otoferlin, a putative $\mathrm{Ca}^{2+}$ sensor in IHCs, is required for exocytosis in IHCs from $4^{\text {th }}$ postnatal day (Beurg et al., 2010), way before the onset of hearing. In order to probe whether a postnatal change in $\mathrm{Ca}^{2+}$ sensor properties occurs, we preformed $\mathrm{Ca}^{2+}$-uncaging in p6-8 and p14-17 IHCs (see section 3.2.1)

\subsubsection{Developmental changes of in flash responses}

The observed smaller amplitude in p6-8 IHCs (Figure 3.15) can be partly explained by the lower number of available vesicles. A p14 ribbon observed in electron microscopy occupied on average four ultrathin sections $(70 \mathrm{~nm})$, while a p6 ribbon typically spanned 1.5 sections. Taking into consideration the halving of the ribbon number from $\mathrm{p} 6$ to $\mathrm{p} 14$ (see section 3.1.4) and data in Table 2, it can be estimated that a two-fold developmental increase in the total number of ribbon-tethered vesicles per IHC occurs during this period ( 210 in p6 vs $\sim 410$ in p14). Nevertheless, the reason behind the huge discrepancy between the size of RRP (10$20 \mathrm{fF}$, Moser and Beutner, 2000; Beutner and Moser, 2001; Johnson et al., 2005) and the size of the flash responses (p6: $324 \mathrm{fF}$; p14-17: $1161 \mathrm{fF}$; and p14-25: $1520 \mathrm{fF}$ in Beutner et al., 2001) has remained elusive. Using a conversion factor of $45 \mathrm{aF}$ (mature) and $48 \mathrm{aF}$ (immature) per vesicle (Neef et al., 2007), the estimated numbers of ribbon-associated vesicle match 
better with RRP (220-440 vesicles) than the flash response (6750 or 25800-33800 vesicles). The flash responses, therefore, likely include replenishing vesicles. In addition, the possibility of $\mathrm{Ca}^{2+}$ dependent extrasynaptic exocytosis cannot be excluded.

The $\mathrm{C}_{\mathrm{m}}$ increase in most mature (31 of 32; p14-17) IHCs could be approximated by a double exponential function, while responses in almost half (18 of 40) of the prehearing (p6-8) IHCs exhibited only a single component. We observed that the rate constants of responses with only one exponential component were comparable to that of the second (slower) component of two-component responses. Interestingly, similar observations were reported earlier in bovine chromaffin cells (Heinemann et al., 1994). Heinemann and colleagues (1994) postulated two hypotheses. First, the two capacitance time courses may correspond to two different cell types. Alternatively, the two components may correspond to parallel pathways of secretion, and some cells only have the slower pathway while others have both. Considering the first hypothesis, a mixture of cell type is improbable in our case as the response in mature IHCs does not such segregation. The existence two populations could also be due to variability in a developmental switch of release mechanism. Although both types of responses were observed in p6, p7 and p8 (single/total: 1/6, 14/27, 3/7, respectively), a large variability in maturational time course among IHCs can mediate such observation. In addition, further supporting this hypothesis is the observed increase in contribution of the fast component (p6-8: 31\%; p14-18: 49\%; p14-25: 70\% in Beutner et al., 2001) for cells showing two components. It would be interesting to see whether more two-component IHCs would be observed in intermediate age (e.g. p10) than in p6-8.

Another possibility, corresponding to the second hypothesis, is that some of the cells have already depleted their fast component due to the relatively high pre-flash $\left[\mathrm{Ca}^{2+}\right]$ in the current dataset $\left(\left[\mathrm{Ca}^{2+}\right]_{\text {pre }} ; \mathrm{p6}-8: 5.2 \pm 0.5 \mu \mathrm{M}, \mathrm{n}=40 ; \mathrm{p} 14-17: 4.9 \pm 1.0 \mu \mathrm{M}, \mathrm{n}=16\right)$. Although the use of the low affinity indicator mag-fura- $2\left(K_{d}=35.1 \mu \mathrm{M}\right.$, Naraghi, 1997) did not allow accurate measurement of baseline $\left[\mathrm{Ca}^{2+}\right]$, a qualitative inspection reveals that IHCs with a single component in their flash responses actually has a lower average $\left[\mathrm{Ca}^{2+}\right]_{\text {pre }}(3.8 \pm 0.4 \mu \mathrm{M})$ than that of their double exponent counterparts $(6.3 \pm 0.8 \mu \mathrm{M})$. Response amplitude did not seem to correlate with $\left[\mathrm{Ca}^{2+}\right]_{\text {pre }}(\mathrm{r}=-0.06, \mathrm{p}=0.71$; Figure $4.1 \mathrm{~b})$. This argues against the depletion of fast component by high baseline $\left[\mathrm{Ca}^{2+}\right]$. On the contrary, the higher $\left[\mathrm{Ca}^{2+}\right]_{\text {pre }}$ may seem to 
facilitate and speed up release, creating a faster first component in two-component cells. This hypothesis is in agreement with the facilitation observed with pre-depolarization in pairrecordings (Goutman and Glowatzki, 2011). Nevertheless, it remains speculative and need to be further tested with experiments.
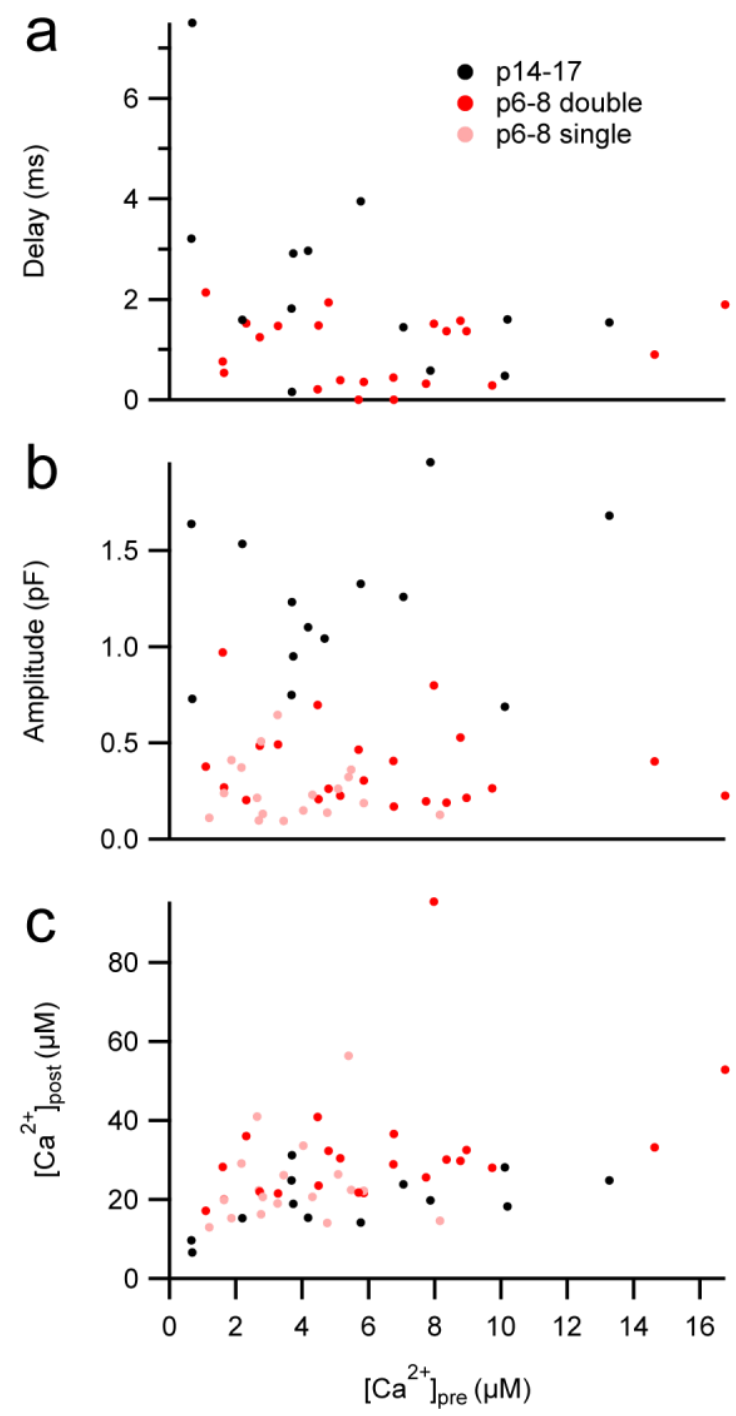

Figure 4.1: Properties of $\mathrm{Ca}^{2+}$ uncaging responses versus pre-flash $\left[\mathrm{Ca}^{2+}\right]_{\mathrm{i}}$

(a) Response delay (Pearson's correlation, $r=-0.15, \mathrm{p}=0.37)$, (b) amplitude $(\mathrm{r}=-0.06, \mathrm{p}=0.71$ ), and (c) post-flash $\left[\mathrm{Ca}^{2+}\right](\mathrm{r}=0.40, \mathrm{p}=0.01)$ plotted as a function of $\left[\mathrm{Ca}^{2+}\right]_{\text {pre }}$. Black circles represent responses from subset of mature (p14-17) IHCs where $\left[\mathrm{Ca}^{2+}\right]_{\text {pre }}$ data was available. Red and pink circles represents responses from pre-hearing (p6-8) IHCs which has a double (red) or single (pink) exponential time course. Note that the low $\left[\mathrm{Ca}^{2+}\right]_{\text {pre }}$ cannot be accurately determined with mag-fura- $2\left(\mathrm{~K}_{\mathrm{d}}=35.1 \mu \mathrm{M}\right)$. 
Although the limited range of postflash $\left[\mathrm{Ca}^{2+}\right]$ in experiments presented here was not sufficient for the derivation of a kinetic model, rate constants and delay of the responses in pre-hearing (p6-8) and mature (p14-17) IHCs were largely similar (Figure 3.16). This provides evidence that release of vesicles in IHCs is likely mediated by the same $\mathrm{Ca}^{2+}$ sensor in both two age groups. Similar experiments on the calyx of Held synapse have shown a slight developmental shift in $\mathrm{Ca}^{2+}$ sensitivity in mice (p9-11 vs p16-19, Wang et al., 2008) but not in rats (p8-9 vs p12-15, Kochubey et al., 2009).

\subsection{2 $\mathrm{Ca}^{2+}$ dependence of release in IHC and molecular candidates for $\mathrm{Ca}^{2+}$ sensor}

As described before (Beutner et al., 2001), the release of vesicles in the IHC exhibits cooperativity for $\mathrm{Ca}^{2+}$ binding, showing a slope of 4-5 in the lower $\left[\mathrm{Ca}^{2+}\right]$ region measured (re-plotted in Figure 3.17). In the higher $\left[\mathrm{Ca}^{2+}\right]$ region, the release rate levels off and was proposed to be limited by the maximum release rate $\gamma$, which represent the intrinsic velocity of the fusion reaction.. In the new dataset (black circles in Figure 3.16), the release rate seemingly continued to increase with a steep slope at around $30 \mu \mathrm{M}\left[\mathrm{Ca}^{2+}\right]$ for both prehearing and mature IHCs. In addition, the delay to exocytic response was also shorter in the new dataset than reported by Beutner and colleagues (2001). Whether this is due to the utilization of a shorter laser pulse for uncaging instead of the temporally spreadout illumination ( $\sim \mathrm{ms}$ pulse, hardware specification by Rapp OptoElectronic) arc lamp, or the relatively elevated pre-flash $\left[\mathrm{Ca}^{2+}\right]$ is yet to be determined. The $2 \mathrm{kHz}$ capacitance sampling rate (see Material and Methods and Beutner et al., 2001) also limited the experimentally deduced rate constant. Future experiments should address the possibility of higher release rate constants by using sine waves of higher frequencies for capacitance measurements. Even more ideal, in terms of temporal resolution, would be the establishment of $\mathrm{Ca}^{2+}$ uncaging experiment using paired pre- and postsynaptic recordings (Goutman and Glowatzki, 2007), similar to those routinely performed in the calyx of Held synapse (e.g. Bollmann et al., 2000; Schneggenburger and Neher, 2000; Lou et al., 2005; Sun et al., 2007).

The exact identity of the $\mathrm{Ca}^{2+}$ sensor in IHC is, however, under discussion (Roux et al., 2006; Beurg et al., 2010; Johnson et al., 2010; Pangršič et al., 2010). Synaptotagmin IV (Syt4) was proposed to be the sensor responsible for linearization of $\mathrm{Ca}^{2+}$-dependence (Johnson et al., 2010), due to the inability of its $\mathrm{C}_{2} \mathrm{~A}$ domain to bind $\mathrm{Ca}^{2+}$ (Südhof, 2001). However, this 
hypothesis failed to explain the high cooperativity of intrinsic $\mathrm{Ca}^{2+}$-dependence (Beutner et al., 2001). Moreover, Syt4 was found to localize specifically with BDNF(brain-derived neurotrophic factor)-containing but not synaptophysin-marked vesicles in hippocampal neurons (Dean et al., 2009), and thus is believed to be involved in modulation rather than direct synaptic transmission. Otoferlin, a transmembrane protein containing multiple $\mathrm{C}_{2}$ domains, is another candidate $\mathrm{Ca}^{2+}$ sensor in IHC. $\mathrm{C}_{2}$-domains of otoferlin appeared to promote SNARE-mediated fusion in a reconstitutive system (Johnson and Chapman, 2010; but note the independence of release in IHC from neuronal SNAREs: Nouvian et al., 2011). In knockout mice of otoferlin, exocytosis in IHCs was abolished after postnatal day 4 (Beurg et al., 2010). A study on an additional otoferlin mouse mutant suggested additional roles of otoferlin in replenishment and possibly priming of synaptic vesicle (Pangršič et al., 2010). The multifunctionality of otoferlin is not unlikely, given its multi-domain structure and the many binding partners (for a review on otoferlin, see Pangršič et al., 2012). However, one should be cautious in pinpointing the $\mathrm{IHC} \mathrm{Ca}^{2+}$ sensor of release and be open to other potential candidates.

\subsection{From microdomain to nanodomain control of vesicle fusion}

Through a combination of immunohistochemistry and $\mathrm{Ca}^{2+}$ imaging, I demonstrated that $\mathrm{Ca}^{2+}$ channels and $\mathrm{Ca}^{2+}$ influx are progressive confined to $\mathrm{AZ}$ during postnatal development of IHC (Figure 3.8). Moreover, although whole-cell $\mathrm{Ca}^{2+}$ current declines during the second postnatal week (Figure 3.7; Beutner and Moser, 2001; Marcotti et al., 2003; Johnson et al., 2005), total synaptic $\mathrm{Ca}^{2+}$ influx did not increase (section 3.1.4). The apparent developmental increase in efficiency of whole-cell $\mathrm{Ca}^{2+}$ current in triggering exocytosis (Beutner and Moser, 2001; Johnson et al., 2005) can therefore be due to the reduction of extrasynaptic channels, which are not contributing to fast $\mathrm{Ca}^{2+}$-triggered exocytosis. The $\mathrm{Ca}^{2+}$ influx from extrasynaptic channels, however, may be involved during sustained release which could be inhibited by high concentration of EGTA (Moser and Beutner, 2000) and has similar dependence on cumulative $\mathrm{Ca}^{2+}$ influx in prehearing and hearing IHCs (Beutner and Moser, 2001).

\subsubsection{Developmental decrease in apparent $\mathrm{Ca}^{2+}$ cooperativity of release}

Earlier experiments have also probed at the apparent cooperativity of release in developing mouse IHCs (Johnson et al., 2005), and found a developmental linearization of release-influx 
relationship. However, the use of long depolarization $(100 \mathrm{~ms})$ and rupture-patch recording confounded the interpretation of results. Release measured after long depolarizations involve replenishment of the RRP, which may have a distinct $\mathrm{Ca}^{2+}$-dependence (Moser and Beutner, 2000). The use of a pre-defined amount of exogenous $\mathrm{Ca}^{2+}$ buffer is needed for rupture patch experiments, and may not represent the endogenous buffering situation especially across different age groups (Hackney et al., 2005). In this study, I performed experiments under perforated patch configuration to retain endogenous buffering, and restricted to short $20 \mathrm{~ms}$ depolarization, which selectively invokes RRP even at high extracellular $\left[\mathrm{Ca}^{2+}\right](10 \mathrm{mM}$, Moser and Beutner, 2000). In addition, $\mathrm{Ca}^{2+}$ influx was manipulated in ways that selectively changes the single channel current (through change of extracellular $\left[\mathrm{Ca}^{2+}\right]$ ) and channel open probability (through perfusion of L-type $\mathrm{Ca}^{2+}$ channel antagonist isradipine or nifedipine). This approach also allowed one to distinguish between a $\mathrm{Ca}^{2+}$ microdomain and a $\mathrm{Ca}^{2+}$ nanodomain control of vesicle fusion (see Figure 4.2 and following).

As shown in Results section, manipulation of single channel current, and thus the $\left[\mathrm{Ca}^{2+}\right]$ of the domain at the site of $\mathrm{Ca}^{2+}$ influx, reveals a supralinear relationship between release and $\mathrm{Ca}^{2+}$ influx in both pre-hearing and mature IHCs (Figure 3.18c). This was expected for both the $\mathrm{Ca}^{2+}$ microdomain and $\mathrm{Ca}^{2+}$ nanodomain models (Figure 4.2a, b, middle panels), since the slope in the logarithmic plot should reflect the supralinear intrinsic $\mathrm{Ca}^{2+}$ dependence of release (Figure 3.16 and Beutner et al., 2001). When the open probability of channels was manipulated through slow perfusion of antagonists, a change from supralinear relationship to linear relationship was observed (Figure 3.18d). Together, these results suggest in vesicle release in prehearing IHCs require concerted opening of multiple channels $\left(\mathrm{Ca}^{2+}\right.$ microdomain control), and mature into a tighter " $\mathrm{Ca}^{2+}$ nanodomain" control after hearing onset. Supporting the idea of tighter coupling, it was shown that rapid exocytosis in prehearing IHCs were more sensitive to $\mathrm{Ca}^{2+}$ buffering than mature IHCs (ratio of $\Delta \mathrm{C}_{\mathrm{m}}$ under endogenous or $5 \mathrm{mM}$ EGTA buffering, 10 ms depolarization: p6, 0.63; p14-25, 0.90; 20 ms: p6, 0.68; p14-25, 0.73; from Beutner and Moser, 2001). 


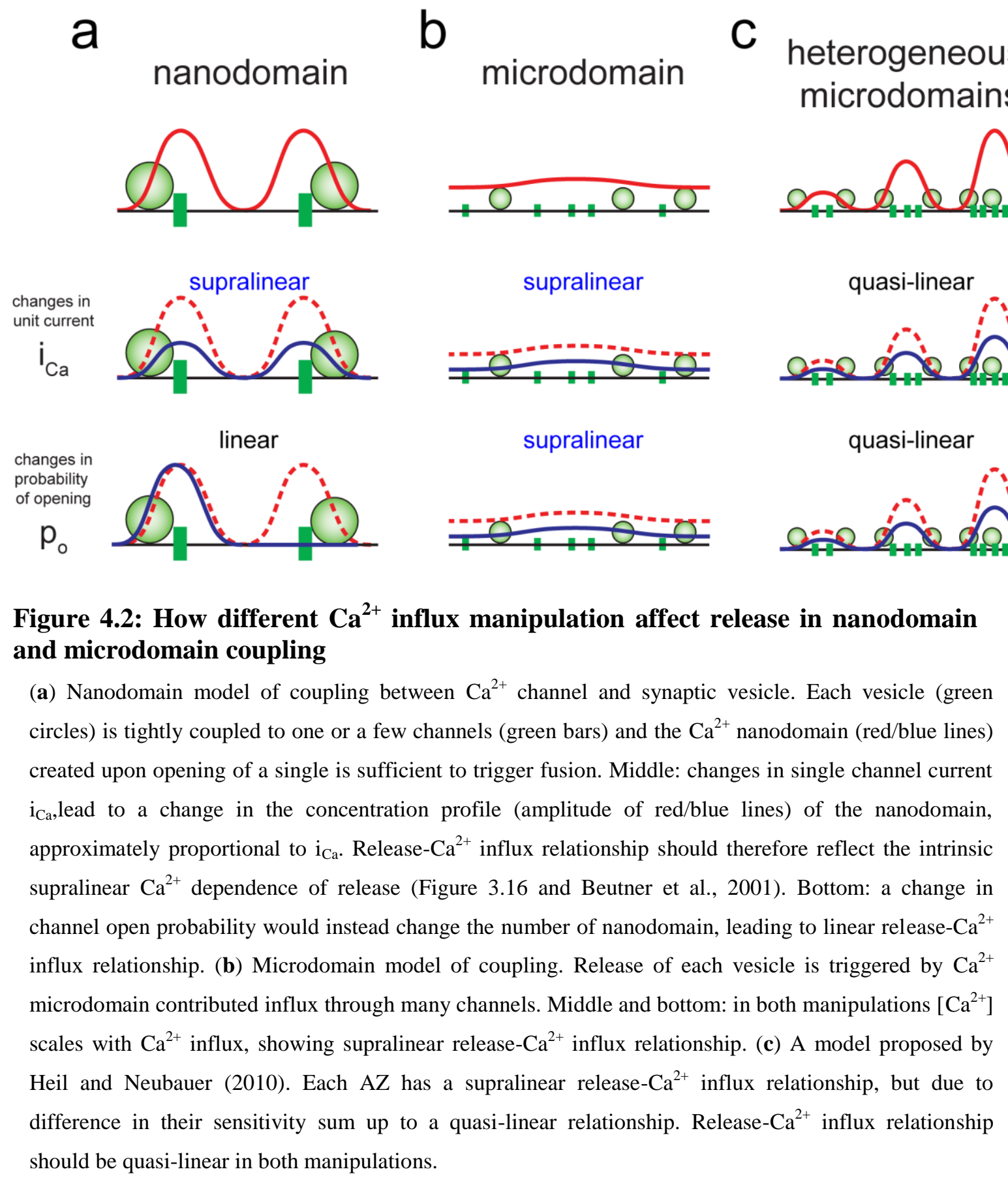

An interesting alternative hypothesis has been proposed by Heil and Neubauer (2010). They suggested that the quasi-linearity of hair cell release may also be achieved, by summing up release at AZs with different sensitivity, each of which is supralinear on its own. This idea becomes even more appealing with the observed developmental increase in heterogeneity of synaptic $\mathrm{Ca}^{2+}$ signal (see section 3.1.4 and Figure 3.10). However, if we assume that the 
release linearity in mature IHCs was due to the summation of many AZs under strict microdomain (supralinear) control, it should be preserved provided that $\mathrm{Ca}^{2+}$ influx at all $\mathrm{AZs}$ is similarly affected (Figure 4.2c). This was not the case when $\left[\mathrm{Ca}^{2+}\right]_{\mathrm{e}}$ was changed (Figure 3.18c and Brandt et al., 2005), where a supralinear relationship was instead observed. We cannot rule out the contribution of AZ heterogeneity, as a small decrease in cooperativity was observed in manipulation of $\mathrm{i}_{\mathrm{Ca}}$ (Figure 3.18c). It, however, cannot be the sole mechanism mediating the linearization of synaptic transfer function.

\subsubsection{Possible mechanisms for switch from microdomain to nanodomain}

Similar phenomenon of switching into tighter, nanodomain-like release coupling was observed in the Calyx of Held in mice (Fedchyshyn and Wang, 2005) and rat (Kochubey et al., 2009). In addition, it was later found in calyx of Held that a knockout or acute inhibition of septin 5 conferred mature apparent cooperativity in immature calyces (Yang et al., 2010). The protein was thought to control spatial coupling by preventing the close association of synaptic vesicles to AZs, and thus $\mathrm{Ca}^{2+}$ channels therein (Yang et al., 2010). This also argues for a biologically controlled coupling during development, rather than an "underdeveloped" synapse in the prehearing animal. However, whether this is applicable to the IHCs is yet to be determined.

Another factor that may contribute to changes in release coupling is the developmental shift in endogenous $\mathrm{Ca}^{2+}$ buffer concentrations. Less buffer creates a longer length constant for the $\mathrm{Ca}^{2+}$ domain around each $\mathrm{Ca}^{2+}$ channel (Naraghi and Neher, 1997), allowing more "domainoverlap" and thus more $\mathrm{Ca}^{2+}$ channel that can influence release of a single vesicle (Matveev et al., 2011). However, a study in rat organ of Corti using immunogold-labeling and electron microscopy have estimated a developmental decline of $\mathrm{Ca}^{2+}$ buffering proteins in IHCs (Hackney et al., 2005), opposite to what we would expect. On the other hand, Hackney and colleagues (2005), showed that this decline was mainly realized by the disappearance of the fast, high-affinity buffer calbindin ( $\mathrm{Kd}=393 \mathrm{nM}$, Faas et al., 2011). How and whether a switch in buffer type can contribute to the release coupling will be an interesting question awaiting further investigation. 


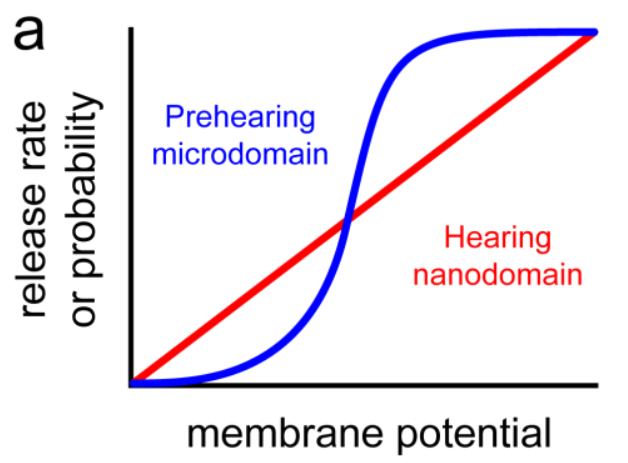

b

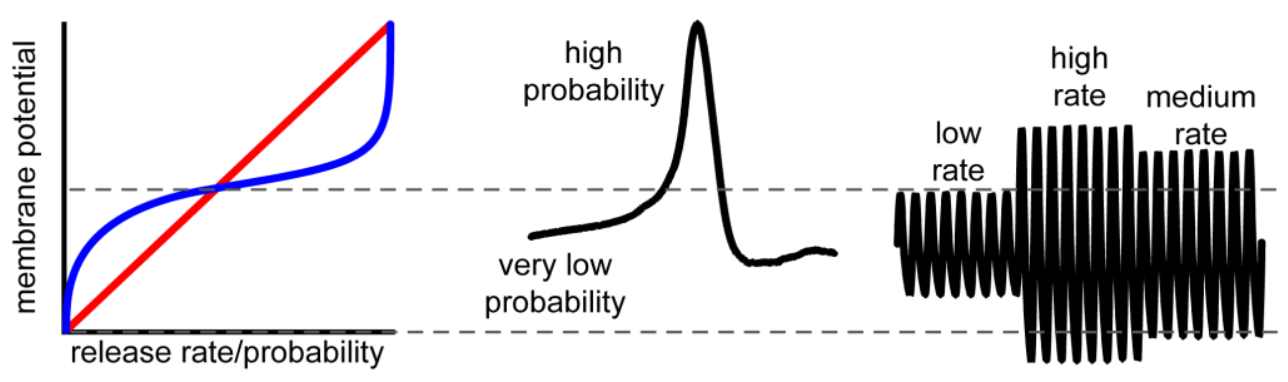

Figure 4.3: Proposed biological relevance in linearization of release cooperativity in IHC

(a) Schematic showing relationship between release rate (or approximately equivalent, release probability) and membrane potential in a supralinear prehearing IHC (blue line) and a linear mature IHC (red). Saturation or pool depletion lead to a sigmoidal shape in the supralinear case. For simplicity, non-linearity of $\mathrm{Ca}^{2+}$ channel activation was not considered here. (b) Relating the relationship in (a) to natural electrical activity of prehearing and mature IHCs. High cooperativity in prehearing IHC ensures release only at peak of an AP, while linear cooperativity in hearing IHC provide a graded release rate suitable for encoding a wide dynamic range of sound intensities.

Biologically, the developmental shift from a supralinear to linear "synaptic transfer function" (release vs $\mathrm{Ca}^{2+}$ influx) in IHCs may complement their functional switch. During early postnatal period, transmitter release in IHCs is evoked by $\mathrm{Ca}^{2+} \mathrm{APs}$, which leads to bursting of postsynaptic SGNs (Tritsch et al., 2010). The relatively depolarized resting membrane potential (around -60 to $-55 \mathrm{mV}$, Figure 3.1 and Johnson et al., 2012) in the prehearing IHC would lead to the activation of $\mathrm{Ca}^{2+}$ channel, and a high cooperativity would ensure that release only happens during a $\mathrm{Ca}^{2+} \mathrm{AP}$ when the membrane is strongly depolarized (Figure $4.3 \mathrm{~b})$. On the other hand, the linear synaptic transfer function allows the mature IHC to create different release rate based on the strength of stimulation. This would increase the dynamic range for which the IHC can operate, as suggested for hair cell and other sensory ribbon-type 
synapses which sustained release under tonic stimuli (Witkovsky et al., 1997; Moser and Beutner, 2000; Thoreson et al., 2003; Brandt et al., 2005; Johnson et al., 2005; Jarsky et al., 2010).

\subsection{Refinement of synaptic scaffold and postsynaptic density during development}

During postnatal development, the synapse between an IHC and a SGN afferent terminal originally form multiple AZs and postsynaptic densities (PSD), and later mature into a single AZ/PSD pair (Figure 3.13 and Figure 3.14). The precise mechanisms mediating the transition into a single synaptic contact remain to be investigated. However, this process seems to be independent of presynaptic exocytosis, as discrete glutamate receptor clusters were still observed in p15 otoferlin knockout mice, which lacks depolarization-triggered exocytosis (Roux et al., 2006). The pore forming $\alpha 1$ subunit of Cav1.3 channels also seems not to be directly involved, as the formation of ribbon-GluA pair was normal in the knockout animal, until degeneration occurs between p30-55 (Nemzou et al., 2006).

There are at least two non-exclusive hypotheses: 1) merging of immature contacts and 2) pruning of all but one of the immature contacts. Merging may be mediated by interactions of scaffolds at the presynaptic and/or postsynaptic level and possibly involves trans-synaptic regulation. Such cluster-cluster interaction may join multiple small ribbons, presynaptic $\mathrm{Ca}^{2+}$ channel clusters and postsynaptic GluA clusters into larger singular structures. Such a mechanism seems plausible for maturation of the large complex rings of GluA clusters (Figure 3.13a) to small ring-like GluA clusters (Meyer et al., 2009). Moreover, strong RIBEYE-RIBEYE interactions observed previously (Magupalli et al., 2008) and reports on structural assembly of retinal ribbon during synaptogenesis (Regus-Leidig et al., 2009) suggested the possibility of a yet-to-be-demonstrated fusion between AZ-anchored ribbons. Finally, AZ merging could readily explain the gain of large $\mathrm{AZ}$ with intense $\mathrm{Ca}^{2+}$ signals.

Pruning is a well-established mechanism for refining synaptic connections in general (Goda and Davis, 2003) and also a popular hypothesis for the maturation of IHC afferent connectivity (Sobkowicz et al., 1982; Nemzou et al., 2006; Huang et al., 2007; Sendin et al., 2007; Defourny et al., 2011; Bulankina and Moser, 2012). The presence of ribbons may serve to stabilize the contacts, such that ribbonless contacts will be preferentially pruned. However, 
the mechanisms underlying the pruning and selection on one of the ribbon-occupied synaptic contacts are unclear.

\subsection{The synaptic $\mathrm{Ca}^{2+}$ signal and its developmental increase in heterogeneity}

As discussed above, the IHC assumes different roles during murine postnatal development. The switch in electric activity and synaptic morphology prompted the question whether the $\mathrm{Ca}^{2+}$ signal at the presynaptic $\mathrm{AZ}$, the trigger for exocytosis of vesicles, changes according to the function of IHCs. In this study, I inspected the properties of synaptic $\mathrm{Ca}^{2+}$ signal in IHCs before and after the onset of hearing using high resolution confocal imaging, and attempted to compare them both quantitatively and under naturalistic situation at each age.

\subsubsection{AP-associated $\mathrm{Ca}^{2+}$ transients and their potential functions}

A recent study (Johnson et al., 2012), has demonstrated $\mathrm{Ca}^{2+}$ transients associated with single AP, using line scans and a high affinity dye Fluo-4 [dissociation constant $\left(\mathrm{K}_{\mathrm{d}}\right)=345 \mathrm{nM}$; Molecular Probes]. They were able to report a rather global increase across the basal part of the IHC. However, the high affinity dye would be saturated at the micromolar range of $\left[\mathrm{Ca}^{2+}\right]$ required for triggering exocytosis in IHC (Beutner et al., 2001). Using a low affinity dye (Fluo-4FF, $\mathrm{K}_{\mathrm{d}}=9.7 \mu \mathrm{M}$; Molecular Probes), I was also able to observe $\mathrm{Ca}^{2+}$ transients at synaptic AZ during single APs in pre-hearing IHCs (Figure 3.1). The high $\mathrm{K}_{\mathrm{d}}$ of Fluo-4FF allows a quantitative comparison of the synaptic $\mathrm{Ca}^{2+}$ signal during an action potential. I demonstrated that the synaptic $\mathrm{Ca}^{2+}$ signal during an AP is of similar amplitude to that of a step depolarization to $-7 \mathrm{mV}$, further supporting the notion that AP in pre-hearing IHCs can trigger release of glutamate (Beutner and Moser, 2001; Brandt et al., 2003; Johnson et al., 2005).

Besides triggering vesicle release and consequently driving bursts of AP in afferent SGNs, the $\mathrm{Ca}^{2+}$ influx in pre-hearing IHCs promotes cell development (Brandt et al., 2003; Neef et al., 2009). In knockout mouse of the pore forming subunit $\alpha_{1 \mathrm{D}}$ of Cav1.3 channels (Brandt et al., 2003; Nemzou et al., 2006), or a genetic deletion of the auxiliary subunit $\beta_{2}$ (Neef et al., 2009), $\mathrm{Ca}^{2+}$ current in IHC was significantly reduced and a mixture of immature and mature phenotype was observed even after the age of hearing onset in wildtype. For instance, in both cases IHCs showed a persistence of efferent innervation and a significant reduction or lack of BK channel expression, both an immature phenotype. On the other hand, the developmental 
upregulation of KCNQ channels was observed in both mutants similar to mature wildtype IHCs. This indicates that the $\mathrm{Ca}^{2+}$ influx evoked by spontaneous AP in pre-hearing IHC is probably important for a subset of developmental changes, while other aspects of IHC proceed relatively independent of such $\mathrm{Ca}^{2+}$ signals It would be interesting to see how subtler disruption of $\mathrm{Ca}^{2+}$-APs in IHCs, e.g. knockout of voltage-gated $\mathrm{Na}^{+}$channels (Raouf et al., 2012) or overexpression of SK2 channels (Maison et al., 2007), changes the IHC maturation program.

\subsubsection{Ability of mature synaptic $\mathrm{Ca}^{2+}$ signaling to follow high frequency}

One of the most impressive feats of the auditory system is their ability to encode temporal fine structure of a sound. The ability of auditory nerve fibers to phase-lock to the stimulus, has been shown in multiple mammalian species, including cats (Johnson, 1980), guinea pigs (Palmer and Russell, 1986), squirrel monkeys (Rose et al., 1967) and mice (Taberner and Liberman, 2005). The reliability of phase-locking is important for various computation in central auditory system, for example in sound source localization (Köppl, 2009; Grothe et al., 2010) or in pitch perception (Reichenbach and Hudspeth, 2012). Given that auditory nerve fibers receive excitatory input exclusively from IHCs (see IntroductionIntroduction), the release of neurotransmitter from an IHC should also be able to follow stimuli at frequencies for which phase-locking is observed. In fact, a recent study using pair-recordings of IHC and afferent bouton terminal (Goutman, 2012) showed that release of the IHC ribbon synapse could phase-lock to a train of periodic depolarization, relatively insensitive to stimulus amplitude.

I investigated the synaptic $\mathrm{Ca}^{2+}$ signal of mature IHCs under receptor-potential-like stimuli, and was able to observe oscillation of $\mathrm{Ca}^{2+}$ signal up to $1 \mathrm{kHz}$ despite attenuation. The ability of the release of an IHC to follow high frequency stimuli is limited by filtering properties of processes during sensory transduction, namely mechanoelectrical transduction, membrane capacitance, channel activation kinetics and diffusion-buffering of $\mathrm{Ca}^{2+}$ ions. Ultimately, the binding of $\mathrm{Ca}^{2+}$ to the $\mathrm{Ca}^{2+}$ sensor of release and the rate of the fusion process further limit the bandwidth.

The adaptation of mechanoelectrical transduction mainly acts as a high pass filter (Jia et al., 2007) and is experimentally bypassed during whole-cell patch-clamp recordings. Downstream 
to transduction current, the plasma membrane of an IHC acts as a low pass filter (Figure 3.3 and Figure 3.4). In the current clamp experiments, pre-hearing (p10) IHCs typically have a membrane resistance $\left(\mathrm{R}_{\mathrm{m}}\right)$ of around $500 \mathrm{M} \Omega$ at $-87 \mathrm{mV}$, which declines to around $100 \mathrm{M} \Omega$ in p14-17 IHCs, probably due to expression of potassium conductances (Kros et al., 1998; Oliver et al., 2003). $R_{m}$ and the membrane capacitance $\left(C_{m}\right)$ combined determine the characteristics of the low-pass filter, with the membrane time constant equals to the product $\mathrm{R}_{\mathrm{m}} \mathrm{C}_{\mathrm{m}}$. As shown earlier (Figure 3.4 and Table 1), I observed higher cutoff frequencies at more positive holding potentials, and at larger stimulus amplitude. This was probably due to the activation of $\mathrm{K}^{+}$conductances, which lowered $\mathrm{R}_{\mathrm{m}}$ and thus reducing the membrane time constant (higher cutoff). Interestingly, as demonstrated in Figure 4.4, a lower $\mathrm{R}_{\mathrm{m}}$ will not help increasing a.c. response amplitude, which is important for frequency-modulated activation of $\mathrm{Ca}^{2+}$ channels. The phase lag of the response slightly decreased at any given frequency, which may not be critical for phase-locking in of release in IHCs as long as the phase is constant. However, a closely controlled phase-shift might be essential for the function of OHCs, which need to provide power output for sound amplification.

In addition to filtering due to the RC-circuit of the membrane, the activation and deactivation of $\mathrm{Ca}^{2+}$ channels also have finite kinetics (Nouvian, 2007; Johnson and Marcotti, 2008; Zampini et al., 2010) and will not be able to follow high frequency changes in membrane potential. This is indeed observed in experiments shown in Figure 3.5c, where the $\mathrm{Ca}^{2+}$ current was attenuated at higher frequencies.

Last but not least, the observed $\mathrm{Ca}^{2+}$ signal shown in Figure 1.3 is likely an underestimate of the underlying free $\left[\mathrm{Ca}^{2+}\right.$, due to (1) limited binding kinetics of the $\mathrm{Ca}^{2+}$ indicator Fluo-4FF $\left(\mathrm{k}_{\mathrm{on}}=140 \mu \mathrm{M}^{-1} \mathrm{~s}^{-1} ; \mathrm{k}_{\mathrm{off}}=1400 \mathrm{~s}^{-1} ;\right.$ Murat Alp, personal communication), and (2) spatial averaging of fluorescence signal. Even assuming a relatively high average $\left[\mathrm{Ca}^{2+}\right]$ of $10 \mu \mathrm{M}$, which increases the overall reaction rate, the time constant $\tau$ for the binding reaction will be approximately $0.36 \mathrm{~ms}$. This gives a cutoff frequency $f_{0}$ of $445 \mathrm{~Hz}\left(f_{0}=1 / 2 \pi \tau\right)$ and may explain the decline of $\mathrm{Ca}^{2+}$ signal modulation at high frequency. Spatial averaging occurs because the point spread function of the confocal system is diffraction limited $(\sim 0.30 \mu \mathrm{m}$ FWHM in lateral directions, $\sim 1.25 \mu \mathrm{m}$ in axial direction, Frank et al., 2009). The further away from the site of $\mathrm{Ca}^{2+}$ influx, the smaller change in $\left[\mathrm{Ca}^{2+}\right]$ would be induced due to diffusion 
itself and additional time for binding by slower buffers (Naraghi and Neher, 1997). Therefore the average fluorescence from the confocal point spread function may underestimate the $\left[\mathrm{Ca}^{2+}\right]$ at synaptic vesicles, which locate in nanometer proximity to $\mathrm{Ca}^{2+}$ channels (Brandt et al., 2005).

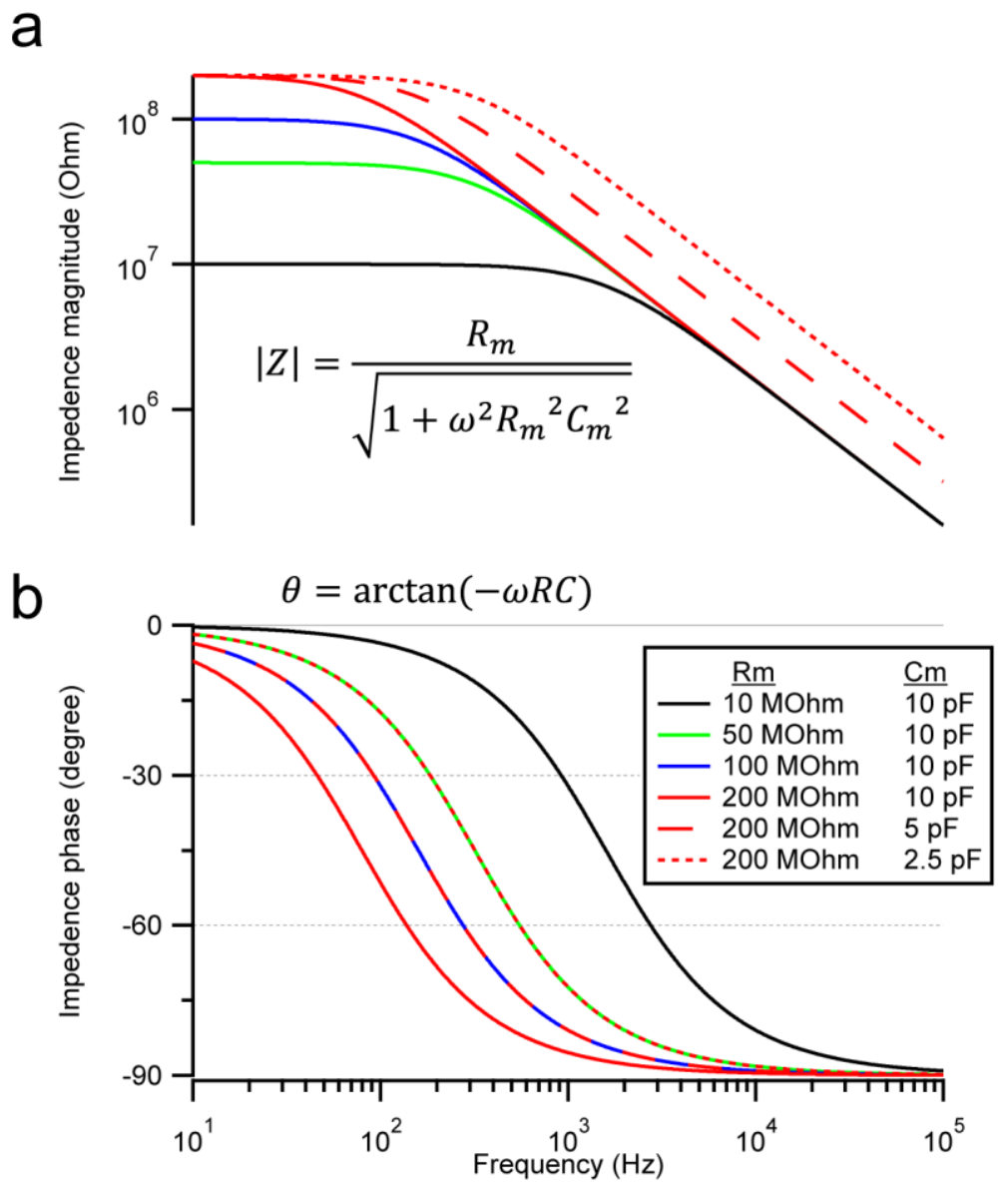

\section{Figure 4.4: Effects of $R_{m}$ and $C_{m}$ on low-pass filtering}

(a) The magnitude, $|Z|$, of the impedance in a parallel connection of a resistor $R_{m}$ and a capacitor $C_{m}$, versus frequency, calculated with the equation in the figure. In the equation, $\omega$ is the angular frequency in $\operatorname{rad~s}^{-1}$.Lower $R_{m}$ reduces $|Z|$ at low frequency and increases the cutoff frequency, but has no effect at high frequencies. A decrease in $C_{m}$ increases $|Z|$ at high frequencies and also increases the cutoff frequency, but does not affect $|Z|$ at low frequencies (b) Phase shift of the response with respect to the input, calculated by the equation on the figure. 


\subsubsection{Quantitative measurement of synaptic $\mathrm{Ca}^{2+}$ signal and possible errors}

The quantitative measurement of the synaptic $\mathrm{Ca}^{2+}$ signal, might have been biased towards synapses with smaller $\Delta \mathrm{F}$ values in pre-hearing IHCs because $\mathrm{I}$ tried to avoid $\mathrm{Ca}^{2+}$ microdomains with two nearby ribbons. These $\mathrm{Ca}^{2+}$ microdomains could represent synapses with multiple ribbons as indicated by images in Figure 3.13 and Figure 3.14. Figure 3.14We do not know whether the developmental decrease in ribbon number represents the pruning of extra ribbons/synapses or the conglomeration of ribbons at the same IHC-SGN contact. If the latter case is true, then the AZs with strong $\mathrm{Ca}^{2+}$ influx emerged after the onset of hearing (Figure 3.10) could already possess a larger $\mathrm{Ca}^{2+}$ channel complement.

Another possible source of experimental variability comes from the position where the $\mathrm{Ca}^{2+}$ indicator fluorescence was sampled. Stronger change in indicator fluorescence can be observed closer to the center of the $\mathrm{Ca}^{2+}$ channel cluster (Frank et al., 2009). Line-scans were preferentially used in this study over point scans to minimize the error due to lateral misalignment of the $\mathrm{Ca}^{2+}$ microdomain and the position of measurement. Misalignment in the axial position can also occur, possibly contributed to the negative correlation observed between the FWHM and the amplitude of the $\mathrm{Ca}^{2+}$ signal (Figure 3.11a). However, such correlation was observed in both age group, and when data points with large FWHM $(>1 \mu \mathrm{m})$ were removed, the ratio of mean $\Delta \mathrm{F}$ amplitude remained unchanged (p10:p14-16; from 0.82 to 0.82$)$.

The size of the synaptic ribbon may also affect the quantitative measurement of $\mathrm{Ca}^{2+}$ influx. Previous report have suggested an enrichment of a related $\mathrm{Ca}^{2+}$ indicator Fluo-3 at the synaptic ribbon (Issa and Hudspeth, 1996). Although I did not observe an obvious increase of baseline fluorescence $\left(\mathrm{F}_{0}\right)$ at the ribbon (Figure 4.5), any effect of higher dye concentration should be minimized by normalization to $F_{0}$ (Figure 3.10d, f). Alternatively, the synaptic ribbon was suggested to function as a physical diffusion barrier for $\mathrm{Ca}^{2+}$ ion, providing a mechanism for high $\left[\mathrm{Ca}^{2+}\right]$ and synchronous multivesicular release (Graydon et al., 2011). In the framework of this hypothesis and given the developmental increase in ribbon size (Figure 3.14 and Table 2), the observed increase in $\mathrm{Ca}^{2+}$ signal amplitude may not directly reflect increase in $\mathrm{AZ} \mathrm{Ca}{ }^{2+}$ influx, but could instead be partly contributed by stronger spatial confinement under the ribbon. However, the qualitative interpretation of stronger $\mathrm{Ca}^{2+}$ signal 
mediating stronger synapse would still hold, as the increase in multivesicular release should give rise to larger and more monophasic EPSCs, which increases the probability of spike generation at the postsynaptic SGN (Grant et al., 2010; Rutherford et al., 2012).
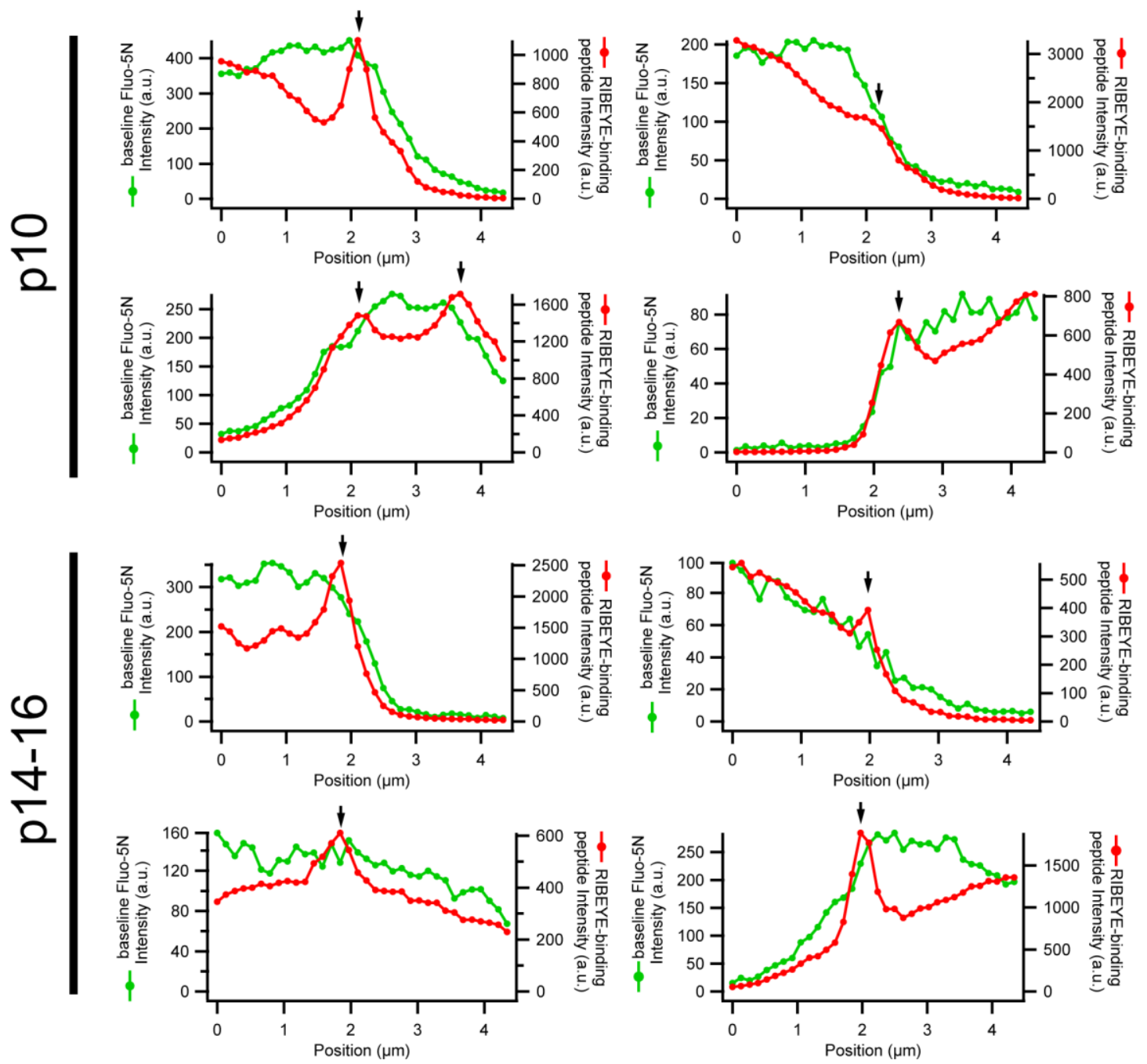

\section{Figure 4.5: Comparison of RIBEYE-binding peptide intensity and baseline fluorescence of Fluo-5N}

Average baseline fluorescence of Fluo-5N (green) and TAMRA-conjugated RIBEYE-binding peptide (monomer, red) for several example line scans. Arrows mark positions of identified ribbons. No obvious enrichment of Fluo-5N baseline fluorescence was observed.

\subsubsection{Presynaptic heterogeneity as a mechanism to decompose auditory information}

As discussed earlier, SGNs differ in sound-sensitivity and dynamic range and are thought to jointly encode the large dynamic range spanning six orders of magnitude in sound pressure (reviewed in Meyer and Moser, 2010). While an IHC is thought to be contacted by 5-30 SGN 
afferents with different spontaneous rates and thresholds, all AZs of the small isopotential IHC experience the same membrane potential. Here, I confirmed the previously reported heterogeneity of $\mathrm{Ca}^{2+}$ signal strength among IHC synapses (Frank et al., 2009), and found a developmental increase of variability through the gain of $\mathrm{AZ}$ with stronger $\mathrm{Ca}^{2+}$ influx. Previous measurements in the early postnatal cat, another altricial species, have also reported an emergence of high spontaneous rate auditory nerve fibers, which functionally correlate with higher sensitivity (Walsh and McGee, 1987). I propose that AZ with a higher number of $\mathrm{Ca}^{2+}$ channel and, hence, stronger $\mathrm{Ca}^{2+}$ signal, drive SGNs with higher spontaneous rate and sound sensitivity.

How could a difference in $\mathrm{Ca}^{2+}$ channel number affect synaptic strength? Assuming a tight coupling of $\mathrm{Ca}^{2+}$ channel to vesicle in the IHC (Figure 3.18 and Brandt et al., 2005), a larger $\mathrm{AZ}$ would possess more functional release units, each composed of $\mathrm{Ca}^{2+}$ channel(s) and a release ready vesicle. That will increase the chance of an AZ to release any vesicle during a stimulus even with a constant release probability. This is consistent with a higher spontaneous rate postsynaptic neuron. In addition, an recent study by Sheng and colleagues (2012) in the calyx of Held has demonstrated that vesicle release probability at individual AZ increases with $\mathrm{Ca}^{2+}$ channel number, providing evidence that the strength of an $\mathrm{AZ}$ can be controlled by its channel complement.

While the discussion above has been focusing on the magnitude of $\mathrm{Ca}^{2+}$ influx, other mechanisms cannot be excluded. For instance, differences in voltage-dependence of synaptic $\mathrm{Ca}^{2+}$ influx have been demonstrated in this study (Figure 3.10) and previously (Frank et al., 2009). This could give rise to variability in sensitivity of AZ to IHC receptor potential, as $\mathrm{Ca}^{2+}$ channels with more hyperpolarized $\mathrm{V}_{\text {half }}$ will be activated by a smaller depolarization, giving the $\mathrm{AZ}$ a higher sensitivity. Evidence from postsynaptic recording have also shown that SGNs can receive different modes of release (multiphasic or monophasic EPSCs) in variable proportion (Grant et al., 2010). In addition, differences in postsynaptic properties, such as efferent modulation (Ruel et al., 2001; Puel et al., 2002), number of glutamate receptors (Liberman et al., 2011) and its long-term adaptation (Chen et al., 2009), may co-exist and contribute to fine tuning the sensitivity of the auditory system. 


\subsubsection{Possible mechanisms mediating presynaptic heterogeneity}

If the difference in $\mathrm{Ca}^{2+}$ channel number among $\mathrm{AZ}$ of an IHC is biologically relevant, one would ask how such heterogeneity is achieved mechanistically and how a cell controls the channel distribution among synapses. It is tempting to speculate that the determination of $\mathrm{Ca}^{2+}$ channel complement is mediated through interaction of presynaptic scaffold and/or transsynaptic signaling.

Presynaptically, the synaptic ribbon appears to stabilize the clustering of $\mathrm{Ca}^{2+}$ channel at the IHC active zone (Frank et al., 2010), and a correlation between intensity of RIBEYE-binding peptide and amplitude $\mathrm{Ca}^{2+}$ signal was indicated (Frank et al., 2009). Unfortunately in the smaller prehearing IHCs, the proximity of nuclei, which is also labeled by the monomeric peptide, precluded such quantification in the current study. A study on zebrafish lateral line hair cells has reported that manipulation of RIBEYE expression level leads to a disruption of $\mathrm{Ca}^{2+}$ channel abundance and localization (Sheets et al., 2011). This interaction appeared to be bidirectional and rather dynamic, as the activity of $\mathrm{Ca}_{\mathrm{V}} 1.3$ channels also affects ribbon size (Sheets et al., 2012). Mutants of presynaptic scaffold protein Bassoon (Khimich et al., 2005; Frank et al., 2010), and an Usher-syndrome related protein Harmonin (Gregory et al., 2011), were also shown to affect the expression level of $\mathrm{Ca}^{2+}$ channel. In particular, disruption of Bassoon in IHCs reduced the number of $\mathrm{Ca}^{2+}$ channels and their ultrastructural organization at the AZ (Frank et al., 2010). Although Bassoon was shown not to directly interact with Cav1.3 channels in a heterologous expression system, its effect may have mediated through the disruption of ribbons (Khimich et al., 2005; Frank et al., 2010) or other components of the cytomatrix of active zone (Gundelfinger and Fejtova, 2012), such as Rab3-interacting molecule (RIM). On the other hand, Harmonin, a putative interaction partner of Cav1.3 channel, was found to be present only in a subset of IHC ribbon synapses, with a developmental increase in the number of Harmonin-positive synapses (Gregory et al., 2011). In the same study, $\mathrm{Ca}^{2+}$ imaging reported an increase in the average synaptic $\mathrm{Ca}^{2+}$ influx in Harmonin mutant, suggesting that the presence of Harmonin may function to limit the recruitment of $\mathrm{Ca}_{\mathrm{V}} 1.3$ to a subset of AZs. However, such hypotheses simply push the question further up the regulatory pathway, to the mechanism by which these presynaptic AZ proteins were differentially distributed. Future experiments should also address the possibility of transsynaptic signaling and influence of trophic factors. 
Alternatively, one might presume a self-organization of $\mathrm{AZ}$, in which newly synthesized $\mathrm{Ca}^{2+}$ channel are preferentially attached to larger AZ, while a similar rate of degradation applies across all AZs. This is reminiscence of a preferential attachment model in the study of internet network topology, in which a power-law distribution of cluster sizes was observed (Barabási and Albert, 1999). However, the distributions of $\Delta \mathrm{F}$ amplitudes observed in the current study (Figure 3.12) were actually better approximated by log-normal distributions. A study on dendritic spines observed that their sizes change dynamically, while maintaining a log-normal distribution as a population (Loewenstein et al., 2011). Regrettably, the model by Loewenstein and colleagues (2011) did not provide insight to the mechanism in maintaining such a dynamic equilibrium.

\subsection{Utility of vGpH in reporting vesicle recycling in IHCs}

To further relate presynaptic heterogeneity observed in IHC synaptic $\mathrm{Ca}^{2+}$ influx, I attempted to use an optical probe of exocytosis $\mathrm{vGpH}$ to measure vesicle fusion at an individual $\mathrm{AZ}$ level. In the following, utility of this reporter in IHC will be discussed and suggestions on further optimization or alternatives will be given.

\subsubsection{Extensive subcellular localization of vGpH in transduced IHCs}

Unlike in cultured hippocampal neurons where $\mathrm{vGpH}$ is specifically targeted in presynaptic terminals (Voglmaier et al., 2006), both live-imaging and immunohistochemistry reveals a rather ubiquitous subcellular localization of the protein (see section 0). However, the expression pattern of $\mathrm{vGpH}$ was well correlated with the endogenous glutamate transporter vglut3 (Figure 3.20). The apparent ambiguity may stem from the intrinsic protein trafficking scheme in the IHC, which lacks compartmental specialization of the presynapse. In the central nervous system, vglut3 is found in soma and dendrite of both glutamatergic and nonglutamertergic neurons, as well as astrocytes (reviewed in Benarroch, 2010). The nonsynaptic localization calls for a function different from loading of synaptic vesicles. The same could be true for IHC, and not all vglut3-containing organelles in the IHC may be synaptic vesicles.

Nevertheless, live imaging of $\mathrm{vGpH}$ expressing IHCs showed depolarization-evoked increase in fluorescence, which specifically localized at the AZ (Figure 3.24). This shows that at least a subset of $\mathrm{vGpH}$ containing organelles was able to participate in exocytosis, and potentially 
contribute to glutamate release. The $\mathrm{vGpH}$ around the nucleus level of transduced IHCs seemed to reside in less acidic organelle ( $\sim \mathrm{pH} 6.5$, Figure 3.23$)$ as compared to the vesicle lumen in neuronal synapses (pH 5.5, Miesenböck et al., 1998). Rather, similar pH have been reported in the Golgi apparatus, using synthetic dyes in HeLa cells (pH 6.4, Wu et al., 2000) or ratiometric pHluorin in neurons and keratinocytes (pH 6.2, Miesenböck et al., 1998; pH 6.3, Disbrow et al., 2005). However, co-staining of cis-Golgi-network with anti-GM130 antibody showed an apparent exclusion of the $\mathrm{vGpH}$ (Figure 3.22). The identity of organelles housing vGpH remains elusive, and they may not necessarily be a homogenous population.

\subsubsection{Signal-to-noise ratio for vGpH imaging}

One advantage of vGpH confers in neuron, over the smaller synaptopHluorin (Miesenböck et al., 1998; Sankaranarayanan et al., 2000), is its higher signal-to-noise ratio due to less surface expression (Voglmaier et al., 2006; Balaji and Ryan, 2007). In this regard, the relatively broad intracellular expression of $\mathrm{vGpH}$ in IHCs (Figure 3.20) would mitigate this advantage simply by having higher intracellular baseline fluorescence.

Unfortunately, the confocal imaging system used in this study did not provide a good signalto-noise ratio, even with high laser intensity incurring significant amount of bleaching (Figure 3.25). The fact that the pHluorin signal was dominated by Poisson shot noise precludes improvement of signal to noise by increase amplification at the detector level. Instead, signal has to be optimized by increasing the number of detectable photons. Usually, this can be achieved by either increase emission from the fluorophore or improve the collection of emitted photons. Since significant bleaching already occurs in the current setting, the excitation laser power cannot be increased further. That leaves the problem to (1) reducing the loss of photons through the imaging system or (2) using a detector with higher quantum yield. Alternatively, switching to a two-photon excitation system may improve this situation because it removes the need of a detection pinhole, which normally blocks majority of emitted photons.

\subsubsection{Alternative optical reporters for single $\mathrm{AZ}$ release}

A red-shifted $\mathrm{pH}$-sensitive fluorescent protein mOrange2 has been recently used for measuring exocytosis in combination with $\mathrm{Ca}^{2+}$ imaging (Hires et al., 2011). In this study, the pHluorin in $\mathrm{vGpH}$ construct were replaced by two tandem copies of mOrange2, and the resulting fusion protein was used in combination with simultaneous $\mathrm{Ca}^{2+}$ imaging with 
another genetically-encoded reporter SyGCaMP3. The use of a red-shifted protein may be advantageous for IHCs as concurrent $\mathrm{Ca}^{2+}$ imaging was one of the major goal. However, one should consider whether the other vesicular protein should be used, given the widespread subcellular localization of $\mathrm{vGpH}$ (Figure 3.20). Since the IHC synapse lacks many of the neuronal vesicular markers (see Introduction, p6), finding a protein specifically localized to synaptic vesicle may prove difficult. Otoferlin, a putative $\mathrm{Ca}^{2+}$ sensor for release in IHC, has a relatively strong membrane expression (Figure 3.21b). If the membrane localization represents surface expression, it may be detrimental to signal-to-noise ratio for pHluorin imaging as discussed for synaptopHluorin (see above and Voglmaier et al., 2006; Balaji and Ryan, 2007; Zhu et al., 2009).

Dextran-coupled fluorophores are another alternative to FM dyes (for their problems, see section 1.4) for labeling recycling vesicles, as demonstrated in a recent study on rod photoreceptors (Chen et al., 2013). Similarly to FM-dyes, the dextran-coupled dye is first applied externally in conditions where vesicle recycling occurs, and the unloading of labeled vesicles is used as an indication of exocytosis. This method has the advantage of selectively labeling vesicles undergoing active recycling, potentially circumventing the baseline fluorescence from possible non-synaptic organelle observed in $\mathrm{vGpH}$ expressing IHCs.

Other than vesicle fusion, the release of neurotransmitter can also be monitored. Recently, genetically-encoded sensor for glutamate have been developed (Okumoto et al., 2005; Hires et al., 2008; Marvin et al., 2013). In particular, the functionality of single fluorophore version iGluSnFR in neurons was demonstrated in worm, zebrafish and mice in vivo (Marvin et al., 2013). One property of using glutamate imaging instead of pHluorin, is that it could uncouple signal of vesicle exocytosis from membrane-stranded vesicular protein, which are retrieved in a time scale of seconds in IHCs (Moser and Beutner, 2000; Pangršič et al., 2010) and in neurons (Balaji and Ryan, 2007; Zhu et al., 2009). It would be, however, unsuitable for observing endocytosis of vesicles, and the expression and correct targeting of such reporter in SGNs have yet to be tested. 


\section{Summary}

Hearing over a wide range of sound intensities is thought to require complementary coding by functionally diverse spiral ganglion neurons (SGNs), each changing its firing rate only over a limited sub-range. The means for establishing SGN diversity are not well understood, but likely involves differences among their inputs - the presynaptic active zones (AZs) of inner hair cells (IHCs). In this study, I investigated the $\mathrm{Ca}^{2+}$ signal in IHCs, which were previously demonstrated to be heterogeneous among AZs in mature IHCs. In particular, I explored the changes of synaptic $\mathrm{Ca}^{2+}$ signal during postnatal development of the mouse cochlea, and probed the functional consequence thereof. Moreover, I attempted to establish analogous measurement of exocytosis using the $\mathrm{pH}$-sensitive fluorescent protein pHluorin.

The results showed that depolarization-evoked $\mathrm{Ca}^{2+}$ signal in IHC became progressively confined to AZs during development, mostly mediated by a loss of extrasynaptic channels revealed by immunohistochemistry of $\mathrm{Ca}_{v} 1.3$ channels. Parallel to the confinement of $\mathrm{Ca}^{2+}$ channel, the IHC switches from a $\mathrm{Ca}^{2+}$ microdomain control of exocytosis to a tighter $\mathrm{Ca}^{2+}$ nanodomain control, without a change in intrinsic $\mathrm{Ca}^{2+}$ dependence of vesicle fusion.

At the level of individual AZs, $\mathrm{Ca}^{2+}$ action potentials in prehearing IHCs drove robust $\mathrm{Ca}^{2+}$ transients. On the other hand, receptor-potential-like stimuli were able to drive synaptic $\mathrm{Ca}^{2+}$ signals in mature IHCs up to $1 \mathrm{kHz}$, even given experimental limitations. An increase in heterogeneity of synaptic $\mathrm{Ca}^{2+}$ influx among the AZs of IHCs was observed, mediated by the strengthening of $\mathrm{Ca}^{2+}$ signaling at a subset of AZs through a gain of $\mathrm{Ca}^{2+}$ channels. The emergence of SGN with high spontaneous rate around the onset of hearing suggests that $\mathrm{AZ}$ with stronger $\mathrm{Ca}^{2+}$ influx drive these postsynaptic SGNs which are more sensitive to sound. However, other mechanisms including differences in voltage-dependence of $\mathrm{Ca}^{2+}$ channel, number of postsynaptic glutamate receptors and efferent modulation cannot be excluded.

Depolarization of mature IHCs expressing vglut1-pHluorin revealed localized exocytic activity at the AZs, similar to the confined $\mathrm{Ca}^{2+}$ signal. However, the signal-to-noise ratio is yet to be improved for quantitative measurement of exocytosis. The establishment of a single $\mathrm{AZ}$ measurement of vesicle release will help to confirm whether the heterogeneity of synaptic $\mathrm{Ca}^{2+}$ signal indeed lead to difference in output. 


\section{References:}

Augustine GJ, Adler EM, Charltonc MP (1991) The calcium signal for transmitter secretion from presynaptic nerve terminals. Ann N Y Acad Sci 635:365-381.

Balaji J, Ryan TA (2007) Single-vesicle imaging reveals that synaptic vesicle exocytosis and endocytosis are coupled by a single stochastic mode. Proc Natl Acad Sci U S A 104:20576-20581.

Barabási A-L, Albert R (1999) Emergence of scaling in random networks. Science 286:509512.

Batteau DW (1967) The role of the pinna in human localization. Proc R Soc Lond B Biol Sci 168:158-180.

Benarroch EE (2010) Glutamate transporters: diversity, function, and involvement in neurologic disease. Neurology 74:259-264.

Bertram R, Sherman A, Stanley EF (1996) Single-domain/bound calcium hypothesis of transmitter release and facilitation. J Neurophysiol 75:1919-1931.

Beurg M, Michalski N, Safieddine S, Bouleau Y, Schneggenburger R, Chapman ER, Petit C, Dulon D (2010) Control of exocytosis by synaptotagmins and otoferlin in auditory hair cells. J Neurosci 30:13281 -13290.

Beutner D, Moser T (2001) The presynaptic function of mouse cochlear inner hair cells during development of hearing. J Neurosci 21:4593.

Beutner D, Voets T, Neher E, Moser T (2001) Calcium dependence of exocytosis and endocytosis at the cochlear inner hair cell afferent synapse. Neuron 29:681-690.

Blankenship AG, Feller MB (2010) Mechanisms underlying spontaneous patterned activity in developing neural circuits. Nat Rev Neurosci 11:18-29.

Bollmann JH, Sakmann B, Borst JGG (2000) Calcium sensitivity of glutamate release in a calyx-type terminal. Science 289:953-957.

Borst JGG, Sakmann B (1996) Calcium influx and transmitter release in a fast CNS synapse. Nature 383:431-434.

Brandt A, Khimich D, Moser T (2005) Few Cav1.3 channels regulate the exocytosis of a synaptic vesicle at the hair cell ribbon synapse. J Neurosci 25:11577.

Brandt A, Striessnig J, Moser T (2003) CaV1.3 channels are essential for development and presynaptic activity of cochlear inner hair cells. J Neurosci 23:10832.

Brown MB, Forsythe AB (1974) Robust tests for the equality of variances. J Am Stat Assoc 69:364-367. 
Bulankina AV, Moser T (2012) Neural circuit development in the mammalian cochlea. Physiology 27:100-112.

Chen M, Van Hook MJ, Zenisek D, Thoreson WB (2013) Properties of ribbon and non-ribbon release from rod photoreceptors revealed by visualizing individual synaptic vesicles. $\mathrm{J}$ Neurosci 33:2071-2086.

Chen Z, Peppi M, Kujawa SG, Sewell WF (2009) Regulated expression of surface AMPA receptors reduces excitotoxicity in auditory neurons. J Neurophysiol 102:1152-1159.

Collingridge GL, Olsen RW, Peters J, Spedding M (2009) A nomenclature for ligand-gated ion channels. Neuropharmacology 56:2-5.

Cui G, Meyer AC, Calin-Jageman I, Neef J, Haeseleer F, Moser T, Lee A (2007) Ca2+binding proteins tune $\mathrm{Ca} 2+-$ feedback to Cav1.3 channels in mouse auditory hair cells. J Physiol 585:791-803.

Cull-Candy S, Kelly L, Farrant M (2006) Regulation of Ca2+-permeable AMPA receptors: synaptic plasticity and beyond. Curr Opin Neurobiol 16:288-297.

Dallos P (1985) Membrane potential and response changes in mammalian cochlear hair cells during intracellular recording. J Neurosci 5:1609-1615.

Dean C, Liu H, Dunning FM, Chang PY, Jackson MB, Chapman ER (2009) SynaptotagminIV modulates synaptic function and LTP by regulating BDNF release. Nat Neurosci $12: 767-776$.

Defourny J, Lallemend F, Malgrange B (2011) Structure and development of cochlear afferent innervation in mammals. Am J Physiol Cell Physiol 301:C750-C761.

Demandolx D, Davoust J (1997) Multicolour analysis and local image correlation in confocal microscopy. J Microsc 185:21-36.

Dick O, Dieck S tom, Altrock WD, Ammermüller J, Weiler R, Garner CC, Gundelfinger ED, Brandstätter JH (2003) The presynaptic active zone protein bassoon is essential for photoreceptor ribbon synapse formation in the retina. Neuron 37:775-786.

DiGregorio DA, Vergara JL (1997) Localized detection of action potential-induced presynaptic calcium transients at a Xenopus neuromuscular junction. J Physiol 505:585-592.

Disbrow GL, Hanover JA, Schlegel R (2005) Endoplasmic reticulum-localized human papillomavirus type $16 \mathrm{E} 5$ protein alters endosomal $\mathrm{pH}$ but not trans-Golgi $\mathrm{pH}$. J Virol 79:5839-5846.

Donnelly SM, Kramer A (1999) Testing for multiple species in fossil samples: An evaluation and comparison of tests for equal relative variation. Am J Phys Anthropol 108:507529 . 
Dou H, Vazquez AE, Namkung Y, Chu H, Cardell EL, Nie L, Parson S, Shin H-S, Yamoah EN (2004) Null mutation of alpha1D Ca2+ channel gene results in deafness but no vestibular defect in mice. J Assoc Res Otolaryngol 5:215-226.

Dreosti E, Lagnado L (2011) Optical reporters of synaptic activity in neural circuits. Exp Physiol 96:4-12.

El Barbary A (1991) Auditory nerve of the normal and jaundiced rat. I. Spontaneous discharge rate and cochlear nerve histology. Hear Res 54:75-90.

Eybalin M, Caicedo A, Renard N, Ruel J, Puel J-L (2004) Transient Ca2+-permeable AMPA receptors in postnatal rat primary auditory neurons. Eur J Neurosci 20:2981-2989.

Faas GC, Raghavachari S, Lisman JE, Mody I (2011) Calmodulin as a direct detector of Ca2+ signals. Nat Neurosci 14:301-304.

Fedchyshyn MJ, Wang L-Y (2005) Developmental Transformation of the release modality at the calyx of Held synapse. J Neurosci 25:4131-4140.

Fernandez-Alfonso T, Ryan TA (2008) A heterogeneous "resting" pool of synaptic vesicles that is dynamically interchanged across boutons in mammalian CNS synapses. Brain Cell Bio 36:87-100.

Fiala JC (2005) Reconstruct: a free editor for serial section microscopy. J Microsc 218:52-61.

Francis AA, Mehta B, Zenisek D (2011) Development of new peptide-based tools for studying synaptic ribbon function. J Neurophysiol 106:1028-1037.

Frank T, Khimich D, Neef A, Moser T (2009) Mechanisms contributing to synaptic Ca2+ signals and their heterogeneity in hair cells. Proc Natl Acad Sci U S A 106:4483-4488.

Frank T, Rutherford MA, Strenzke N, Neef A, Pangrsic T, Khimich D, Fetjova A, Gundelfinger ED, Liberman MC, Harke B, Bryan KE, Lee A, Egner A, Riedel D, Moser T (2010) Bassoon and the synaptic ribbon organize $\mathrm{Ca} 2+$ channels and vesicles to add release sites and promote refilling. Neuron 68:724-738.

Fuchs PA (2005) Time and intensity coding at the hair cell's ribbon synapse. J Physiol 566:712.

Gaffield MA, Betz WJ (2007) Imaging synaptic vesicle exocytosis and endocytosis with FM dyes. Nat Protoc 1:2916-2921.

Gale JE, Marcotti W, Kennedy HJ, Kros CJ, Richardson GP (2001) FM1-43 dye behaves as a permeant blocker of the hair-cell mechanotransducer channel. J Neurosci 21:7013.

Geng R, Melki S, Chen DH-C, Tian G, Furness DN, Oshima-Takago T, Neef J, Moser T, Askew C, Horwitz G, Holt JR, Imanishi Y, Alagramam KN (2012) The 
Mechanosensory Structure of the hair cell requires clarin-1, a protein encoded by Usher syndrome III causative gene. J Neurosci 32:9485-9498.

Glowatzki E, Fuchs PA (2000) Cholinergic synaptic inhibition of inner hair cells in the neonatal mammalian cochlea. Science 288:2366.

Glowatzki E, Fuchs PA (2002) Transmitter release at the hair cell ribbon synapse. Nat Neurosci 5:147-154.

Goda Y, Davis GW (2003) Mechanisms of synapse assembly and disassembly. Neuron 40:243-264.

Goutman JD (2012) Transmitter release from cochlear hair cells is phase locked to cyclic stimuli of different intensities and frequencies. J Neurosci 32:17025-17036.

Goutman JD, Glowatzki E (2007) Time course and calcium dependence of transmitter release at a single ribbon synapse. Proc Natl Acad Sci U S A 104:16341.

Goutman JD, Glowatzki E (2011) Short-term facilitation modulates size and timing of the synaptic response at the inner hair cell ribbon synapse. J Neurosci 31:7974-7981.

Grant L, Yi E, Glowatzki E (2010) Two modes of release shape the postsynaptic response at the inner hair cell ribbon synapse. J Neurosci 30:4210-4220.

Gray H (1918) Anatomy of the human body, 20th ed. Philadelphia: Lea \& Febiger. Available at: www.bartleby.com/107/ [Accessed February 2, 2013].

Graydon CW, Cho S, Li G-L, Kachar B, Gersdorff H von (2011) Sharp Ca2+ nanodomains beneath the ribbon promote highly synchronous multivesicular release at hair cell synapses. J Neurosci 31:16637-16650.

Gregory FD, Bryan KE, Pangrsic T, Calin-Jageman IE, Moser T, Lee A (2011) Harmonin inhibits presynaptic Cav1.3 Ca2+ channels in mouse inner hair cells. Nat Neurosci 14:1109-1111.

Griesinger CB, Richards CD, Ashmore JF (2002) FM1-43 reveals membrane recycling in adult inner hair cells of the mammalian cochlea. J Neurosci 22:3939 -3952.

Grothe B, Pecka M, McAlpine D (2010) Mechanisms of sound localization in mammals. Physiol Rev 90:983-1012.

Gundelfinger ED, Fejtova A (2012) Molecular organization and plasticity of the cytomatrix at the active zone. Curr Opin Neurobiol 22:423-430.

Hackney CM, Mahendrasingam S, Penn A, Fettiplace R (2005) The concentrations of calcium buffering proteins in mammalian cochlear hair cells. J Neurosci 25:7867-7875. 
Heffner HE, Heffner RS (2008) High-Frequency Hearing. In: The Senses: A Comprehensive Reference (Basbaum AI, Kaneko A, Shepherd GM, Westheimer G, Albright TD, Masland RH, Dallos P, Oertel D, Firestein S, Beauchamp GK, Bushnell MC, Kaas JH, Gardner E, eds), pp 55-60. New York: Academic Press.

Heidelberger R, Heinemann C, Neher E, Matthews G (1994) Calcium dependence of the rate of exocytosis in a synaptic terminal. Nature 371:513-515.

Heil P, Neubauer H (2010) Summing across different active zones can explain the quasi-linear Ca2+-dependencies of exocytosis by receptor cells. Front Syn Neurosci 2:148.

Heinemann C, Chow RH, Neher E, Zucker RS (1994) Kinetics of the secretory response in bovine chromaffin cells following flash photolysis of caged Ca2+. Biophys J 67:25462557.

Hires SA, Shaner NC, Osborne LC, Voglmaier SM (2011) Concurrent imaging of synaptic vesicle recycling and calcium dynamics. Front Mol Neurosci 4:34.

Hires SA, Zhu Y, Tsien RY (2008) Optical measurement of synaptic glutamate spillover and reuptake by linker optimized glutamate-sensitive fluorescent reporters. Proc Natl Acad Sci U S A 105:4411-4416.

Hopsu VK, Arstila AU (1965) An apparent somato-somatic synaptic structure in the pineal gland of the rat. Exp Cell Res 37:484-487.

Huang L-C, Barclay M, Lee K, Peter S, Housley GD, Thorne PR, Montgomery JM (2012) Synaptic profiles during neurite extension, refinement and retraction in the developing cochlea. Neural Dev 7:38.

Huang L-C, Thorne PR, Housley GD, Montgomery JM (2007) Spatiotemporal definition of neurite outgrowth, refinement and retraction in the developing mouse cochlea. Development 134:2925-2933.

Hull C, Studholme K, Yazulla S, Gersdorff H von (2006) Diurnal changes in exocytosis and the number of synaptic ribbons at active zones of an on-type bipolar cell terminal. $\mathbf{J}$ Neurophysiol 96:2025-2033.

Issa NP, Hudspeth AJ (1996) Characterization of fluo-3 labelling of dense bodies at the hair cell's presynaptic active zone. J Neurocytol 25:257-266.

Jackman SL, Choi S-Y, Thoreson WB, Rabl K, Bartoletti TM, Kramer RH (2009) Role of the synaptic ribbon in transmitting the cone light response. Nat Neurosci 12:303-310.

Jarsky T, Tian M, Singer JH (2010) Nanodomain control of exocytosis is responsible for the signaling capability of a retinal ribbon synapse. J Neurosci 30:11885-11895.

Jastrow H, Mach M-A von, Vollrath L (1997) The shape of synaptic ribbons in the rat pineal gland. Cell Tissue Res 287:255-261. 
Jia S, Dallos P, He DZZ (2007) Mechanoelectric transduction of adult inner hair cells. J Neurosci 27:1006-1014.

Johnson CP, Chapman ER (2010) Otoferlin is a calcium sensor that directly regulates SNARE-mediated membrane fusion. J Cell Biol 191:187-197.

Johnson DH (1980) The relationship between spike rate and synchrony in responses of auditory-nerve fibers to single tones. J Acoust Soc Am 68:1115-1122.

Johnson SL, Beurg M, Marcotti W, Fettiplace R (2011) Prestin-driven cochlear amplification is not limited by the outer hair cell membrane time constant. Neuron 70:1143-1154.

Johnson SL, Franz C, Knipper M, Marcotti W (2009) Functional maturation of the exocytotic machinery at gerbil hair cell ribbon synapses. J Physiol 587:1715.

Johnson SL, Franz C, Kuhn S, Furness DN, Rüttiger L, Münkner S, Rivolta MN, Seward EP, Herschman HR, Engel J, Knipper M, Marcotti W (2010) Synaptotagmin IV determines the linear $\mathrm{Ca} 2+$ dependence of vesicle fusion at auditory ribbon synapses. Nat Neurosci 13:45-52.

Johnson SL, Kennedy HJ, Holley MC, Fettiplace R, Marcotti W (2012) The resting transducer current drives spontaneous activity in prehearing mammalian cochlear inner hair cells. J Neurosci 32:10479-10483.

Johnson SL, Marcotti W (2008) Biophysical properties of CaV1.3 calcium channels in gerbil inner hair cells. J Physiol 586:1029-1042.

Johnson SL, Marcotti W, Kros CJ (2005) Increase in efficiency and reduction in Ca2+ dependence of exocytosis during development of mouse inner hair cells. J Physiol 563:177-191.

Juhasz-Vedres G, Gebhart M, Scharinger A, Jangsangthong W, Busquet P, Poggiani C, Sartori S, Mangoni ME, Sinnegger-Brauns MJ, Herzig S, Striessnig J, Koschak A (2011) Functional properties of a newly identified C-terminal splice variant of Cav1.3 L-type Ca2+ channels. J Biol Chem 286:42736-42748.

Kawashima Y, Géléoc GSG, Kurima K, Labay V, Lelli A, Asai Y, Makishima T, Wu DK, Della Santina CC, Holt JR, Griffith AJ (2011) Mechanotransduction in mouse inner ear hair cells requires transmembrane channel-like genes. J Clin Invest 121:4796-4809.

Khimich D, Nouvian R, Pujol R, Tom Dieck S, Egner A, Gundelfinger ED, Moser T (2005) Hair cell synaptic ribbons are essential for synchronous auditory signalling. Nature 434:889-894.

Knipper M, Köpschall I, Rohbock K, Köpke AKE, Bonk I, Zimmermann U, Zenner H-P (1996) Transient expression of NMDA receptors during rearrangement of AMPAreceptor-expressing fibers in the developing inner ear. Cell Tissue Res 287:23-41. 
Kochubey O, Han Y, Schneggenburger R (2009) Developmental regulation of the intracellular $\mathrm{Ca} 2+$ sensitivity of vesicle fusion and $\mathrm{Ca} 2+-$ secretion coupling at the rat calyx of Held. J Physiol 587:3009-3023.

Köppl C (2009) Evolution of sound localisation in land vertebrates. Curr Biol 19:R635-R639.

Kros CJ, Ruppersberg JP, Rüsch A (1998) Expression of a potassium current in inner hair cells during development of hearing in mice. Nature 394:281-284.

Kuriyama H, Jenkins O, Altschuler RA (1994) Immunocytochemical localization of AMPA selective glutamate receptor subunits in the rat cochlea. Hear Res 80:233-240.

Lenzi D, Runyeon JW, Crum J, Ellisman MH, Roberts WM (1999) Synaptic vesicle populations in saccular hair cells reconstructed by electron tomography. $\mathrm{J}$ Neurosci 19:119-132.

Liberman LD, Wang H, Liberman MC (2011) Opposing gradients of ribbon size and AMPA receptor expression underlie sensitivity differences among cochlear-nerve/hair-cell synapses. J Neurosci 31:801-808.

Liberman MC (1978) Auditory-nerve response from cats raised in a low-noise chamber. J Acoust Soc Am 63:442-455.

Liberman MC (1982) Single-neuron labeling in the cat auditory nerve. Science 216:12391241.

Lin K-H, Oleskevich S, Taschenberger H (2011) Presynaptic Ca2+ influx and vesicle exocytosis at the mouse endbulb of Held: a comparison of two auditory nerve terminals. J Physiol 589:4301-4320.

Lindau M, Neher E (1988) Patch-clamp techniques for time-resolved capacitance measurements in single cells. Pflugers Arch 411:137-146.

Loewenstein Y, Kuras A, Rumpel S (2011) Multiplicative dynamics underlie the emergence of the log-normal distribution of spine sizes in the neocortex in vivo. J Neurosci 31:9481-9488.

Logiudice L, Sterling P, Matthews G (2009) Vesicle recycling at ribbon synapses in the finely branched axon terminals of mouse retinal bipolar neurons. Neuroscience 164:15461556.

LoGiudice L, Sterling P, Matthews G (2008) Mobility and turnover of vesicles at the synaptic ribbon. J Neurosci 28:3150-3158.

Lopez-Poveda EA, Meddis R (1996) A physical model of sound diffraction and reflections in the human concha. J Acoust Soc Am 100:3248-3259. 
Lou X, Scheuss V, Schneggenburger R (2005) Allosteric modulation of the presynaptic Ca2+ sensor for vesicle fusion. Nature 435:497-501.

Magupalli VG, Schwarz K, Alpadi K, Natarajan S, Seigel GM, Schmitz F (2008) Multiple RIBEYE-RIBEYE interactions create a dynamic scaffold for the formation of synaptic ribbons. J Neurosci 28:7954-7967.

Maison SF, Parker LL, Young L, Adelman JP, Zuo J, Liberman MC (2007) Overexpression of SK2 channels enhances efferent suppression of cochlear responses without enhancing noise resistance. J Neurophysiol 97:2930-2936.

Marcotti W, Erven A, Johnson SL, Steel KP, Kros CJ (2006) Tmc1 is necessary for normal functional maturation and survival of inner and outer hair cells in the mouse cochlea. $\mathrm{J}$ Physiol 574:677-698.

Marcotti W, Johnson SL, Kros CJ (2004) A transiently expressed SK current sustains and modulates action potential activity in immature mouse inner hair cells. J Physiol 560:691-708.

Marcotti W, Johnson SL, Rusch A, Kros CJ (2003) Sodium and calcium currents shape action potentials in immature mouse inner hair cells. J Physiol (Lond) 552:743-761.

Marvin JS, Borghuis BG, Tian L, Cichon J, Harnett MT, Akerboom J, Gordus A, Renninger SL, Chen T-W, Bargmann CI, Orger MB, Schreiter ER, Demb JB, Gan W-B, Hires SA, Looger LL (2013) An optimized fluorescent probe for visualizing glutamate neurotransmission. Nat Methods 10:162-170.

Matthews G, Fuchs P (2010) The diverse roles of ribbon synapses in sensory neurotransmission. Nat Rev Neurosci 11:812-822.

Matthews G, Sterling P (2008) Evidence that vesicles undergo compound fusion on the synaptic ribbon. J Neurosci 28:5403-5411.

Matveev V, Bertram R, Sherman A (2011) Calcium cooperativity of exocytosis as a measure of Ca2+ channel domain overlap. Brain Res 1398:126-138.

McLean WJ, Smith KA, Glowatzki E, Pyott SJ (2009) Distribution of the Na,K-ATPase $\alpha$ subunit in the rat spiral ganglion and organ of Corti. J Assoc Res Otolaryngol 10:3749.

Mehta B, Snellman J, Chen S, Li W, Zenisek D (2013) Synaptic ribbons influence the size and frequency of miniature-like evoked postsynaptic currents. Neuron 77:516-527.

Merchan-Perez A, Liberman MC (1996) Ultrastructural differences among afferent synapses on cochlear hair cells: correlations with spontaneous discharge rate. J Comp Neurol 371:208-221. 
Meyer AC, Frank T, Khimich D, Hoch G, Riedel D, Chapochnikov NM, Yarin YM, Harke B, Hell SW, Egner A, Moser T (2009) Tuning of synapse number, structure and function in the cochlea. Nat Neurosci 12:444-453.

Meyer AC, Moser T (2010) Structure and function of cochlear afferent innervation. Curr Opin Otolaryngol Head Neck Surg 18:441-446.

Meyer AC, Neher E, Schneggenburger R (2001) Estimation of quantal size and number of functional active zones at the calyx of Held synapse by nonstationary EPSC variance analysis. J Neurosci 21:7889-7900.

Miesenböck G, De Angelis DA, Rothman JE (1998) Visualizing secretion and synaptic transmission with $\mathrm{pH}$-sensitive green fluorescent proteins. Nature 394:192-195.

Mikaelian D, Ruben RJ (1965) Development of hearing in the normal CBA-J mouse: Correlation of physiological observations with behavioral responses and with cochlear anatomy. Acta Otolaryngol 59:451-461.

Mikaelian DO (1979) Development and degeneration of hearing in the c57/b16 mouse: Relation of electrophysiologic responses from the round window and cochlear nucleus to cochlear anatomy and behavioral responses. Laryngoscope 89:1-15.

Moser T, Beutner D (2000) Kinetics of exocytosis and endocytosis at the cochlear inner hair cell afferent synapse of the mouse. Proc Natl Acad Sci USA 97:883-888.

Mueller F, Morisaki T, Mazza D, McNally JG (2012) Minimizing the impact of photoswitching of fluorescent proteins on FRAP analysis. Biophys J 102:1656-1665.

Naraghi M (1997) T-jump study of calcium binding kinetics of calcium chelators. Cell Calcium 22:255-268.

Naraghi M, Neher E (1997) Linearized buffered Ca2+ diffusion in microdomains and its implications for calculation of $[\mathrm{Ca} 2+]$ at the mouth of a calcium channel. J Neurosci 17:6961.

Neef A, Khimich D, Pirih P, Riedel D, Wolf F, Moser T (2007) Probing the mechanism of exocytosis at the hair cell ribbon synapse. J Neurosci 27:12933-12944.

Neef J, Gehrt A, Bulankina AV, Meyer AC, Riedel D, Gregg RG, Strenzke N, Moser T (2009) The $\mathrm{Ca} 2+$ channel subunit $\beta 2$ regulates $\mathrm{Ca} 2+$ channel abundance and function in inner hair cells and is required for hearing. J Neurosci 29:10730.

Nemzou RM, Bulankina AV, Khimich D, Giese A, Moser T (2006) Synaptic organization in cochlear inner hair cells deficient for the CaV1.3 ( $\alpha 1 \mathrm{D})$ subunit of L-type Ca2+ channels. Neuroscience 141:1849-1860.

Nishikawa S, Sasaki F (1996) Internalization of styryl dye FM1-43 in the hair cells of lateral line organs in Xenopus larvae. J Histochem Cytochem 44:733-741. 
Nouvian R (2007) Temperature enhances exocytosis efficiency at the mouse inner hair cell ribbon synapse. J Physiol 584:535-542.

Nouvian R, Neef J, Bulankina AV, Reisinger E, Pangršič T, Frank T, Sikorra S, Brose N, Binz T, Moser T (2011) Exocytosis at the hair cell ribbon synapse apparently operates without neuronal SNARE proteins. Nat Neurosci 14:411-413.

Ohlemiller KK, Echteler SM (1990) Functional correlates of characteristic frequency in single cochlear nerve fibers of the Mongolian gerbil. J Comp Physiol A 167:329-338.

Okumoto S, Looger LL, Micheva KD, Reimer RJ, Smith SJ, Frommer WB (2005) Detection of glutamate release from neurons by genetically encoded surface-displayed FRET nanosensors. Proc Natl Acad Sci U S A 102:8740-8745.

Oliver D, Knipper M, Derst C, Fakler B (2003) Resting potential and submembrane calcium concentration of inner hair cells in the isolated mouse cochlea are set by KCNQ-type potassium channels. J Neurosci 23:2141-2149.

Palmer AR, Russell IJ (1986) Phase-locking in the cochlear nerve of the guinea-pig and its relation to the receptor potential of inner hair-cells. Hear Res 24:1-15.

Pangršič T, Lasarow L, Reuter K, Takago H, Schwander M, Riedel D, Frank T, Tarantino LM, Bailey JS, Strenzke N, Brose N, Muller U, Reisinger E, Moser T (2010) Hearing requires otoferlin-dependent efficient replenishment of synaptic vesicles in hair cells. Nat Neurosci 13:869-876.

Pangršič T, Reisinger E, Moser T (2012) Otoferlin: a multi-C2 domain protein essential for hearing. Trends Neurosci 35:671-680.

Parsons TD, Lenzi D, Almers W, Roberts WM (1994) Calcium-triggered exocytosis and endocytosis in an isolated presynaptic cell: Capacitance measurements in saccular hair cells. Neuron 13:875-883.

Parsons TD, Sterling P (2003) Synaptic Ribbon. Conveyor belt or safety belt? Neuron 37:379-382.

Platzer J, Engel J, Schrott-Fischer A, Stephan K, Bova S, Chen H, Zheng H, Striessnig J (2000) Congenital deafness and sinoatrial node dysfunction in mice lacking class D Ltype Ca2+ channels. Cell 102:89-97.

Press WH, Flannery BP, Teukolsky SA, Vetterling WT (1992) Numerical recipes in C: The art of scientific computing, 2nd ed. New York, NY, USA: Cambridge University Press.

Puel J-L, Ruel J, Guitton M, Pujol R (2002) The inner hair cell afferent/efferent synapses revisited: a basis for new therapeutic strategies. Adv Otorhinolaryngol 59:124-130.

Purves D (2004) Neuroscience, 3rd ed. Sunderland, Mass: Sinauer Associates, Publishers. 
Raouf R, Rugiero F, Kiesewetter H, Hatch R, Hummler E, Nassar MA, Wang F, Wood JN (2012) Sodium channels and mammalian sensory mechanotransduction. Mol Pain 8:21.

Regus-Leidig H, Tom Dieck S, Specht D, Meyer L, Brandstätter JH (2009) Early steps in the assembly of photoreceptor ribbon synapses in the mouse retina: the involvement of precursor spheres. J Comp Neurol 512:814-824.

Reichenbach T, Hudspeth AJ (2012) Discrimination of low-frequency tones employs temporal fine structure. PLoS ONE 7:e45579.

Reisinger E, Bresee C, Neef J, Nair R, Reuter K, Bulankina A, Nouvian R, Koch M, Buckers J, Kastrup L, Roux I, Petit C, Hell SW, Brose N, Rhee J-S, Kugler S, Brigande JV, Moser T (2011) Probing the functional equivalence of otoferlin and synaptotagmin 1 in exocytosis. J Neurosci 31:4886-4895.

Roberts WM, Jacobs RA, Hudspeth AJ (1990) Colocalization of ion channels involved in frequency selectivity and synaptic transmission at presynaptic active zones of hair cells. J Neurosci 10:3664-3684.

Rose JE, Brugge JF, Anderson DJ, Hind JE (1967) Phase-locked response to low-frequency tones in single auditory nerve fibers of the squirrel monkey. J Neurophysiol 30:769793.

Roux I, Hosie S, Johnson SL, Bahloul A, Cayet N, Nouaille S, Kros CJ, Petit C, Safieddine S (2009) Myosin VI is required for the proper maturation and function of inner hair cell ribbon synapses. Hum Mol Genet 18:4615-4628.

Roux I, Safieddine S, Nouvian R, Grati M, Simmler M-C, Bahloul A, Perfettini I, Le Gall M, Rostaing P, Hamard G, Triller A, Avan P, Moser T, Petit C (2006) Otoferlin, defective in a human deafness form, is essential for exocytosis at the auditory ribbon synapse. Cell 127:277-289.

Ruel J, Emery S, Nouvian R, Bersot T, Amilhon B, Rybroek JMV, Rebillard G, Lenoir M, Eybalin M, Delprat B, Sivakumaran TA, Giros B, Mestikawy SE, Moser T, Smith RJH, Lesperance MM, Puel J-L (2008) Impairment of SLC17A8 encoding vesicular glutamate transporter-3, VGLUT3, underlies nonsyndromic deafness DFNA25 and inner hair cell dysfunction in null mice. Am J Hum Genet 83:278-292.

Ruel J, Nouvian R, Gervais d'Aldin C, Pujol R, Eybalin M, Puel JL (2001) Dopamine inhibition of auditory nerve activity in the adult mammalian cochlea. Eur J Neurosci 14:977-986.

Russell IJ, Kössl M, Richardson GP (1992) Nonlinear mechanical responses of mouse cochlear hair bundles. Proc Biol Sci 250:217-227.

Rutherford MA, Chapochnikov NM, Moser T (2012) Spike encoding of neurotransmitter release timing by spiral ganglion neurons of the cochlea. J Neurosci 32:4773-4789. 
Sachs MB, Winslow RL, Sokolowski BHA (1989) A computational model for rate-level functions from cat auditory-nerve fibers. Hear Res 41:61-69.

Safieddine S, Bartolami S, Wenthold RJ, Eybalin M (1996) Pre- and postsynaptic M3 muscarinic receptor mRNAs in the rodent peripheral auditory system. Brain Res Mol Brain Res 40:127-135.

Safieddine S, Eybalin M (1992) Triple immunofluorescence evidence for the coexistence of acetylcholine, enkephalins and calcitonin gene-related peptide within efferent (olivocochlear) neurons of rats and guinea-pigs. Eur J Neurosci 4:981-992.

Safieddine S, Wenthold RJ (1999) SNARE complex at the ribbon synapses of cochlear hair cells: analysis of synaptic vesicle- and synaptic membrane-associated proteins. Eur $\mathbf{J}$ Neurosci 11:803-812.

Sankaranarayanan S, De Angelis D, Rothman JE, Ryan TA (2000) The use of pHluorins for optical measurements of presynaptic activity. Biophys J 79:2199-2208.

Sätzler K, Söhl LF, Bollmann JH, Borst JGG, Frotscher M, Sakmann B, Lübke JHR (2002) Three-dimensional reconstruction of a calyx of Held and its postsynaptic principal neuron in the medial nucleus of the trapezoid body. J Neurosci 22:10567-10579.

Schmitz F, Königstorfer A, Südhof TC (2000) RIBEYE, a component of synaptic ribbons: A protein's journey through evolution provides insight into synaptic ribbon function. Neuron 28:857-872.

Schneggenburger R, Neher E (2000) Intracellular calcium dependence of transmitter release rates at a fast central synapse. Nature 406:889-893.

Schneider CA, Rasband WS, Eliceiri KW (2012) NIH Image to ImageJ: 25 years of image analysis. Nat Methods 9:671-675.

Schug N, Braig C, Zimmermann U, Engel J, Winter H, Ruth P, Blin N, Pfister M, Kalbacher H, Knipper M (2006) Differential expression of otoferlin in brain, vestibular system, immature and mature cochlea of the rat. Eur J Neurosci 24:3372-3380.

Seal RP, Akil O, Yi E, Weber CM, Grant L, Yoo J, Clause A, Kandler K, Noebels JL, Glowatzki E, Lustig LR, Edwards RH (2008) Sensorineural deafness and seizures in mice lacking vesicular glutamate transporter 3. Neuron 57:263-275.

Sendin G, Bulankina AV, Riedel D, Moser T (2007) Maturation of ribbon synapses in hair cells is driven by thyroid hormone. J Neurosci 27:3163 -3173.

Sheets L, Kindt KS, Nicolson T (2012) Presynaptic CaV1.3 channels regulate synaptic ribbon size and are required for synaptic maintenance in sensory hair cells. J Neurosci 32:17273-17286. 
Sheets L, Trapani JG, Mo W, Obholzer N, Nicolson T (2011) Ribeye is required for presynaptic $\mathrm{CaV} 1.3 \mathrm{a}$ channel localization and afferent innervation of sensory hair cells. Development 138:1309-1319.

Sheng J, He L, Zheng H, Xue L, Luo F, Shin W, Sun T, Kuner T, Yue DT, Wu L-G (2012) Calcium-channel number critically influences synaptic strength and plasticity at the active zone. Nat Neurosci 15:998-1006.

Si F, Brodie H, Gillespie PG, Vazquez AE, Yamoah EN (2003) Developmental assembly of transduction apparatus in chick basilar papilla. J Neurosci 23:10815-10826.

Siegel JH, Brownell WE (1986) Synaptic and Golgi membrane recycling in cochlear hair cells. J Neurocytol 15:311-328.

Sinnecker D, Voigt P, Hellwig N, Schaefer M (2005) Reversible photobleaching of enhanced green fluorescent proteins. Biochemistry 44:7085-7094.

Snellman J, Mehta B, Babai N, Bartoletti TM, Akmentin W, Francis A, Matthews G, Thoreson W, Zenisek D (2011) Acute destruction of the synaptic ribbon reveals a role for the ribbon in vesicle priming. Nat Neurosci 14:1135-1141.

Sobkowicz H, Rose J, Scott G, Slapnick S (1982) Ribbon synapses in the developing intact and cultured organ of Corti in the mouse. J Neurosci 2:942-957.

Sonntag M, Englitz B, Kopp-Scheinpflug C, Rübsamen R (2009) Early postnatal development of spontaneous and acoustically evoked discharge activity of principal cells of the medial nucleus of the trapezoid body: An in vivo study in mice. J Neurosci 29:9510 9520 .

Südhof TC (2001) Synaptotagmins: Why so many? J Biol Chem 277:7629-7632.

Sun J, Pang ZP, Qin D, Fahim AT, Adachi R, Südhof TC (2007) A dual-Ca2+-sensor model for neurotransmitter release in a central synapse. Nature 450:676-682.

Taberner AM, Liberman MC (2005) Response properties of single auditory nerve fibers in the mouse. J Neurophysiol 93:557-569.

Tan BZ, Jiang F, Tan MY, Yu D, Huang H, Shen Y, Soong TW (2011) Functional characterization of alternative splicing in the C-terminus of L-type CaV1.3 channels. $\mathrm{J}$ Biol Chem 286:42725-42735.

Thoreson WB, Tranchina D, Witkovsky P (2003) Kinetics of synaptic transfer from rods and cones to horizontal cells in the salamander retina. Neuroscience 122:785-798.

Tritsch NX, Rodríguez-Contreras A, Crins TTH, Wang HC, Borst JGG, Bergles DE (2010) Calcium action potentials in hair cells pattern auditory neuron activity before hearing onset. Nat Neurosci 13:1050-1052. 
Tritsch NX, Yi E, Gale JE, Glowatzki E, Bergles DE (2007) The origin of spontaneous activity in the developing auditory system. Nature 450:50-55.

Voglmaier SM, Kam K, Yang H, Fortin DL, Hua Z, Nicoll RA, Edwards RH (2006) Distinct endocytic pathways control the rate and extent of synaptic vesicle protein recycling. Neuron 51:71-84.

Von Gersdorff H, Vardi E, Matthews G, Sterling P (1996) Evidence that vesicles on the synaptic ribbon of retinal bipolar neurons can be rapidly released. Neuron 16:12211227.

Walsh EJ, McGee J (1987) Postnatal development of auditory nerve and cochlear nucleus neuronal responses in kittens. Hear Res 28:97-116.

Wang L-Y, Neher E, Taschenberger H (2008) Synaptic vesicles in mature calyx of held synapses sense higher nanodomain calcium concentrations during action potentialevoked glutamate release. J Neurosci 28:14450-14458.

Winter IM, Robertson D, Yates GK (1990) Diversity of characteristic frequency rate-intensity functions in guinea pig auditory nerve fibres. Hear Res 45:191-202.

Witkovsky P, Schmitz Y, Akopian A, Krizaj D, Tranchina D (1997) Gain of rod to horizontal cell synaptic transfer: relation to glutamate release and a dihydropyridine-sensitive calcium current. J Neurosci 17:7297-7306.

Wu MM, Llopis J, Adams S, McCaffery JM, Kulomaa MS, Machen TE, Moore H-PH, Tsien RY (2000) Organelle pH studies using targeted avidin and fluorescein-biotin. Chem Biol 7:197-209.

Yang PS, Alseikhan BA, Hiel H, Grant L, Mori MX, Yang W, Fuchs PA, Yue DT (2006) Switching of $\mathrm{Ca} 2+-$ dependent inactivation of $\mathrm{CaV} 1.3$ channels by calcium binding proteins of auditory hair cells. J Neurosci 26:10677 -10689.

Yang Y-M, Fedchyshyn MJ, Grande G, Aitoubah J, Tsang CW, Xie H, Ackerley CA, Trimble WS, Wang L-Y (2010) Septins regulate developmental switching from microdomain to nanodomain coupling of $\mathrm{Ca} 2+$ influx to neurotransmitter release at a central synapse. Neuron 67:100-115.

Yates GK, Winter IM, Robertson D (1990) Basilar membrane nonlinearity determines auditory nerve rate-intensity functions and cochlear dynamic range. Hear Res 45:203219.

Zampini V, Johnson SL, Franz C, Lawrence ND, Muenkner S, Engel J, Knipper M, Magistretti J, Masetto S, Marcotti W (2010) Elementary properties of CaV1.3 Ca2+ channels expressed in mouse cochlear inner hair cells. J Physiol 588:187-199.

Zenisek D, Horst NK, Merrifield C, Sterling P, Matthews G (2004) Visualizing synaptic ribbons in the living cell. J Neurosci 24:9752-9759. 
Zheng J, Shen W, He DZZ, Long KB, Madison LD, Dallos P (2000) Prestin is the motor protein of cochlear outer hair cells. Nature 405:149-155.

Zhu Y, Xu J, Heinemann SF (2009) Two pathways of synaptic vesicle retrieval revealed by single-vesicle imaging. Neuron 61:397-411. 


\section{Acknowledgements}

I would like to thank my supervisor Tobias Moser for the opportunity to work in his lab and on this project. I am grateful of his continuous support, helpful scientific discussions, the ample supply of ideas, and his constant encouragement for scientific exposure and interactions.

I would also like to thank other members of my thesis committee, Erwin Neher and Nils Brose, for all their critical comments and suggestions during the entire project.

I am greatly indebted to Thomas Frank for his introduction and subsequent advices to electrophysiology and imaging techniques, for establishment of the confocal imaging setup, and for many helpful discussions.

I am thankful to Kirsten Reuter and Christiane Senger-Freitag for painstakingly injecting embryos for the vglut1-pHluorin project, Kirsten again for constructing the expression vector, and Sebastian Kügler for producing the virus. I would also like to thank Sonja Wojcik and Cordelia Imig for advice and help on molecular biology.

I thank Mark A. Rutherford for the Cav1.3 immunohistochemistry, Carolin Wichmann for performing electron microscopy. I thank Tina Pangršič for teaching me and collaborating in the $\mathrm{Ca}^{2+}$ uncaging experiments, countless candid and in-depth comments, fun discussions and having a hard-to-pronounce-and-type name.

A lot of thanks go to Gerhard Hoch, Christiane Senger-Freitag, Sandra Gerke and Nina Dankenbrink-Werder for their professional technical support.

I thank Jakob Neef for selfless troubleshooting and aid in the lab, and for knowing things that nobody else in the lab knows. I will thank Tzu-Lun Wang, Alejandro MendozaSchultz and Nikolai Chapochnikov for scientific and non-scientific dialogues. I am grateful of all the members of the InnerEarLab that I could not enumerate here for providing a positive, friendly and enjoyable environment for both work and life in all these years.

I greatly appreciate the work of Michael Hörner and Sandra Drube of the Neurosciences Coordination Office, which made my adaptation to Germany and everything else smooth and simple.

I would also like to acknowledge the financial support by the state of Lower Saxony in the form of a Georg-Christoph-Lichtenberg stipend, by Göttingen Graduate School for Neurosciences, Biophysics, and Molecular Biosciences through its Bridging Fund, and by the Croucher Foundation through an honorary scholarship.

Finally and most importantly, I would like to thank my parents for their unfailing support, understanding and tolerance, and all family members for their encouragement. Without their backup, I would not be able to pursue my studies so far away from home, relatively care-free. Many thanks for tolerating my selfish absence at times. 